International Journal of Pure and Applied Mathematics

Volume 102 No. 2 2015, 301-347

ISSN: 1311-8080 (printed version); ISSN: 1314-3395 (on-line version)

url: http://www.ijpam.eu

doi: http://dx.doi.org/10.12732/ijpam.v102i2.11

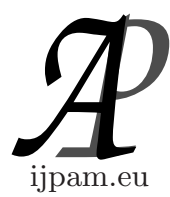

\title{
ON THE SEGRE UPPER BOUND OF THE REGULARITY FOR FAT POINTS IN $\mathbb{P}^{4}$, II
}

\author{
E. Ballico \\ Department of Mathematics \\ University of Trento \\ 38123 Povo (Trento) - Via Sommarive, 14, ITALY
}

\begin{abstract}
We study the generalized Segre bound in $\mathbb{P}^{4}$ for fat points schemes. In this second part we prove the quartic case and an inductive lemma. We also discuss stronger vanishing theorems for sets with additional properties.
\end{abstract}

AMS Subject Classification: 13A02, 13D02, 14N05

Key Words: fat point, index of regularity, homogeneous ideal, 4-dimensional projective space

\section{Introduction}

Fix a finite set $S \subset \mathbb{P}^{4}, S \neq \emptyset$. For each $P \in S$ fix an integer $m_{P}>0$ and set $Z:=\sum_{P \in S} m_{P} P$. Set $s:=\sharp(S)$ and let $m_{1} \geq \cdots \geq m_{s}$ be the multiplicities of the points of $Z$.

We recall that in $\mathbb{P}^{4}$ the Segre conjecture (or the generalized Segre conjecture) may be restated in the following way: $h^{1}\left(\mathcal{I}_{Z}(d)\right)=0$ if $m_{1} \leq d$ and $w(L) \leq c d+1$ for every $c$-dimensional linear subspace $L \subseteq \mathbb{P}^{4}, c=1,2,3,4$. We recall that this conjecture is true in $\mathbb{P}^{2}([4],[8])$ in $\mathbb{P}^{3}([7],[9])$, if $S$ is in linearly

Received: April 18, 2015

(c) 2015 Academic Publications, Ltd. url: www.acadpubl.eu 
general position ([5]) and if $\sharp(S)$ is small ([3], [12], [2]). We fix an integer $d>0$ and we take $Z$ satisfying the Segre condition in degree $d$. The aim (not yet reached) of our series of papers (of which this is the second part) is to prove that $h^{1}\left(\mathcal{I}_{Z}(d)\right)=0$. This was proved if $d=3$ in [1]. In this paper we make a part of an inductive steps $d-2, d-1 \Longrightarrow d$ (we assume the full conjecture for degrees $<d$, but we prove that $h^{1}\left(\mathcal{I}_{Z}(d)\right)=0$ only if $Z$ satisfies another numerical condition, i.e. we prove the following result.

Lemma 1. Fix an integer $d \geq 4$ and assume that the Segre condition is true in degrees $d-2$ and $d-1$, hat $Z$ satisfies the Segre conditions in degree $d$ and that $m_{1}+m_{2} \leq d$. Then $h^{1}\left(\mathcal{I}_{Z}(d)\right)=0$.

We also prove the case $d=4$ (Proposition 2) and a critical case for $d=5$ (Proposition 3). See Section 2 for the introduction of notation needed in the third and fourth parts of this series. We introduce it to point out why some steps of the proof of Lemma 1 work in other cases and avoid to repeat them in the other parts.

We also prove the following result for an arbitrary $\mathbb{P}^{n}$ when $S$ satisfies a strong condition.

Proposition 1. Assume the existence of an integer $x$ such that $2 \leq x<d$ and for any $S^{\prime} \subseteq S$ we have $h^{0}\left(\mathcal{I}_{S^{\prime}}(x)\right)=\max \left\{0,\left(\begin{array}{c}n+x \\ n\end{array}\right)-\sharp\left(S^{\prime}\right)\right\}$. Set $r:=$ $-1+\min \left\{\left(\begin{array}{c}n+x \\ n\end{array}\right), \sharp(S)\right\}$. Assume $m_{1}+m_{2} \leq d+1$ and $m_{1}+\cdots+m_{s} \leq r\lfloor d / x\rfloor+1$. Then $h^{1}\left(\mathcal{I}_{Z}(d)\right)=0$.

The condition for $S$ is weaker than the Uniform Position Property used in [11] (it does not even implies that $S$ is in linearly general position), but the proof of Proposition 1 is just a reduction to the linearly general position case proved in [5, Theorem 1.4].

\section{Preliminaries}

We fix an integer $d>0$ and assume that the generalized Segre conjecture is true in $\mathbb{P}^{4}$ for all positive integers $<d$. We fix $Z=\sum_{p \in S} m_{p} p$ and assume that $Z$ satisfies the assumptions of the generalized Segre conjecture in degree $d$, i.e. we assume $d \geq m_{1}, w\left(\mathbb{P}^{4}\right) \leq 4 d+1$ and $w(E) \leq i d+i-2$ for $i=1,2,3$ and each $i$-dimensional linear subspace $E \subset \mathbb{P}^{4}$. We use induction on the integer $w\left(\mathbb{P}^{4}\right)=\sum_{p \in S} m_{p}$. In particular we assume $h^{1}\left(\mathcal{I}_{W}(d)\right)=0$ if $W=\sum_{p \in S^{\prime}} m_{p} p$, where $S^{\prime} \subsetneq S$. For each $p \in \mathbb{P}^{4}$ let $m_{p}$ denote the multiplicity of $p$ in $Z$. Hence $m_{p}=0$ if and only if $p \notin S$. We say that $Z$ satisfies $\diamond$ if $m_{1}+m_{2} \geq d+1$. We assume that $\diamond$ fails in sections $3,4,5,6,7$. We say that $Z$ satisfies 
$m_{1}>m / 2+1$. We say that $Z$ satisfies $\diamond \diamond$ if $d$ is odd and $m_{1}=m_{2}=(d+1) / 2$.

Notation 1. For any set $A \subseteq \mathbb{P}^{4}$ set $w(A):=\sum_{P \in S \cap A} m_{P}$. Write $w_{Z}(A):=w(A)$. If $U \subset \mathbb{P}^{4}$ is any finite subset and for each $P \in U$ we take a nonnegative integer $n_{P}$, then set $w_{W}(A):=\sum_{P \in U \cap A} n_{P}$, where $W:=\sum_{P \in U} n_{P} P$. Let $B_{1}$ (resp. $\left.B_{1}^{\prime}\right)$ be the set of all lines $L \subset \mathbb{P}^{4}$ such that $w(L)=d+1$ (resp. $w(L)=d$ ). Let $B_{2}$ (resp. $B_{2}^{\prime}$ ) be the set of all planes $L \subset \mathbb{P}^{4}$ such that $2 d \leq w(L) \leq 2 d+1$ (resp. $2 d-2 \leq w(L) \leq 2 d-1$ ). Let $B_{3}$ (resp. $B_{3}^{\prime}$ ) be the set of all hyperplanes $L \subset \mathbb{P}^{4}$ such that $3 d-1 \leq w(L) \leq 3 d+1$ (resp. $3 d-4 \leq w(L) \leq 3 d-2)$.

Proof. Since $U \in B_{3}$, we have $\sharp(U \cap S) \geq 4$ and hence $\mathbb{P}^{4}$ satisfies the Segre condition for $\mathbb{P}^{4}$. Since $w\left(\mathbb{P}^{4} \backslash U\right) \leq d$, $\operatorname{Res}_{U}(Z)$ satisfies the Segre condition with respect to all lines, all planes $A$ with $w(A)=2 d$ and all hyperplanes with $w(A)=3 d-1$. Fix a plane $A$ such that $w(A)=2 d+1$. Since $m_{1} \leq d$, we have $\sharp(A \cap H \cap S) \geq 2$. Fix a hyperplane $G$ with $w(G) \geq 3 d$. Since $m_{1}+m_{2} \leq d+1$ and $w\left(\mathbb{P}^{4} \backslash U\right)+d+1<3 d$, we get $\sharp(G \cap U \cap S) \geq 3$.

For any set $E \subseteq \mathbb{P}^{4}$ let $\langle E\rangle$ denote the linear span of $E$, i.e. the minimal linear subspace of $\mathbb{P}^{4}$ containing $E$. We use the following lemmas ([1], Lemmas 3 and 1).

Lemma 2. If there is a hyperplane $H \subset \mathbb{P}^{4}$ such that $\sharp(S \cap H) \geq \sharp(S)-1$, then $h^{1}\left(\mathcal{I}_{Z}(d)\right)=0$.

Lemma 3. If $m_{1}=d$, then $h^{1}\left(\mathcal{I}_{Z}(d)\right)=0$.

By Lemma 3 we may assume $m_{1} \leq d-1$.

Proof of Proposition 1: We will even prove that $h^{1}\left(\mathcal{I}_{Z}(x\lfloor d / x\rfloor)\right)=0$. Let $\nu_{x}: \mathbb{P}^{n} \rightarrow \mathbb{P}^{N}, N:=\left(\begin{array}{c}n+x \\ n\end{array}\right)-1$, the Veronese embedding of $\mathbb{P}^{n}$ by the projective space of all degree $x$ forms in $n$ variable. If $h^{1}\left(\mathcal{I}_{\nu_{x}(Z)}(\lfloor d / x\rfloor)\right)=0$, then $h^{1}\left(\mathcal{I}_{Z}(x\lfloor d / x\rfloor)\right)=0$. Let $W \subset \mathbb{P}^{N}$ be the fat point scheme of $\mathbb{P}^{N}$ with support $\nu_{x}(S)$ and multiplicity $m_{p}$ at the point $\nu_{x}(p), p \in S$. Since $W$ is zerodimension, $h^{1}\left(W, \mathcal{I}_{Z, W}(d)\right)=0$ and so the restriction map $H^{0}\left(\mathcal{O}_{W}(\lfloor d / x\rfloor)\right) \rightarrow$ $H^{0}\left(\mathcal{O}_{\nu_{x}(Z)}(\lfloor d / x\rfloor)\right)$ is surjective. Hence if

$$
h^{1}\left(\mathbb{P}^{N}, \mathcal{I}_{W}(\lfloor d / x\rfloor)\right)=0,
$$

then $h^{1}\left(\mathcal{I}_{\nu_{x}(Z)}(\lfloor d / x\rfloor)\right)=0$. The linear span $M:=\left\langle\nu_{x}(S)\right\rangle$ has dimension $r$. By the case $S \subset H$ of Lemma 2 applied $N-\operatorname{dim}(N)$ times to $\mathbb{P}^{N}$ it is sufficient to prove that $h^{1}\left(M, \mathcal{I}_{W \cap M}(\lfloor d / x\rfloor)\right)=0$. This is true by [5, Theorem 1.4], because $\nu_{x}(S)$ is by assumption in linearly general position in $M$. 
For any hypersurface $T \subset \mathbb{P}^{4}$ and any scheme $A \subset \mathbb{P}^{4}$ the residual scheme $\operatorname{Res}_{T}(A)$ of $A$ with respect to $T$ is the closed subscheme of $\mathbb{P}^{4}$ with $\mathcal{I}_{A}: \mathcal{I}_{T}$ as its ideal scheme. Set $a:=\operatorname{deg}(T)$. We have an exact sequence (called the residual exact sequence of $T$ ):

$$
0 \rightarrow \mathcal{I}_{\operatorname{Res}_{T}(A)}(t-a) \rightarrow \mathcal{I}_{A}(t) \rightarrow \mathcal{I}_{T \cap A, T}(t) \rightarrow 0
$$

Remark 1. By (1) to prove that $h^{1}\left(\mathcal{I}_{Z}(d)\right)=0$ it is sufficient to find $T$ such that $h^{1}\left(T, \mathcal{I}_{T \cap Z, T}(d)\right)=0$ and $h^{1}\left(\mathcal{I}_{\operatorname{Res}_{T}(Z)}(t-a)\right)=0$. We will use the cases $a=1,2$. In the case of hyperplanes we always have $h^{1}\left(T, \mathcal{I}_{Z \cap T, T}(d)\right)=0$ $([9],[7])$. Fix a hyperplane $H \subset \mathbb{P}^{4}$ and a quadric $Q \subset \mathbb{P}^{4}$. To check that $\operatorname{Res}_{H}(Z)$ satisfies the Segre conditions in degree $d-1$ it is sufficient to check it for $\mathbb{P}^{4}$ (where it is true if $\sharp(S \cap H) \geq 4$, for all $B \in B_{1}$ (we only need to prove that $B \cap H \cap S \neq \emptyset$ ), for all $A \in B_{2}$ (we only need to prove that $\sharp(A \cap H \cap S) \geq w(A)-2 d-1$ ) and for all $U \in B_{3}$ (we only need to prove that $\sharp(U \cap H \cap S) \geq w(U)-3 d-4)$. To check that $\operatorname{Res}_{Q}(Z)$ satisfies the Segre conditions in degree $d-1$ it is sufficient to check it for $\mathbb{P}^{4}$ (it is true if $\sharp(S \cap Q) \geq 8)$, for all lines, all planes and all hyperplanes. To check it for all lines it is sufficient to check it for the elements $B \in B_{1} \cup B_{1}^{\prime}$; if $B \in B_{1}^{\prime}$ it is sufficient to have $B \cap S \cap Q \neq \emptyset$, if $B \in B_{1}$ it is sufficient to have $\sharp(B \cap S \cap Q) \geq 2$. To check it for all planes it is sufficient to test if for all $A \in B_{2} \cup B_{2}^{\prime}$; if $A \in B_{2} \cup B_{2}^{\prime}$ it is sufficient to check that $\sharp(S \cap Q) \geq w(A)-2 d+3$. To check it for all hyperplanes it is sufficient to prove that $\sharp(U \cap S \cap Q) \geq w(U)-3 d-7$ for all $U \in B_{3} \cup B_{3}^{\prime}$. If $S \cap Q \neq \emptyset$, then the inductive assumption on the weight gives $h^{1}\left(\mathcal{I}_{\sum_{p \in S \cap Q} m_{p} p}(d)\right)=0$; since $Q \cap\left(\sum_{p \in S \cap Q} m_{p} p\right)=Q \cap Z$, we get $h^{1}\left(\mathcal{I}_{Z \cap Q}(d)\right)=0$ and hence $h^{1}\left(Q, \mathcal{I}_{Z \cap Q, Q}(d)\right)=0$.

We use the following remarks $([1])$.

Remark 2. If $A \in B_{i} \cup B_{i}^{\prime}$, then $A \cap S$ spans $A$ (use the Segre conditions for the proper linear subspaces of $A$, for $B_{1}^{\prime}$ we also need the assumption $m_{1} \leq$ $d-1)$.

Remark 3. Tale $L, R \in B_{1}$ such that $L \cap R \neq \emptyset$. Since $w(E) \leq 2 d+1$ and $w(L)=w(R)=d+1$, we have $L \cap R \cap S \neq \emptyset$.

Remark 4. Fix an integer $t>0$. Let $A \subset \mathbb{P}^{4}$ be a zero-dimensional scheme such that $h^{1}\left(\mathcal{I}_{A}(t)\right)=0$. Let $B$ be a subscheme of $A$. Since the restriction map $H^{0}\left(\mathcal{O}_{A}(t)\right) \rightarrow H^{0}\left(\mathcal{O}_{B}(t)\right)$ is surjective we have $h^{1}\left(\mathcal{I}_{B}(t)\right)=0$. In particular for every hypersurface $T \subset \mathbb{P}^{4}$ we have $h^{1}\left(\mathcal{I}_{A \cap T}(t)\right)=0$. Hence $h^{1}\left(T, \mathcal{I}_{T \cap A}(t)\right)=0$.

Remark 5. Assume the existence of $L_{i} \in B_{1}, i=1,2,3$, such that $L_{i} \cap L_{j}=\emptyset$ for all $i \neq j$. Let $L \subset \mathbb{P}^{4}$ be a line with $w(L) \geq d-1$. Since 
$w\left(\mathbb{P}^{4}\right) \leq 4 d+1$, then $S \cap L \cap\left(L_{1} \cup L_{2} \cup L_{3}\right) \neq \emptyset$.

Remark 6. We have $m_{1}+m_{2} \leq d$, i.e. not $\diamond$, if and only if $\sharp(B \cap S) \geq 3$ for all $B \in B_{1}$. Assume $m_{1}+m_{2} \leq d$. We have $\sharp(B \cap S) \geq 3$ for all $B \in B_{1}^{\prime}$, except the case $d$ even and $m_{1}=m_{2}=d / 2$. Note that if $A \in B_{2} \cup B_{2}^{\prime}$ (resp. $\left.A \in B_{3} \cup B_{3}^{\prime}\right)$, then $\left.\sharp(A \cap S)\right)>3$ (resp. $\sharp(A \cap S)>4$ ) and that if $w(A)=2 d+1$, then $\sharp(A \cap S) \geq 5$, because $m_{1}+m_{2}+m_{3}+m_{4} \leq \min \left\{4 m_{1}, 3 d-2 m_{1}\right\}$. Take a line $L \subset N$. If $w(N)=2 d+1$ and $m_{1} \leq d-1$, then $\sharp(S \cap(N \backslash L)) \geq 2$.

Remark 7. Take lines $L_{1}, L_{2}, L_{3}$ such that $L_{i} \cap L_{j}=\emptyset$ for all $i \neq j$. If $w\left(L_{1}\right)+w\left(L_{2}\right)+w\left(L_{3}\right) \geq 3 d+1$, then every element of $B_{1}$ meets $S \cap\left(L_{1} \cup\right.$ $\left.L_{2} \cup L_{3}\right)$. If $w\left(L_{1}\right)+w\left(L_{2}\right)+w\left(L_{3}\right) \geq 3 d+2$, then every element of $B_{1}^{\prime}$ meets $S \cap\left(L_{1} \cup L_{2} \cup L_{3}\right)$.

Lemma 4. Assume the existence of a hyperplane $U$ such that $w(U)=$ $3 d+1$. Then $\operatorname{Res}_{U}(Z)$ satisfies the Segre condition in degree $d-1$.

Lemma 5. Let $E, A \subset \mathbb{P}^{4}$ such that $E \cap A$ is a single point, o. Then $h^{1}\left(\mathcal{I}_{A \cup E}(t)\right)=0$ for all $t \geq 2$ and $\mathcal{I}_{A \cup E}(2)$ is spanned.

Proof. Fix lines $L, D$ of $\mathbb{P}^{3}$ such that $L \cap D=\emptyset$. We have $h^{1}\left(\mathcal{I}_{L \cup D}(2)\right)=0$, $h^{0}\left(\mathcal{I}_{A \cup D}(2)\right)=4$ and $\mathcal{I}_{L \cup D}(2)$ is spanned. Since $\left|\mathcal{I}_{A \cup D}\right|$ is the linear system of all quadric cones with vertex containing $o$, we get $h^{1}\left(\mathcal{I}_{A \cup D}(2)\right)=0$ and that $\mathcal{I}_{A \cup E}(2)$ is spanned outside $o$. Take affine coordinates $x_{1}, x_{2}, x_{3}, x_{4}$ centered at $o$ such that the affine part of $A$ (resp. $E$ ) has equations $x_{1}=x_{2}=0$ (resp. $x_{3}=x_{4}=0$ ). Since the quadratic equations $x_{i} x_{j}, i=1,2, j=3,4$, gives at $o$ a reduced scheme, we get that $\mathcal{I}_{A \cup E}(2)$ is spanned at $o$. The case $t>2$ follows from Castelnuovo-Mumford's lemma.

Lemma 6. Fix a plane $E \subset \mathbb{P}^{4}$ and lines $L, D$ such that $E \cap L=E \cap D=$ $L \cap D=\emptyset$. Then $h^{1}\left(\mathcal{I}_{E \cup L \cup D}(2)\right)=0$.

Proof. Set $H:=\langle D \cup L\rangle$. Since $H \cap E, L$ and $D$ are 3 disjoint lines of $H$, we have $h^{0}\left(H, \mathcal{I}_{(E \cap H) \cup L \cup D \cup\{p\}}(2)\right)=0$. For a general $p \in H$. To prove the lemma it is sufficient to prove that $h^{0}\left(\mathcal{I}_{E \cup L \cup D \cup\{p\}}(2)\right)=0$. $\left|\mathcal{I}_{E \cup L \cup D \cup\{p\}}(2)\right|$ is the linear system of all quadrics $H \cup M$ with $M$ a hyperplane containing $p$.

We say that $Z$ or $\left(m_{1}, \ldots, m_{s}\right)$ is not in $\diamond$ (or not with $\diamond$ ), if for every $L \in B_{1}$ we have $\sharp(L \cap S) \geq 3$, i.e. if $m_{1}+m_{2} \leq d$.

In sections $3,4,5,6$ and 7 we prove Lemma 1 . From now on we fix an integer $d \geq 4$ and assume that the generalized Segre condition is true in $\mathbb{P}^{4}$ with respect to the integers $d-1$ and $d-2$. We use induction on the integer $w\left(\mathbb{P}^{4}\right)$, i.e. we assume that the lemma is true for all $\left(S^{\prime}, Z^{\prime}\right)$ satisfying the 
Segre conditions and not in $\diamond$ with $w_{Z^{\prime}}\left(\mathbb{P}^{4}\right)<w\left(\mathbb{P}^{4}\right)$. We do not prove it in this paper, because we prove that it holds with respect to $d$ only for certain multiplicities (i.e. we exclude $\diamond$ and $\mathbf{\uparrow}$ ), but we adapt the steps so that many of them may be used even in case and some of them for $\diamond$.

Remark 8. Let $L_{i} \subset \mathbb{P}^{4}, i=1,2,3$, be lines such that $L_{i} \cap L_{j}=\emptyset$ for all $i \neq j$. There is a unique line $J$ intersecting every line $L_{1}, L_{2}, L_{3}$ and $J \cup L_{1} \cup L_{2} \cup L_{3}$ is the base locus of $\left|\mathcal{I}_{L_{1} \cup L_{2} \cup L_{3}}(2)\right|$.

\section{Extremal Hyperplanes and Lines}

In this section we assume the existence of $M \in B_{3}$ and $L \in B_{1}$ such that $M \cap L \cap S=\emptyset$. We assume $m_{1}+m_{2} \leq d$, but we point out the single steps that work with weaker assumptions.

Since $w\left(\mathbb{P}^{4}\right) \leq 4 d+1$, we have $w(M) \leq 3 d$. Until step (k) we assume $S \subset M \cup L$ (this is always the case if $w(M)=3 d$ ). Fix any $A \in B_{c} \cup B_{c}^{\prime}, A \neq L$, which is not contained in $M$. If $S \subset M \cup L$, then $A \cap S=(A \cap M \cap S) \sqcup(A \cap L \cap S)$ with $A \cap M \cap S$ contained in the hyperplane $M \cap A$ of $A$.

(a) Assume $c=2$ and $A \supset L$. We get $w(A \cap M \cap S)=w(A)-d-1$ and hence $A \cap M \cap S \neq \emptyset$. If $w(A)=2 d+1$, we get $A \cap M \in B_{1}^{\prime}$. If $w(A)=2 d$, then $w(A \cap M)=d-1$.

(b) Assume $c=3$ and $A \supset L$. The plane $A \cap M$ satisfies $w(A \cap M)=$ $w(A)-d-1$.

(c) Assume $c=1$ and $A \neq L$. Since $m_{1} \leq d-1$ (Lemma 3), we get $\sharp(A \cap M \cap S)=1, \sharp(A \cap L \cap S)=1$ and $m_{A \cap M \cap S}+m_{A \cap L \cap S}$. Hence $m_{1}+m_{2}=$ $d+1$. This case may occur only if $m_{1}+m_{2}=d+1$.

(d) Assume $c=2$ and $A \nsubseteq L$. Since $A \cap M$ is a line, we have $w(A \cap M) \leq$ $d+1$. Since $A \cap S$ spans $A$, we get $A \cap L \neq \emptyset$ and that $A \cap L$ is a point of $S$. We get $w(A)=w(A \cap M)+m_{A \cap L}$. We exclude the case $w(A)=2 d+1$, because $h^{1}\left(\mathcal{I}_{Z}(d)\right)=0$ if $m_{A \cap L}=d$ (Lemma 3). If $w(A)=2 d$, we get $m_{A \cap L} \geq d-1$. Since $d \geq 4$, we are in the case $\$$ and $A \cap L$ is the only point of $S$ with multiplicity $m_{1}=d-1$.

(e) Assume $c=3$ and $A \nsupseteq L$. Since $A \cap S$ spans $A$, we get that $A \cap L$ is a point of $L$. We get $w(A)=w(A \cap M)+m_{A \cap L}$. Write $w(A)=3 d+e$ with $e \in\{-1,0,1\}$. Since $w(A \cap M) \leq 2 d+1$, we get $m_{A \cap L} \geq d+e-1$. Lemma 3 solves the case $e=1$. If $e \leq 0$ we get $m_{A \cap L} \geq d-1+e$. If either $d \geq 5$ or $d=4$ and $e=0$, we are in case $\boldsymbol{\uparrow}$ and $A \cap L$ is the only point of $S$ with multiplicity $m_{1}$. If $d=4$ and $e=-1$ we get $m_{A \cap L} \geq 2$. 
(f) Assume the existence of a plane $E \subset M$ with $w(E)=2 d+1$. We only exclude the case $d$ odd, $m_{1}=m_{2}=m_{3}=(d+1) / 2$ with $E \cap S$ formed by 3 points with multiplicity $(d+1) / 2$ and one point with multiplicity $(d-1) / 2$ and the case $d$ even, $m_{1}=d / 2+1$ and $E$ containing the point of multiplicity $d / 2+1$ and 3 points of multiplicity $d / 2$. With these exclusions we have $\sharp(E \cap S) \geq 5$. We prove that $h^{1}\left(\mathcal{I}_{Z}(d)\right)=0$. Fix $q \in L \cap S$ with maximal multiplicity among the points of $L \cap S$ and set $H:=\langle E \cup\{q\}\rangle$. We check that $\operatorname{Res}_{H}(Z)$ satisfies the Segre conditions in degree $d-1$, and hence that $h^{1}\left(\mathcal{I}_{Z}(d)\right)=0$ by induction on $d$. We have $w_{\operatorname{Res}_{H}(Z)}\left(\mathbb{P}^{4}\right) \leq 4 d+1-\sharp(S \cap L \cap H)-\sharp(E \cap S)$ and hence to check the Segre condition in degree $d-1$ for $\operatorname{Res}_{H}(Z)$ and $\mathbb{P}^{4}$ it is sufficient to use that $\sharp(E \cap S) \geq 3$. $M$ satisfies the Segre condition in degree $d-1$ for $\operatorname{Res}_{H}(Z)$, because $w(M) \leq 3 d$ and $\sharp(M \cap H \cap S)=\sharp(E \cap S) \geq 3$. Since $L \cap H \cap S \neq \emptyset, L$ satisfies the Segre condition in degree $d-1$ for $\operatorname{Res}_{H}(Z)$. If $R \in B_{1}, R \neq L$, and $R \nsubseteq M$, then (c) shows that $R$ satisfies the Segre condition in degree $d-1$ for $\operatorname{Res}_{H}(Z)$. Fix $A \in B_{2}$ with $A \supset L$. Since $S \cap H \cap L \neq \emptyset$, to check the Segre condition for $A$ we may assume $w(A)=2 d+1$; in this case it is sufficient to check that $A \cap E \cap S \neq \emptyset$; we have $w(A \backslash L)=d$; since $w(M \backslash E) \leq d-1$, we get $A \cap E \cap S \neq \emptyset$. Fix $A \in B_{3}$ such that $A \supset L$. Since $L \cap H \cap A \cap S \neq \emptyset$, it is sufficient to prove that $\sharp(E \cap A \cap S) \geq w(A)-3 d+1$. We have $w(A \cap M \cap S)=w(A)-d-1$ and hence it is sufficient to use that $w(M \backslash E) \leq d-1$ and that if $w(A)=3 d+1$, then $w(A \cap E \cap S) \geq d+1$ and hence $\sharp(A \cap E \cap S) \geq 2$.

Fix $D \in B_{1}$ such that $D \subset M$. Since $w(M \backslash D)>w(E)$, we have $D \cap E \cap S \neq$ $\emptyset$. Fix $A \in B_{2}$ with $A \subset M$. Since $w(A) \geq d+w(M \backslash E)$, we have $\sharp(A \cap S) \geq 2$, concluding the proof that $\operatorname{Res}_{H}(Z)$ satisfies the Segre conditions in degree $d-1$.

(g) Assume the existence of $R, D \in B_{1}$ such that $R \subset M, D \subset M$ and $R \cap D=\emptyset$. We assume that either $m_{1}+m_{2} \leq d$ or $m_{1}>m_{2}$ and the only point with multiplicity $m_{1}=d+1-m_{2}$ is contained in $L$. Hence $\sharp(B \cap S) \geq 3$ for each $B \in B_{1}$ with $B \subset M$. Fix $p, q \in L \cap S$ with $p \neq q$ and with maximal multiplicity among the points of $L \cap S$. Let $J \subset M$ be the only line containing the point $M \cap L$ and intersecting both $R$ and $D$ (it is the only line of $\mathbb{P}^{4}$ intersecting the 3 lines $L, D, R) . \quad L \cup D \cup R \cup J$ is the base locus $\left|\mathcal{I}_{L \cup D \cup R}(2)\right|$. Since $M \cap L \notin S, D \cup R \cup\{p, q\}$ is the base locus of $\left|\mathcal{I}_{D \cup R \cup\{p, q\}}(2)\right|$. Therefore there is $Q \in\left|\mathcal{I}_{D \cup R \cup\{p, q\}}(2)\right|$ such that $Q \cap S \nsubseteq S$. By the inductive assumption we get $h^{1}\left(\mathcal{I}_{\sum_{o \in S \cap Q} m_{o}}(d)\right)=0$ and hence $h^{1}\left(Q, \mathcal{I}_{Z \cap Q}(d)\right)=0$. Therefore it is sufficient to check the Segre condition for $\operatorname{Res}_{Q}(Z)$ in degree $d-2$. Since $p, q \in S$, to check the condition for $\mathbb{P}^{4}$ it is sufficient to have $\sharp(D \cap S)+\sharp(R \cap S) \geq 6$. This is true by our assumptions on $m_{1}, m_{2}$. By construction $\operatorname{Res}_{Q}(Z)$ satisfies the Segre condition in degree $d-2$ for $L, R, D$ and, since $\sharp(D \cap S)+\sharp(R \cap S) \geq 6$, 
for $M$. Every $B \in B_{1} \backslash\{L\}$ is contained in $M$ and meets both $D$ and $R$, so that $w_{\operatorname{Res}_{Q}(Z)}(B) \leq d-1$; by Remark 5 every $B \in B_{1}^{\prime}$ with $B \subset M$ meets $D \cup R$ and hence it meets $(D \cup R) \cap S$ (Remark 3$)$, so that $w_{\operatorname{Res}_{Q}(Z)}(B) \leq d-1$. Take $B \in B_{1}^{\prime}$ with $B \nsupseteq M$; since $B \cap S=(M \cap B \cap S) \sqcup(L \cap M \cap S)$, there are $o \in M \cap S$ and $u \in L \cap S$ with $B=\langle\{o, u\}\rangle$ and $m_{o}+m_{u}=d$; we have $u \in\{p, q\}$, because in case $m_{1}+m_{2}=d+1$ we assumed $m_{1}>m_{2}$ and that the only point with multiplicity $m_{1}$ is contained in $L$, while if $d$ is even and $m_{o}=m_{u}=d / 2$, then $u$ is one of the points (at most two) with maximal multiplicity in $L \cap S$.

(g1) Fix $A \in B_{2} \cup B_{2}^{\prime}$ such that $A \supset L$. Since $p, q \in A \cap Q \cap S$, to check the Segre condition for $A$ we may assume $A \in B_{2}$. We have $w(A \backslash L)=$ $w(A)-d-1>w(M \backslash(D \cup R))$. Hence $A \cap S \cap(D \cup R) \neq \emptyset$, say $A \cap D \cap S \neq \emptyset$. Therefore to check the Segre condition for $A$ we may assume $w(A)=2 d+1$; we only need to check the case $\sharp(L \cap S)=2$, i.e. $\boldsymbol{\phi}, m_{1}+m_{2}=d+1$, the point with maximal multiplicity is in $L$ and $\sharp(A \cap(R \cup D) \cap S)=1$, say $R \cap A \cap S=\emptyset$; since $w(\langle A \cup R\rangle) \geq 3 d+2$, the hyperplane $\langle R \cup A\rangle$ contradicts the Segre conditions for $Z$.

(g2) Fix $A \in B_{2} \cup B_{2}^{\prime}$ such that $A \nsubseteq L$ and $A \nsubseteq M$. $A$ is spanned by $A \cap M \cap S$ and $A \cap L \cap S$ and hence $A \cap L \in S$ and $m_{A \cap L}+w(A \cap M)=w(A)$. We use Proposition 2 for the case $d=4$ and $m_{A \cap L}=1$. Now assume that either $d \geq 5$ or $m_{A \cap L}>1$. Since $w(A)-w(A \cap M) \geq 2 d-2-d-1=d-3$, if $d \geq 5$ we get that $m_{A \cap L} \in\{p, q\}$, while this is true if $d=4$, because $p, q$ have maximal multiplicity. By Lemma 3 we may assume $m_{A \cap L} \leq d-1$. Since $w(A \cap M) \leq d+1$, we get $w(A) \leq 2 d$ and that if $w(A)=2 d$. First assume $w(A)=2 d$. We get $m_{A \cap L}=d-1$ and $B:=A \cap M \in B_{1}$. Hence $m_{2} \leq 2$. Since $Q \supset D \cup R$, it is sufficient to prove that $D \cap B \cap S \neq \emptyset$ and $R \cap B \cap S \neq \emptyset$. Assume for instance $D \cap B \cap S=\emptyset$. Since the point of $D \cap B \cap S$ has multiplicity $\leq 2$, we get $3 d \geq w(M) \geq 3(d+1)-2$, a contradiction. If $w(A)=2 d-2$, it is sufficient to use that $A \cap L \in\{p, q\}$. Now assume $w(A)=2 d-1$. Since $A \cap L \in\{p, q\}$, it is sufficient to prove that $A \cap(D \cup R) \cap S \neq \emptyset$. Since $m_{1} \leq d-1$, we have $w(A \cap M) \geq d$ and (since $M \cap L)=\emptyset, A \cap(D \cup R) \cap S \neq \emptyset$ by Remark 7.

(g3) Fix $A \in B_{2} \cup B_{2}^{\prime}$ contained in $M$. We need to check that $\sharp(A \cap S \cap(R \cup$ $D)) \geq w(A)+3-2 d$. Since $w(M \backslash(D \cup R)) \leq d-2$, we have $A \cap S \cap(R \cup D) \geq$ $w(A)-d+2$. Since $w(A) \geq 2 d-2$, then we get $A \cap S \cap(R \cup S D) \neq \emptyset$. If $w(A)=2 d-1$, we get $\sharp(A \cap S \cap(R \cup D)) \geq 2$. If $w(A)=2 d$, we get $\sharp(A \cap S \cap(R \cup D)) \geq 3$. Now assume $w(A)=2 d+1$. This case is solved in step (f).

(g4) Fix $A \in B_{3} \cup B_{3}^{\prime}$. If $A=M$, we use that $\sharp(D \cap S) \geq 3$ and $\sharp(R \cap S) \geq 3$. Assume $A \supset L$; since $\{p, q\} \in L$, we may assume $w(A) \geq$ 
$3 d-1$; since $w(A \backslash L)=w(A)-d-1$ and $w(M \backslash(D \cup R)) \leq d-2$, we get $w(A \cap(D \cup R)) \geq w(A)-2 d+1>0$; hence we may assume $w(A) \geq 3 d$; we get $\sharp(S \cap A \cap(D \cup R)) \geq 2$, and hence we may assume $w(A)=3 d+1$; we get $\sharp(S \cap(D \cup R)) \geq 3$, because either $m_{1} \leq d / 2$ or with $L$ containing the point with multiplicity $m_{1}$; hence $A$ contains at least two points of one of the lines $D$, $R$ (say $D$ ); since $\sharp(S \cap D) \geq 3$, it is sufficient to check that $S \cap R \cap A \neq \emptyset$; this is true, because $m_{p}+m_{q}+w(D)+w(M \backslash(R \cup D)) \leq d+d+1+d-2<w(A)$.

Now assume $A \neq M$ and $A \nsupseteq L$. We have $w(A)=w(A \cap M)+m_{A \cap L}$. By step (f) we may assume $w(A \cap M) \leq 2 d$. Since $A \cap S$ spans $S$ (Remark 2 ), we have $m_{A \cap L}>0$. If $w(A) \geq 3 d$, then $w(A \cap M) \geq 2 d+1$, because $m_{1} \leq d-1$ (Lemma 3) and hence we conclude by step (f). If $w(A)=3 d-1$, then we get $m_{1}=d-1, m_{2} \leq 2, w(A \cap M)=2 d$ and $A \cap L \in\{p, q\}$; since $w(M \backslash(D \cup R)) \leq d-2$, we get $w(A \cap(D \cup R)) \geq d+2$ and hence $\sharp(A \cap(R \cup D)) \geq 3$, so that $w_{\operatorname{Res}_{Q}(Z)}(A) \leq 3 d-5$. Hence we may assume $3 d-4 \leq w(A) \leq 3 d-2$. First assume $A \cap L \notin\{p, q\}$; we get $m_{A \cap L} \leq\lfloor(d+1) / 3\rfloor$; since $w(A \cap M) \leq 2 d$, we get $d<4$ (a contradiction) if $w(A)=3 d-2$, $4 \leq d \leq 5$ if $w(A)=3 d-3$ and $4 \leq d \leq 6$ if $w(A)=3 d-4$; in these exceptional cases we have $w(A \cap M)=2 d$ and so $w(A \cap(D \cup R)) \geq d+2$ and $\sharp(A \cap(D \cup R) \cap S) \geq 3$ (since only $L$ may have a point with multiplicity $>d / 2$, and hence $w_{\operatorname{Res}_{Q}(Z)}(A) \leq 3 d-5$. Since $\{p, q\} \in A$, we may assume $3 d-3 \leq w(A) \leq 3 d-2$; in the first case we need to prove that $A \cap(D \cup R) \cap S \neq \emptyset$, while in the second case we need $\sharp(A \cap(D \cup R) \cap S) \geq 2$. If $w(A)=3 d-3$, it is sufficient to have $m_{1}+d-2 \leq 3 d-4$, which is true for all $d \geq 4$. Assume $w(A)=3 d-2$. It is sufficient to use that $2 d-1 \leq 3 d-2$ and hence $m_{1}+m_{2}<w(A)-w(M \backslash(D \cup R))$.

(g5) Assume the existence of $T \in B_{1} \cup B_{1}^{\prime}$ with $T \neq L$ and $T \nsubseteq M$; we get either with or $d$ even, $m_{1}=m_{2}=d / 2$ and $m_{p}=d / 2$; in the latter case $p \in B$ and hence $w_{\operatorname{Res}_{Q}(Z)}(T) \leq d-1$.

(g6) Fix $T \in B_{1} \cup B_{1}^{\prime}$ such that $T \subset M$; since $R$ and $D$ satisfy the Segre condition for $\operatorname{Res}_{Q}(Z)$ in degree $d-2$, we may assume $T \neq D$ and $T \neq R$; since $w(M \backslash(R \cup D)) \leq d-2$, we get $T \cap S \cap(R \cup D) \neq \emptyset$; hence if either $T \in B_{1}^{\prime}$ or $T \cap S$ meets both $D$ and $R$, then $T$ satisfies the Segre condition in degree $d-2$, but not if either $T \cap D \cap S=\emptyset$ or $T \cap R \cap S=\emptyset$. With no loss of generality we may assume $T \cap R \cap S=\emptyset$. Since $T, R \in B_{1}$, we have $T \cap R=\emptyset$ (Remark 3).

Claim 1: Fix any $p_{1}, p_{2} \in L \backslash(L \cap M)$. Then $h^{1}\left(\mathcal{I}_{T \cup D \cup R \cup\left\{p_{1}, p_{2}\right\}}(2)\right)=0$, i.e. $h^{0}\left(\mathcal{I}_{T \cup D \cup R \cup\left\{p_{1}, p_{2}\right\}}(2)\right)=5$.

Proof of Claim 1: Any two configurations $T, D, R$ inside a projective space $M$ are projectively equivalent and $h^{1}\left(M, \mathcal{I}_{T \cup D \cup R, M}(2)\right)=0$. Hence 
$h^{1}\left(\mathcal{I}_{T \cup D \cup M}(2)\right)=0$. We have $h^{1}\left(\mathcal{I}_{\left\{p_{1}, p_{2}\right\}}(1)\right)=0$. Since $p_{i} \neq L \cap M$, using reducible quadrics with $M$ as a component, we get $h^{1}\left(\mathcal{I}_{T \cup D \cup R \cup\left\{p_{1}, p_{2}\right\}}(2)\right)=0$.

Fix $o \in T \cap S \backslash D \cap S$. We have $h^{0}\left(\mathcal{I}_{T \cup D \cup R \cup\{p, q\}}(2)\right)=h^{0}\left(\mathcal{I}_{D \cup R \cup\{o, p, q\}}(2)\right)-1$ (Claim 1) and hence there is $Q^{\prime} \in\left|\mathcal{I}_{D \cup R \cup\{o, p, q\}}(2)\right|$ with $T \nsubseteq Q^{\prime}$. Since $T \cap Q^{\prime}$ contains two points of $T$, $o$ and $D \cap T$, we get $T \cap Q^{\prime}=\{o, D \cap T\}$. Since $\sharp(T \cap S) \geq 3$, we have $Q^{\prime} \cap S \neq S$. Therefore $h^{1}\left(\mathcal{I}_{Q^{\prime} \cap S}(2)\right)=0$. We may repeat the construction just given and see that $\operatorname{Res}_{Q^{\prime}}(Z)$ satisfies the Segre conditions in degree $d-2$, except perhaps for the lines like $T$, i.e. the lines $A \in B_{1}$ with $A \subset M$ and $A$ intersecting only one of the lines $R, D$. The choice of $o$ gives that $T$ satisfies the Segre condition in degree $d-2$. Take another line $A \neq T$. Since every point of $M$ has multiplicity $\leq d / 2$, we have $w(T \backslash T \cap(D \cup R)) \geq d / 2+1$ and the same inequality is true for $A$. Since $w(M \backslash(D \cup R)) \leq d-2<w(T \backslash T \cap(D \cup R))+w(A \backslash A \cap(D \cup R)), T$ and $A$ meets in a point of $S$ not contained in $D \cap T$. We may take as $o$ this point and handle simultaneously $A$ and $T$. So the only problem arises if there is a third line $T^{\prime}$ and $o \notin T^{\prime}$. We just saw that $T \cap T^{\prime} \neq \emptyset$ and $T^{\prime} \cap A \neq \emptyset$, so that $T^{\prime}$ (and any element of $B_{1}$ with the same properties, but not containing $o$ ) is contained in the plane $\langle A \cup T\rangle$. We first note that $T \cap T^{\prime} \cap(D \cup R)=\emptyset$, because $T$ and $T^{\prime}$ are different lines containing a point outside $D \cup R$. Hence the plane $\langle A \cup T\rangle$ contains at least two points of one of the lines $D, R$, say of $D$, and hence it contains $D$. Therefore $\langle A \cup T\rangle=\langle D \cup T\rangle$ and $R$ is disjoint from $T, A, T^{\prime}$ and all other elements of $B_{1}$ with the same properties. Take a general $Q_{1} \in\left|\mathcal{I}_{T \cup D \cup R \cup\left\{p_{1}, p_{2}\right\}}(2)\right|$. We have $\sharp\left(A \cap S \cap Q_{1}\right) \geq 2$, because $A \cap D \neq A \cap T$ and any two mutually intersecting elements of $B_{1}$ meets in a point of $S$ (Remark 3 ). Hence $A$ (and any other element of $B_{1}$ with similar properties) satisfies the Segre conditions in degree $d-2$ with respect to $\operatorname{Res}_{Q_{1}}(Z)$. The problem is that usually $Q_{1} \cap S=S$, because (since $R$ meets the hyperplane $\langle A \cup T\rangle$ of $M$ ) we have $\langle A \cup T\rangle \subset Q_{1}$. This is the base locus of $\left|\mathcal{I}_{\langle T \cup D\rangle \cup L \cup R}(2)\right|$ and so we conclude unless $S \subset\langle T \cup D\rangle \cup L \cup R$. From now on we assume $S \subset\langle T \cup D\rangle \cup L \cup R$.

Let $Q_{2}$ be the reducible quadric hypersurface union of the hyperplane $\langle T \cup$ $D \cup\{p\}\rangle$ and the hyperplane $\langle\{q, o\} \cup R\rangle$. Since $\{p, q\} \cup R \cup\langle T \cup D\rangle \subset Q_{2}$ the proofs just given for $\operatorname{Res}_{Q}(Z)$ works (even for all $B \in B_{1}$ with $B \subset\langle T \cup D\rangle$ ). We have $Q_{2} \cap S \neq S$, because $Q_{2} \cap L=\{L \cap M, p, q\}$, unless $\boldsymbol{A}$ and the only point of $S$ with multiplicity $m_{1}$ is contained in $L$; in this case we call $p$ this point and instead of $Q_{2}$ we use the reducible quadric $Q_{3}$ union of the hyperplane $\langle T \cup D \cup\{p\}\rangle$ and the hyperplane $\langle\{p, o\} \cup R\rangle$; the point $p$ has multiplicity $m_{p}-2$ in $\operatorname{Res}_{Q_{3}}(Z)$ and hence the proof just given works even in the case $A \supseteq L$.

(h) Assume the existence of $R \in B_{1}$ such that all $B \in B_{1}$ and contained in $M$ meet $R$. Set $H:=\langle L \cup R\rangle$. It is sufficient to prove that $\operatorname{Res}_{H}(Z)$ satisfies 
the Segre conditions in degree $d-1$. We have $w_{\operatorname{Res}_{H}(Z)}\left(\mathbb{P}^{4}\right) \leq 4 d+1-\sharp(S \cap$ $R)-\sharp(S \cap L)$. We have $w_{\operatorname{Res}_{H}(Z)}(B) \leq d+1-\sharp(B \cap S)$ if $B \in\{R, L\}$. Now take $B \in B_{1} \backslash\{R, L\}$. If $B \subset M$, then $B \cap R \neq \emptyset$ by assumption and $B \cap R \cap S \neq \emptyset$ (Remark 3) and so $w_{\operatorname{Res}_{H}(Z)}(B) \leq d$. If $B \nsubseteq M$, then $B \cap L \cap S \neq \emptyset$, because $S \subset M \cup L$ and so $w_{\operatorname{Res}_{H}(Z)}(B) \leq d$.

(h1) Fix $A \in B_{2}$. If $A \subset M$, then we may assume $w(A)=2 d$ by step (f). We have $A \cap R \cap S \neq \emptyset$, because $w(M) \leq 3 d$ and hence $w(M \backslash R) \leq 2 d-1$. If $A \nsubseteq M$, then we have $w(A \cap M) \leq d+1$ and if equality holds, then $A \cap R \cap S \neq \emptyset$ by our assumption on $B_{1}$. If $A \supset L$, then we use that $\sharp(L \cap S) \geq 2$. If $A \nsupseteq L$, we get $A \cap L \in S$ and $m_{A \cap L}+w(A \cap M)=w(A)$; since $m_{A \cap L} \leq d-1$, we get $w(A \cap M)=d+1, w(A)=2 d$ and $A \cap S \cap H \neq \emptyset$; hence $A$ satisfies the Segre condition for $\operatorname{Res}_{H}(Z)$.

(h2) Take $A \in B_{3}$. If $A=M$, then we use that $R \cap S \subset H$. Now assume $A \supset L$; since $\sharp(L \cap S) \geq 2$, we are done, unless $w(A)=3 d+1$, and $L$ contains a point with maximal multiplicity; since $S \subset M \cup L$, we get $w(A \cap M)=2 d$; since $w(M \backslash R)=w(M)-d-1 \leq 2 d-1$, we get $A \cap R \cap S \neq \emptyset$ and hence $\sharp(A \cap H \cap S) \geq 3$ even in this case. Now assume $A \neq M$ and $A \nsubseteq L$; since $S \subset M \cup L$, we have $w(A)=w(A \cap M)+m_{A \cap L}$; by step (f) we may assume $w(A \cap M) \leq 2 d$; since $m_{A \cap L} \leq d-1$ (Lemma 3 ), we conclude, unless $w(A)=3 d-1$ and $m_{A \cap L}=d-1$; in this case the Segre condition is satisfied, because $A \cap L \in S \cap H$.

(i) By Steps (f), (g) and (h) we may assume that $M$ contains no element of $B_{1}$ and no $A \in B_{2}$ with $w(A)=2 d+1$. Assume the existence of $E \in B_{2}$ with $E \subset M$ and $w(E)=2 d$. Fix $p \in L \cap S$ with maximal multiplicity and set $H:=\langle E \cup\{p\}\rangle$. We have $w_{\operatorname{Res}_{H}(Z)}\left(\mathbb{P}^{4}\right) \leq 4 d+1-\sharp(S \cap L \cap H)-\sharp(E \cap S)$ and hence to check the Segre conditions in degree $d-1$ for $\operatorname{Res}_{H}(Z)$ is is sufficient to use that $\sharp(E \cap S) \geq 3$ (Remark 2). Since $q \in L \cap S, L$ satisfies the Segre condition in degree $d-1$. By assumption we do not need to test any other element of $B_{1}$.

(i1) Fix $A \in B_{3}$. If $A=M$, then we use that $\sharp(E \cap S) \geq 3$. Now assume $A \neq M$ and $A \nsupseteq L$; we have $w(A)=w(A \cap M)+m_{A \cap L}$; we may assume $m_{A \cap L}<d$ by Lemma $3 ; h^{1}\left(\mathcal{I}_{Z}(d)\right)=0$ if $w(A \cap M)=2 d+1$ by step (f); hence $h^{1}\left(\mathcal{I}_{Z}(d)\right)=0$ in this case (if $m_{1}=d-1$, then $m_{p}=d-1 \in A \cap H \cap S$ ). Now assume $A \supset L$; since $p \in H \cap A \cap S$, to check the Segre condition we may assume $w(A) \geq 3 d$; we get $w(A \cap M) \geq 2 d-1$ and hence $E \cap A \cap S \neq \emptyset$; to check the Segre condition we may assume $w(A)=3 d+1$ and in this case we use Lemma 4.

(i2) Fix $A \in B_{2}$. First assume $A \subset M$. By step (f) we may assume $w(A)=2 d$ and it is sufficient to prove that $A \cap H \cap S \neq \emptyset$; we have $w(A \backslash A \cap E) \leq$ 
$w(M)-w(E) \leq d$ and hence $A \cap E \cap S \neq \emptyset$. Now assume $A \nsubseteq M$ and $A \nsupseteq L$; since $S \subset M \cup S$, we get $A \cap L \cap S \neq \emptyset$ and $w(A)=w(A \cap M)+$ $m_{A \cap L}$, contradicting the assumption $w(A \cap M) \leq d$, because if $m_{A \cap L}=d$, then $h^{1}\left(\mathcal{I}_{Z}(d)\right)=0$ by Lemma 3. Now assume $A \supset L$. Since $p \in H$, to check the Segre condition in degree $d-1$ for $A$ we may assume $w(A)=2 d+1$ and hence $w(A \cap M)=d$. Therefore $B:=A \cap M \in B_{1}^{\prime}$. In this case $S=$ $(S \cap E) \sqcup(S \cap A \cap M) \sqcup S \cap L$. Let $F$ be the hyperplane spanned by $A$, and a point $o \in E \cap S$ with maximal multiplicity. We need to check the Segre conditions of $\operatorname{Res}_{F}(Z)$ in degree $d-1$. Since $\sharp(A \cap S) \geq \sharp(B \cap S)+\sharp(A \cap L \cap S)$, the Segre condition for $\mathbb{P}^{4}$ is satisfied. Fix $U \in B_{3}$. If $U=M$, then we use that $w(M) \leq 3 d$ and $\sharp(A \cap M \cap S) \geq 2$. If $U \supset L$, then we use that $\sharp(L \cap S) \geq 3$, unless $\boldsymbol{\uparrow}$ and $L$ and $L$ contains the point with maximal multiplicity; in the latter case we may assume $w(U)=3 d+1$ (and conclude by Lemma 4), unless $U \cap M=E$; in this case we use that $w(U \cap M)=2 d, w(M \backslash A) \leq d$ and hence $w(U \cap M \cap A)>0$.

If $U \nsubseteq L$, then $w(U)=w(U \cap E)+w(U \cap B)+m_{U \cap L}$ and hence $U \cap L \in S$; we may assume $w(U) \geq 3 d$ and, since $m_{U \cap L}<d$ by Lemma 3 , we may assume $w(U)=3 d+1$; use Lemma 4 .

Fix $U \in B_{2}$. If $U=E$, then we use $F \cap E \cap S \neq \emptyset$ and that $w(E)=2 d$. If $U \subset M$ and $U \neq M$, then we use that $w(U)=2 d$ by step (f) and that $U \cap B \cap S \neq \emptyset$, because $w(U)+w(E)>w(M)$. If $U \supset L$, then we use that $\sharp(L \cap S) \geq 2$. If $U \nsubseteq M$ and $U \nsupseteq L$, then $w(U)=w(U \cap M)+w(U \cap L)$ with $w(U \cap M) \leq d$; we apply Lemma 3 to the point $U \cap L$.

Fix $U \in B_{1}$. Since $M$ contains no element of $B_{1}$, it is sufficient to observe that $S=(S \cap M) \cup(S \cap L)$ and hence $U \cap L \cap S \neq \emptyset$.

(j) Assume that $M$ contains no element of $B_{1} \cup B_{2}$. We take $p, q \in M \cap S$ and take a hyperplane $H$ containing $L \cup\{p, q\}$. We heck the Segre conditions in degree $d-1$ with respect to $\operatorname{Res}_{H}(Z)$. To test $\mathbb{P}^{4}$ it is sufficient to use that $p, q \in H \cap S$ and that $\sharp(L \cap S) \geq 2$. Fix $U \in B_{3}$. If $U=M$, then we use that $w(M) \leq 3 d$ and that $p, q \in S$. If $U \neq M$, then we use that $w(U)=w(U \cap M)+w(M \cap L)$ with $w(U \cap M) \leq 2 d-1$; since $m_{1}<d$, we get $M \supset L$; in the case $\sharp(L \cap S)=2$, to check the Segre conditions we may assume $w(U)=3 d+1$, which gives $w(U \cap M)=2 d$, a contradiction. Fix $U \in B_{2}$. By assumption $U \nsubseteq M$. If $U \supset L$, then we use that $\sharp(L \cap S) \geq 2$. If $U \nsubseteq L$, then we get $w(U)=w(U \cap M)+m_{U \cap L}$ with $w(U \cap M) \leq d$ and so we apply Lemma 3 .

Fix $U \in B_{1}$. Since $U \nsubseteq M, U$ is spanned by $U \cap S$ and $S=(S \cap M) \cup(S \cap L)$, then $U \cap L \cap S \neq \emptyset$ and hence $w_{\operatorname{Res}_{H}(Z)}(U) \leq d$.

(k) Assume $S \nsubseteq M \cup L$, i.e. assume $w\left(\mathbb{P}^{4}\right)=4 d+1, w(M)=3 d-1$. 
We check that $h^{1}\left(\mathcal{I}_{Z}(d)\right)=0$, except at most in one case with $\diamond$. Set $S^{\prime}:=$ $S \cap(M \cup L)$. Let $o$ be the only point of $S \backslash S^{\prime}$. We have $m_{o}=1$, because $w(M)+w(L)=4 d$. Let $Q$ be a general element of $\left|\mathcal{I}_{M \cup L}(2)\right|$. Since $M \cup L$ is the base locus of $\left|\mathcal{I}_{M \cup L}(2)\right|$ and $Q$ is general, then $o \notin Q$. By the inductive assumption we have $h^{1}\left(\mathcal{I}_{Z \cap Q}(d)\right)=0$ and hence $h^{1}\left(Q, \mathcal{I}_{Z \cap Q}(d)\right)=0$. Therefore it is sufficient to prove that $\operatorname{Res}_{Q}(Z)$ satisfies the Segre conditions in degree $d-2$. Write $m_{1} \geq \cdots \geq m_{s}=1$.

Claim 2: We have $s \geq 9$.

Proof of Claim 2: We have $m_{1}+\cdots+m_{s-1}=4 d$. Hence $(s-1) m_{1} \geq 4 d$. Therefore to prove Claim 2 we may assume $m_{1} \geq\lceil(d+1) / 2\rceil$. Since $m_{1}+m_{i} \leq$ $d+1$ for all $i>1$, we get $4 d=m_{1}+\cdots+m_{s-1} \leq(s-2)(d+1)-(s-3)\lceil(d+1) / 2\rceil$. Hence $s>8$ if $d \geq 4$.

By Claim $2 w_{\operatorname{Res}_{Q}(Z)}\left(\mathbb{P}^{4}\right)=w\left(\mathbb{P}^{4}\right)-\sharp\left(S^{\prime}\right) \geq 4 d+1-9=4(d-2)+1$ and hence $\mathbb{P}^{4}$ satisfies the Segre condition in degree $d-2$ with respect to $\operatorname{Res}_{Q}(Z)$. Fix $A \in B_{c} \cup B_{c}^{\prime}, c=1,2,3$. Since $A \cap S$ spans $A$, we have $\sharp\left(A \cap S^{\prime}\right) \geq \sharp(A \cap S)-1 \geq c$. Hence if $A \in B_{c}^{\prime}$, then it satisfies the Segre condition in degree $d-2$ with respect to $\operatorname{Res}_{Q}(Z)$. Now assume $A \in B_{c}$. We have $w_{Z \backslash\{o\}}(A) \geq w(A)-1$. If $c=1$ we use that $m_{1} \leq d-1$ and hence $\sharp\left(S^{\prime} \cap A\right) \geq 2$. Now assume $c=2$ and $w(A)=2 d$. We have $\sharp\left(A \cap S^{\prime}\right) \geq 3$, because $m_{1}+m_{2} \leq d+1<2 d-1$ (if $d>3$ ). Now assume $c=2$ and $w(A)=2 d+1$. We need to check that $\sharp\left(A \cap S^{\prime}\right) \geq 4$. Assume $A \cap S^{\prime} \subseteq\left\{o_{1}, o_{2}, o_{3}\right\}$ with $m_{o_{1}} \geq m_{o_{2}} \geq m_{o_{3}}$. Since $m_{o_{1}}+m_{o_{2}} \leq d+1$, we get $2 d \leq m_{o_{1}}+m_{o_{2}}+m_{o_{3}} \leq 2(d+1)-m_{o_{1}}$. We get a contradiction if $m_{o_{1}} \geq 3$. If $m_{o_{1}} \leq 2$, we get $2 d \leq 6$, contradicting the assumption $d>3$. Now assume $c=3$ and write $A \cap S^{\prime}=\left\{o_{1}, \ldots, o_{h}\right\}$ with $m_{o_{1}} \geq \cdots \geq m_{o_{h}}$. We get

$$
w(A)-1 \leq(h-1)(d+1)-(h-2) m_{o_{1}}
$$

First assume $w(A)=3 d-1$. The inequality (2) gives $h>3$. Now assume $w(A)=3 d$. From (2) we get $h>4$ if $m_{o_{1}} \geq 3$. If $m_{o_{1}} \leq 2$ and $h \leq 4$ we get $3 d-1 \leq 8$, contradicting the assumption $d>3$. Now assume $w(A)=3 d+1$. Since $m_{1}+\cdots+m_{h} \leq h m_{o_{1}}$, we have $h \geq 6$ (and hence $A$ satisfies the Segre condition in degree $d-2$ with respect to $\left.\operatorname{Res}_{Q}(Z)\right)$ if $m_{o_{1}} \leq d / 2$. Assume $m_{o_{1}} \geq\lceil(d+1) / 2\rceil$ and $h \leq 5$. If $d=4$, we use Proposition 2. By (2) we have $3 d \leq(5 d+5) / 2$, which is false if $d \geq 7$. If $d=6$ we use that $m_{1} \geq 4$ and (2) to get $h>5$. Now assume $d=5$ and $h \leq 5$. From (2) it is sufficient to check the case $m_{o_{1}}=3$. We get $h=5$ and $m_{o_{1}}=3$ for all $i$. We are in case $\diamond$ with $m_{5}=(d+1) / 2$. We exclude this case taking a non-general $Q$. We may take as $Q$ any reducible quadric $Q^{\prime}=H \cup$ Since $L \in B_{1}$, two of the points of $S^{\prime}$ are the points of $L \cap S$. Hence all other points of $S^{\prime}$ are contained in $M$. 


\section{4. $B_{3} \neq \emptyset$}

In this section we assume $B_{3} \neq \emptyset$. We assume $m_{1}+m_{2} \leq d$. By section 3 we may assume $M \cap L \cap S \neq \emptyset$ for all $M \in B_{3}$ and all $L \in B_{1}$.

Call $H$ an element of $B_{3}$ with maximal weight. It is sufficient to prove that $W:=\operatorname{Res}_{H}(Z)$ satisfies the Segre condition in degree $d-1$. Our assumption gives that $W$ satisfies the Segre condition with respect to all lines. We have $w_{W}\left(\mathbb{P}^{4}\right)=w\left(\mathbb{P}^{4}\right)-\sharp(H \cap S) \leq 4 d+1-4$ by Remark 2 . We have $w_{W}(H) \leq$ $w(H)-4 \leq 3(d-1)$. Take $M \in B_{3}$ with $M \neq H$ (if any). Since $w\left(\mathbb{P}^{4}\right) \leq 4 d+1$, we have $w(M \cap H) \geq w(M)+w(H)-4 d-1$. Since $w(M)+w(H)-4 d-1 \geq$ $2 d-3>0$, we get $M \cap H \cap S \neq \emptyset$. Hence to check that $M$ satisfies the Segre condition for $W$ in degree $d-1$ we may assume $w(M) \geq 3 d$. Our choice of $H$ gives $w(H) \geq 3 d$. Hence $w(M)+w(H)-4 d-1 \geq 2 d-1$. Since $m_{1} \leq d-1$, we get $\sharp(M \cap H) \geq 3$.

(a) Fix $N \in B_{2}$. $N$ satisfies the Segre condition for $W$ in degree $d-1$ if and only if either $w(N)=2 d$ and $N \cap H \cap S \neq \emptyset$ or $w(N)=2 d+1$ and $\sharp(N \cap H \cap S) \geq$ 2. Since $w\left(\mathbb{P}^{4}\right) \leq 4 d+1$, we have $w(N \cap H) \geq w(M)+w(N)-4 d-1$. Hence $w(N \cap H)>0$, i.e. $N \cap H \cap S \neq \emptyset$. Thus to check the Segre condition for $W$ we may assume $w(N)=2 d+1$. We get $w(N \cap H) \geq w(H)-2 d$. We get $\sharp(N \cap H) \geq 2$, unless either $w(H)=3 d-1, m_{1}=d-1$ and $N \cap H$ contains the only point of $S$ with maximal multiplicity or $w(H)=3 d$, and $m_{1}=d$. In the latter case we have $h^{1}\left(\mathcal{I}_{Z}(d)\right)=0$ by Lemma 3 .

(b) Assume $w(H)=3 d-1, w(N)=2 d+1, m_{1}=d-1$ and that $N \cap H \cap S=\{o\}$ with $o$ the only point with multiplicity $d-1$ of $Z$. In this case we have $S \subset H \cup N$. Fix $p \in H \cap S \backslash N \cap S$ and let $M$ be the hyperplane spanned by $N$ and $p$. We check for which choice of $p$ the scheme $\operatorname{Res}_{M}(Z)$ satisfies the Segre condition in degree $d-1$. Since $\sharp(S \cap N) \geq 3$ (Remark 2) we have $w_{\operatorname{Res}_{M}(Z)}\left(\mathbb{P}^{4}\right) \leq 4 d+1-4$. Since $p \in H \cap S$ and $w(H)=3 d-1$, $H$ satisfies the Segre condition in degree $d-1$ for $\operatorname{Res}_{M}(Z)$. Fix another $F \in B_{3}$ (if any). Since $w(H)=3 d-1$, the maximality property of $w(H)$ gives $w(F)=3 d-1$. Since $F \neq H, F \cap H$ is a plane and hence $w(F \cap H) \leq 2 d+1$. Hence $w(F \backslash F \cap H) \geq d-2>0$. Since $S \subset H \cup N$, we get $F \cap N \cap S \neq \emptyset$ and hence $w_{\operatorname{Res}_{M}(Z)}(F) \leq 3(d-1)+1$. Fix $A \in B_{2}$. Since $S \subset H \cup N$, we have $A \cap S=(H \cap A \cap S) \cup(N \cap S)$; if $A \nsubseteq H$ we get $w(A \cap H) \leq d+1$ and hence either $A=N$ or $w(A)=2 d$ and $o \in A$; in these $\operatorname{cases}_{\operatorname{Res}_{M}(Z)}(A) \leq 2 d-1$ and hence $A$ satisfies the Segre condition for $\operatorname{Res}_{M}(Z)$. Fix $L \in B_{1}$ and assume $L \cap S \cap N=\emptyset$. Since $S \subset H \cup N$ and $\sharp(L \cap S) \geq 2$, we get $L \subset H$; if it exists we need to take $p \in L$. If the line $L$ is unique any $p \in L \cap S$ will do. Take $R \in B_{1}$ such that $R \cap N \cap S=\emptyset$ and $R \neq L$. Since $w\left(\mathbb{P}^{4}\right) \leq 4 d+1$, we get 
$w(L \cap R) \geq 2(d+1)+(2 d+1)-4 d-1$ and hence $L \cap R \in S$. Since $L \cap R \neq o$, we have $m_{L \cap R} \leq 2$ and hence $w(L \cup R) \geq 2 d$. Since $w\left(\mathbb{P}^{4}\right) \leq 4 d+1$, we get $S \cup L \cup R \cup N$; in this case we tmay $p=L \cap R$, unless there is $D \in B_{1}$ with $D \cap N \cap S=\emptyset$ and $L \cap R \notin D ; D$ is contained in the plane $\langle D \cup R\rangle$ and we have $2 d+1 \geq w(\langle D \cup R\rangle) \geq 3(d+1)-m_{D \cap L}-m_{D \cap R}-m_{R \cap L}$ with $m_{u} \leq 2$ if $u \in\{D \cap L, D \cap R, R \cap L\}$, a contradiction.

\section{5. $B_{2}$}

In this section we assume $B_{3}=\emptyset$ and $m_{1}+m_{2} \leq d$ with the next lemma as the target of the section.

Lemma 7. If $d \geq 5$, then $\sharp\left(B_{2}\right) \leq 1$.

Remark 9. Assume $B_{3}=\emptyset$. If $E \in B_{2}, w(B) \geq d-1$ and $E \cap B \neq \emptyset$, then $E \cap B \cap S \neq \emptyset$. If $A \in B_{2}^{\prime}, B \in B_{1}$ and $A \cap B \neq \emptyset$, then $A \cap B \cap S \neq \emptyset$.

Proof of Lemma \%. Assume $\sharp\left(B_{2}\right) \geq 2$. Call $E, F$ two different elements of $B_{2}$.

Observation 1: Since $B_{3}=\emptyset$ and $w(L) \leq d+1$ for each line $L \subset \mathbb{P}^{4}$, $E \cap F$ is a single point $o$.

Observation 2: If $o \notin S$, then $w\left(\mathbb{P}^{4} \backslash E \cup F\right) \leq 1$ and either $S \subset E \cup F$ or $w(E)=w(F)=2 d$ and $S \cap(E \cup F)$ is a unique point with multiplicity 1, because $w(E) \geq 2 d, w(F) \geq 2 d$ and $w\left(\mathbb{P}^{4}\right) \leq 4 d+1$.

Claim 1: We have $\sharp\left(B_{2}\right) \leq 2$.

Proof of Claim 1: Assume $\sharp\left(B_{2}\right) \geq 3$ and call $E_{1}, E_{2}, E_{3} 3$ distinct elements of $B_{2}$. Observation 1 gives that for each $i \neq j$, the set $E_{i} \cap E_{j}$ is a single point, $o_{h}$, where $\{i, j, h\}=\{1,2,3\}$. With no loss of generality we may assume $m_{o_{1}} \geq m_{o_{2}} \geq m_{o_{3}}$. The points $o_{1}, o_{2}, o_{3}$ are not necessarily distinct, but in any case we have $w\left(E_{1} \cup E_{2} \cup E_{3}\right) \leq w\left(E_{1}\right)+w\left(E_{2}\right)+w\left(E_{3}\right)-m_{o_{1}}-\epsilon_{1} m_{o_{2}}-\epsilon_{2} m_{o_{3}}$, with $\epsilon_{1}=1$ if $o_{1} \neq o_{2}, \epsilon_{2}=1$ if $o_{1}, o_{2}, o_{3}$ are distinct points, $\epsilon_{1}=0$ if $o_{1}=o_{2}$ and $\epsilon_{2}=0$ if $\sharp\left(\left\{o_{1}, o_{2}, o_{3}\right\}\right) \leq 2$. We first get that $\sharp\left(\left\{o_{1}, o_{2}, o_{e}\right\}\right)=3$, and then, using $m_{o_{1}}+m_{o_{3}} \leq m_{o_{1}}+m_{o_{2}} \leq d+1$ and $d \geq 4$, a contradiction.

(a) In this step we assume the existence of $E_{1}, E_{2} \in B_{2}$ such that $E_{1} \cap E_{2} \notin$ $S$. We assume $w\left(E_{1}\right) \geq w\left(E_{2}\right)$.

(a1) Assume $S \subset E_{1} \cup E_{2}$. Fix general hyperplanes $H, H^{\prime}$ containing $E_{1}$ and general hyperplanes $M, M^{\prime}$ containing $E_{2}$. Set $Q:=H \cup M$ and $T:=$ $Q \cap\left(H^{\prime} \cup H^{\prime}\right)$. We first check that $\operatorname{Res}_{Q}(Z)$ satisfies the Segre conditions in degree $d-2$. By [2] we may assume $s \geq 8$ and hence (since $S \subset Q$ ) $\mathbb{P}^{4}$ satisfies the Segre condition for $\operatorname{Res}_{H \cup M}(Z)$. For each $A \in B_{i}^{\prime}, i=1,2,3$, it is sufficient to use that $A \cap S$ spans $A$ (Remark 2). If $A \in B_{2}$ we use then $A=E_{i}$ and we 
use that $\sharp(A \cap S) \geq 4$ (Remark 2). If $A \in B_{1}$, then we use that $\sharp(B \cap S) \geq 2$. By the residual sequence of $Q$ it is sufficient to prove that $h^{1}\left(Q, \mathcal{I}_{Q \cap Z, Q}(d)\right)=$ 0 . The scheme $T$ is an effective Cartier divisor of $Q$ and hence it is defined the residual scheme $\operatorname{Res}_{T}(Z \cap Q)$. Since $\operatorname{Res}_{T}(Z \cap Q) \subseteq \operatorname{Res}_{Q}(Z)$, we have $h^{1}\left(Q, \mathcal{I}_{\operatorname{Res}_{T}(Z \cap Q)}(d-2)\right) \leq h^{1}\left(\mathcal{I}_{\operatorname{Res}_{Q}(Z)}(d-2)\right)=0$. By the residual exact sequence of $T$ in $Q$ it is sufficient to prove that $h^{1}\left(T, \mathcal{I}_{Z \cap T, T}(d)\right)=0$. We have $Z \cap T=Z \cap\left(E_{1} \cup E_{2}\right)$, because $E_{1} \cap E_{2} \notin S$. Use that $h^{1}\left(E_{i}, \mathcal{I}_{Z \cap E_{i}}(d)\right)=0$ by the Segre conditions and that the restriction map $H^{0}\left(\mathcal{O}_{\mathbb{P}^{4}}(d)\right) \rightarrow H^{0}\left(E_{1} \cup\right.$ $\left.E_{2}, \mathcal{O}_{E_{1} \cup E_{2}}(d)\right)$ is surjective (use that $h^{1}\left(\mathbb{P}^{3}, \mathcal{I}_{D \cup E}(2)\right)=0$ for any two skew lines $D, R$ of $\left.\mathbb{P}^{3}\right)$.

(a2) Assume $S \nsubseteq E_{1} \cup E_{2}$. We have $w\left(E_{1}\right)=w\left(E_{2}\right)=2 d, S \backslash S \cap$ $\left(E_{1} \cup E_{2}\right)$ is a single point $o$ and $m_{o}=1$. Let $H_{i}$ be a general hyperplane containing $E_{i}$. Set $Q:=H_{1} \cup E_{2}$. Since $o \notin Q$, the inductive assumption gives $h^{1}\left(Q, \mathcal{I}_{Q \cap Z}(d-2)\right)=0$. Hence it is sufficient to prove that $\operatorname{Res}_{Q}(Z)$ satisfies the Segre conditions in degree $d-2$. Since $\sharp(Q \cap S)=\sharp\left(S \cap E_{1}\right)+\sharp\left(S \cap E_{1}\right) \geq 4+4$, $\mathbb{P}^{4}$ satisfies the Segre condition. Fix $U \in B_{3}^{\prime} \cup B_{3}$. Since $B_{3}=\emptyset$, it is sufficient to prove that $\sharp(U \cap(S \backslash\{o\})) \geq 3$; this is true, because $m_{1}+m_{2} \leq d+1$ and $w(U)-1 \geq 3 d-5>d+1$. Fix $A \in B_{2}^{\prime} \cup B_{2}$. First assume $A \in B_{2}$, i.e. $A=E_{i}$ for some $i$. Since $w\left(E_{i}\right)=2 d$ and $m_{o}=1$, it is sufficient to use that $w\left(E_{i} \cap S\right)-1>m_{1}+m_{2}+m_{3}$; this is true if $2 d-2 \leq m_{1}+m_{2}+m_{3}$, which is true, because $m_{1}+m_{2} \leq d$ and hence $m_{1}+m_{2}+m_{3} \leq 2 d-m_{1}$, while $2 d-2 \geq m_{1}+m_{2}+m_{3}$ if $m_{1}=1$. Now assume $A \in B_{2}^{\prime}$. Use that $w(A)-1>0$ if $w(A)=2 d-2$ and $\sharp\left(A \cap(S \backslash\{o\}) \geq 2\right.$ if $w(A)=2 d-1$ (since $m_{1} \leq 2 d-3$ for all $d \geq 4)$. Fix $B \in B_{1}^{\prime} \cup B_{1}$. If $w(B)=d$ it is sufficient to use that $d>1=m_{o}$. If $w(B)=d+1$ it is sufficient to use that $m_{1}<d$ (Lemma 3).

(b) In this step and in step (c) $\sharp\left(B_{2}\right)=2$, say $B_{2}=\left\{E_{1}, E_{2}\right\}$ and that $\{o\}:=E_{1} \cap E_{2} \in S$. In this step we assume $S \subset E_{1} \cup E_{2}$. Since $\sharp(B \cap S) \geq 3$ for all $B \in B_{1}$, then each element of $B_{1}$ is contained either in $E_{1}$ or in $E_{2}$. Let $\mathcal{T}_{i}, i=1,2$, be the set of all $B \in B_{1}$ such that $B \subset E_{1}$ and $o \notin B$. Assume for the moment $\sharp\left(\mathcal{T}_{i}\right) \leq 2$ for some $i$, say $\sharp\left(\mathcal{T}_{2}\right) \leq 2$. Therefore there is $p \in E_{2} \cap S \backslash\{o\}$ such that every element of $\mathcal{T}_{2}$ contains $p$. Set $H:=\left\langle E_{1} \cup\{p\}\right\rangle$. By construction $\operatorname{Res}_{H}(Z)$ satisfies the Segre conditions in degree $d-1$ with respect to lines and $\mathbb{P}^{4}$, trivially with respect to hyperplanes (because $B_{3}=\emptyset$ ). Fix $A \in B_{2}$, i.e. $A=E_{1}$ or $A=E_{2}$. If $A=E_{1}$, then use that $\sharp(A \cap S) \geq 2$. If $A=E_{2}$, then we use that $o, p \in A \cap H \cap S$. Now assume $\sharp\left(\mathcal{T}_{i}\right) \geq 3$ for all $i$. Fix $D_{1}, D_{2}, D_{3} \in \mathcal{T}_{1}$. By Remark $3 D_{i} \cap D_{j}, i<j$, is an element of $S$. Since $w\left(E_{1}\right) \leq 3(d+1)-m_{D_{i} \cap D_{j}}$ we get that $D_{1} \cap D_{2}, D_{1} \cap D_{3}$ and $D_{2} \cap D_{3}$ are distinct points and $3 d+3-2 d-1+m_{o} \leq m_{D_{1} \cap D_{2}}+m_{D_{1} \cap D_{3}}+m_{D_{2} \cap D_{3}}$, i.e.

$$
d+2+m_{o} \leq m_{D_{1} \cap D_{2}}+m_{D_{1} \cap D_{3}}+m_{D_{2} \cap D_{3}}
$$


Moreover, no 3 of the points $o, D_{1} \cap D_{2}, D_{1} \cap D_{3}$ and $D_{2} \cup D_{3}$ are collinear. Hence $\mathcal{I}_{\left\{D_{1} \cap D_{2}, D_{1} \cap D_{3}, D_{3} \cap D_{3}\right\} \cup E_{2}}(2)$ is spanned. Thus there is a quadric hypersurface $Q \in\left|\mathcal{I}_{\left\{D_{1} \cap D_{2}, D_{1} \cap D_{3}, D_{3} \cap D_{3}\right\} \cup E_{2}}(2)\right|$ with $Q \cap S \neq S$. By the inductive assumption it is sufficient to prove that $\operatorname{Res}_{Q}(Z)$ satisfies the Segre conditions in degree $d-2$.

(b1) These conditions are satisfied by $\mathbb{P}^{4}$, each element of $B_{2}$, each element of $B_{1} \cup B_{1}^{\prime}$ contained in $E_{2}$. They are satisfied by each $D_{i}$ and each $B \in B_{1} \backslash$ $\left\{D_{1}, D_{2}, D_{3}\right\}$ contained in $E_{1}$, because $B \cap\left(D_{1} \cup D_{2} \cup D_{3}\right)=S \cap B \cap\left(D_{1} \cup D_{2} \cup D_{3}\right)$ (Remark 3) and $\sharp\left(B \cap\left(D_{1} \cup D_{2} \cup D_{3}\right)\right) \geq 2$, since $D_{1} \cap D_{2} \cap D_{3}=\emptyset$. They are satisfied by each $B \in B_{1}^{\prime}$ contained in $E_{1}$, because $w\left(E_{1} \backslash\left\{o, D_{1} \cap D_{2}, D_{1} \cap\right.\right.$ $\left.\left.D_{3}, D_{3} \cap D_{3}\right\}\right)<d$ by (3), and all other elements of $B_{1}^{\prime}$ meets $E_{2} \cap S$, since $S \subset E_{1} \cup E_{2}$.

(b2) Fix $U \in B_{3}^{\prime}$. We have $\sharp(S \cap Q \cap U) \geq w(U)-3 d+5$, because $w\left(\mathbb{P}^{4} \backslash Q\right) \leq 4 d+1-2 d-3(d+1)+m_{D_{1} \cap D_{2}}+m_{D_{1} \cap D_{3}}+m_{D_{2} \cap D_{3}}=m_{D_{1} \cap D_{2}}+$ $m_{D_{1} \cap D_{3}}+m_{D_{2} \cap D_{3}}-d-2 \leq d / 2$.

(b3) Fix $A \in B_{2}^{\prime}$. First assume $o \notin A$. To check the Segre condition for $A$ we may assume $\sharp\left(A \cap E_{1} \cap S\right) \leq 1$. Since $A \cap S$ spans $A$ we get that $A$ contains a unique point $E_{2} \cap S \backslash\{o\}$, while the other points are contained in a line $L \subset E_{1}$; we get $w(L) \geq w(A)-m_{q}$; to check the Segre for $A$ we may assume $w(A)=2 d-1$ and in this case we get $L \in B_{1}^{\prime}$ and we checked that $L \cap Q \cap S \neq \emptyset$. Now assume $o \in A$. To check the Segre condition for $A$ we may assume $w(A)=2 d-1$ and that $A \cap E_{2} \cap S=\{o\}$. Since $S \subset E_{1} \cup E_{2}$ and $A \cap S$ spans $A$ (Remark 2), we get $A \subseteq E_{1}$, a contradiction.

(c) Assume $o \in S$ and $S \nsubseteq E_{1} \cup E_{2}$. We have $w\left(\mathbb{P}^{4} \backslash\left(E_{1} \cup E_{2}\right)\right) \leq$ $4 d+1-2 d-2 d+m_{o}=m_{o}+1$ and equality holds only if $w\left(\mathbb{P}^{4}\right)=4 d+1$ and $w\left(E_{i}\right)=2 d$ for all $i$.

Since $\mathcal{I}_{E_{1} \cup E_{2}}(2)$ is spanned (use reducible quadrics) there is $Q \in\left|\mathcal{I}_{E_{1} \cup E_{2}}(2)\right|$ such that $Q \cap S \neq S$. The inductive assumption on the weight gives $h^{1}\left(\mathcal{I}_{Z \cap Q}(2)\right)=$ 0 and so it is sufficient to prove that $\operatorname{Res}_{Q}(Z)$ satisfies the Segre conditions in degree $d-2$. We will find some obstructions and take a suitable $q$ to overcome them. Since $Q \supset E_{1} \cup E_{2}$ and $\sharp\left(S \cap E_{i}\right) \geq 4, \mathbb{P}^{4}, E_{1}$ and $E_{2}$ satisfy the Segre condition. Fix $U \in B_{3} \cup B_{3}^{\prime}$. Since $w\left(\mathbb{P}^{4} \backslash Q\right) \leq w\left(\mathbb{P}^{4} \backslash\left(E_{1} \cup E_{2}\right)\right) \leq m_{o}+1$, we get $S \cap U \cap Q \neq \emptyset$. If $w(U) \geq 3 d-3$, we also get $\sharp(U \cap Q \cap S) \geq 2$, because $1+2 m_{2} \leq 2 d-1 \leq 3 d-4$. Now assume $w(U)=3 d-2$ and $\sharp(S \cap U \cap Q)=2$, say $S \cap U \cap Q=\{p, q\}$. We get $m_{p}+m_{q}+m_{o}+1 \geq 3 d-2$; if $o \in\{p, q\}$, then we use that $2 m_{1}+m_{2} \leq 3 d-4$, since $m_{1} \leq d-1$ (Lemma 3), $m_{1}+m_{2} \leq d$ and $d \geq 5$; if $o \notin\left\{p_{q}\right\}$ we use that $m_{1}+m_{2}+m_{3}+1 \leq 2 d+1-m_{1}+m_{3}$.

Take $B \in B_{1} \cup B_{1}^{\prime}$. If $B \cap Q \cap S=\emptyset$, the $w(B) \leq 1+m_{o}$; we get $w(B)=d$ and $m_{o}=d-1$; we also get $w\left(\mathbb{P}^{4}\right)=4 d+1$ and $w\left(E_{i}\right)=2$ for all $i$; call + this case. Take $B \in B_{1}$ with $\sharp(B \cap Q \cap S)=1$, say $B \cap Q \cap S=\{q\}$. First 
assume $q=o$; we also get $w\left(\mathbb{P}^{4}\right)=4 d+1$ and $w\left(E_{i}\right)=2$ for all $i$ in this case $w_{\operatorname{Res}_{Q}(Z)}(B)=d-1$, because $Q$ is singular at $o$. Now assume $q \neq o$. We get $w\left(\mathbb{P}^{4}\right)=4 d+1$ and $m_{q}+m_{o}=d$; call ++ this case. Take $A \in B_{2}^{\prime}$. Since $w\left(\mathbb{P}^{4} \backslash\left(E_{1} \cup E_{2}\right)\right) \leq m_{o}+1 \leq d<2 d-2$, we have $\sharp(A \cap Q \cap S) \neq \emptyset$. Assume $w(A)=2 d-1$ and $\sharp(A \cap Q \cap S)=1$, say $A \cap Q \cap S=\{q\}$. We get $m_{q}+m_{o}+1 \leq 2 d-1$; this is possible only if $q=o$ and in this case $w_{\operatorname{Res}_{Q}(Z)}(A) \leq 2 d-4$, because $Q$ is singular at $o$. In summary, for any $Q$ the Segre conditions are satisfied by all linear spaces of dimension $>1$. Hence from now on we assume $w\left(\mathbb{P}^{4}\right)=4 d+1$ and $w\left(E_{i}\right)=2 d, i=1,2$.

(c1) Assume case + , i.e. $m_{o}=d-1$. Since $m_{1}+m_{2} \leq d$, we get $m_{i}=1$ for all $i>0$. We saw the existence of $B \in B_{1}^{\prime}$ such that $S \subset E_{1} \cup E_{2} \cup B$ and $B \cap\left(E_{1} \cup E_{2}\right) \cap S=\emptyset$ and that for an arbitrary $Q B$ is the only linear space for which the Segre condition in degree $d-2$ may fail. By Remark 9 we have $B \cap E_{=} \emptyset$. Fix $p \in B \cap S$ and set $M:=\left\langle E_{1} \cup\{p\}\right\rangle$. Let $H$ be any hyperplane with $H \supset E_{2}$ and $H \cap B \cap S=\emptyset$. The latter condition gives $(M \cup H) \cap S \neq \emptyset$. The former condition gives that $\operatorname{Res}_{H \cup M}(Z)$ satisfies the Segre condition for $B$ and hence for all linear spaces.

(c2) Assume case ++ , i.e. the existence of $L \in B_{1}$ and $q \in\left(E_{1} \cup E_{2}\right) \cap$ $S \backslash\{o\}$ with $m_{q}+m_{o}=d$ and $L \cap\left(E_{1} \cap E_{2}\right) \cap S=\{q\}$. We also have $S \subset L \cup E_{1} \cup E_{2}$ and that $L$ is the only linear space for which (for an arbitrary $\left.Q \supset E_{1} \cup E_{2}\right)$ the Segre condition in degree $d-2$ may fail. With no loss of generality we may assume $q \in E_{1}$. Set $H:=\left\langle E_{2} \cup\{q\}\right\rangle$ and $D:=\langle\{o, q\}\rangle \in B_{1}^{\prime}$. $\operatorname{Res}_{H}(Z)$ satisfies the Segre conditions in degree $d-1$ with respect to $\mathbb{P}^{4}$ (because $\sharp(H \cap S) \geq 4)$ and with respect to all hyperplanes. Fix $R \in B_{1}$. If $R \cap E_{2} \cap S \neq \emptyset$, then $w_{\operatorname{Res}_{H}(Z)}(R) \leq d$. If $R \cap E_{2} \cap S=\emptyset$, then either $R=L$ or $R \subset E_{1}$, because $S \subset L \cup E_{1} \cup E_{2}$ and $\sharp(R \cap S) \geq 3$. If $R=L$, then we use that $q \in H$. If $R \subset E_{1}$, then $D \cap R \cap S \neq \emptyset$, because $D \subset E_{1}, R \subset E_{1}$ and $w(D)+w(R)=2 d+1>w\left(E_{1}\right)$.

\section{3 Mutually Disjoint Elements of $B_{1}$}

In this section we assume $B_{3} \neq \emptyset$ and the existence of $L_{1}, L_{2}, L_{3} \in B_{1}$ such that $L_{i} \cap L_{j}=\emptyset$ for all $i \neq j$. With this assumption we prove that $h^{1}\left(\mathcal{I}_{Z}(d)\right)=0$.

By Remark 7 we have $\left(L_{1} \cup L_{2} \cup L_{3}\right) \cap S \cap R \neq \emptyset$ for all $R \in B_{1} \cup B_{1}^{\prime}$. We have $w\left(\mathbb{P}^{4} \backslash\left(L_{1} \cup L_{2} \cup L_{3}\right)\right) \leq 4 d+1-3(d+1)=d-2$. Let $J$ be the only line meeting all lines $L_{1}, L_{2}, L_{3}$ (Remark 8).

We often use the notation introduced in the statement of the following lemma. 
Lemma 8. For any $i, j \in\{1,2,3\}$ with $i \neq j$ let $U_{h}, h:=\{1,2,3\} \backslash\{i, j\}$, be the hyperplane spanned by $L_{i} \cup L_{j}$ and $F_{i}$ the plane spanned by $L_{i} \cup J$. Take $a \in \mathbb{P}^{4} \backslash J \cup L_{1} \cup L_{2} \cup L_{3}$. If $a \in F_{i}$ for some $i$, then $\left|\mathcal{I}_{\{a\} \cup J \cup L_{1} \cup L_{2} \cup L_{3}}(2)\right|$ has $F_{i} \cup L_{1} \cup L_{2} \cup L_{3}$ as its base locus. In all other cases, the base locus is $\{a\} \cup J \cup L_{1} \cup L_{2} \cup L_{3}$.

Proof. First assume that $a \in U_{h}$ for some $U_{h}$, say $h=1$. Take reducible quadrics $U_{1}$ as a component and use that $\mathcal{I}_{J \cup L_{i} \cup L_{j} \cup\{a\}, U_{1}}(2)$ has no base points outside $U_{1} \cap\left(J \cup L_{1} \cup L_{2} \cup L_{3} \cup\{a\}\right)$, unless $a \in F_{2} \cup F_{3}$, say $a \in F_{3}$. Using hyperplanes containing $L_{3}$ we see that the same is true for $\left|\mathcal{I}_{\{a\} \cup J \cup L_{1} \cup L_{2} \cup L_{3}}(2)\right|$. Now assume $a \notin U_{h}$ for any $h$. The linear system of hyperplanes containing $L_{h}$ and $a$ has the plane $E_{h, a}:=\left\langle L_{h} \cup\{a\}\right\rangle$ as its base locus. The family of reducible quadrics $U_{h} \cup M, M$ a hyperplane containing $E_{h, a}$, shows that outside $J \cup L_{1} \cup L_{2} \cup L_{3}$ the base locus is contained in $E_{h, a}$. Since $E_{1, a} \cap E_{2, a} \cap E_{3, a}=\{a\}$, we get the lemma even in this case.

Lemma 9. Fix a plane $A \subset \mathbb{P}^{4}$ and lines $L_{i} \subset \mathbb{P}^{4}, 1 \leq i \leq 3$, such that $L_{i} \cap L_{j}=\emptyset$ for all $i \neq j, \sharp\left(A \cap L_{i}\right)=1$ for all $i$ and $A$ not the intersection of two hyperplanes $\left\langle L_{i} \cup L_{j}\right\rangle$, i.e. $A \neq F_{h}$ for any $h$. Then $h^{0}\left(\mathcal{I}_{A \cup L_{1} \cup L_{2} \cup L_{3}}(2)\right)=3$.

Proof. By assumption $A$ is contained in at most one hyperplane $\left\langle L_{i} \cup\right.$ $\left.L_{j}\right\rangle$. Hence may assume that $A \nsubseteq\left\langle L_{1} \cup L_{3}\right\rangle$ and that $A \nsubseteq\left\langle L_{2} \cup L_{3}\right\rangle$. Set $\left\{o_{i}\right\}:=A \cap L_{i}$. Since each $L_{i}$ meets $A$, we have $h^{0}\left(\mathcal{I}_{A \cup L_{1} \cup L_{2} \cup L_{3}}(2)\right) \geq 3$. We have $h^{1}\left(\mathcal{I}_{A \cup L_{1} \cup L_{2}}(2)\right)=0$ (all triples $\left(A, L_{1}, L_{2}\right)$ are projectively equivalent), i.e $h^{0}\left(\mathcal{I}_{A \cup L_{1} \cup L_{2}}(2)\right)=5$. Since $A \nsubseteq\left\langle L_{h} \cup L_{3}\right\rangle$ for any $h \in\{1,2\}$, the set $\left\langle A \cup L_{h}\right\rangle \cap L_{3}, h=1,2$, is a unique point, $e_{h}$. Fix $p \in L_{3}$ with $p \neq o_{3}$ and $p \neq e_{1}$. Taking the union of $\left\langle A \cup L_{1}\right\rangle$ and a general hyperplane $M$ containing $L_{2}$ we get $h^{0}\left(\mathcal{I}_{A \cup L_{1} \cup L_{2} \cup\{p\}}(2)\right)=4$. Let $H$ be a general hyperplane containing $L_{1} \cup\{p\}$. Fix $o \in L_{3} \backslash\left\{p, e_{1}, e_{2}\right\}$. Since $H \cup\left\langle A \cup L_{2}\right\rangle \in\left|\mathcal{I}_{A \cup L_{1} \cup L_{2} \cup\{p\}}(2)\right|$ and $o \notin H \cup\left\langle A \cup L_{2}\right\rangle$, we get $h^{0}\left(\mathcal{I}_{A \cup L_{1} \cup L_{2} \cup L_{3}}(2)\right) \leq 3$.

Lemma 10. Assume $S \subset L_{1} \cup L_{2} \cup L_{3}$. Then $h^{1}\left(\mathcal{I}_{Z}(d)\right)=0$.

Proof. Fix $N \in B_{2}$. Since $\sharp(N \cap S) \geq 4$, it contains at least one of the lines $L_{i}$. Since $N$ is unique (Lemma 7), we may change the labels, so that, if $N$ exists, then it contains $L_{1}$. Since $\sharp(B \cap S) \geq 3$ for all $B \in B_{1}$ (Remark $6)$ ), we have $B_{1} \subseteq\left\{L_{1}, L_{2}, L_{3}, J\right\}$. Fix a hyperplane containing $L_{1}$, a point of $L_{2} \cap S$ and a point of $L_{3} \cap S$. Fix $B \in B_{1}$. Since $\sharp(B \cap S) \geq 3$, either $B \cap S$ meets each set $L_{i} \cap S, i=1,2,3$, or $B$ contains at least two points of some $L_{i}$ and so $B=L_{i}$. In both cases we have $B \cap S \cap H \neq \emptyset$. The scheme $\operatorname{Res}_{H}(Z)$ 
satisfies the Segre condition in degree $d-1$ with respect to $\mathbb{P}^{4}$, all lines, all planes (because $N \supset L_{1}$ ) and (since $B_{3}=\emptyset$ ) all hyperplanes.

Lemma 11. If $S \subset J \cup L_{1} \cup L_{2} \cup L_{3}$, then $h^{1}\left(\mathcal{I}_{Z}(d)\right)=0$.

Proof. By Lemma 10 we may assume $S \nsubseteq L_{1} \cup L_{2} \cup L_{3}$. By Remark 6 we have $B_{1} \subseteq\left\{L_{1}, L_{2}, L_{3}, J\right\}$.

(i) First assume $J \cap S \cap\left(L_{1} \cup L_{2} \cup L_{3}\right) \neq \emptyset$, say $J \cap L_{3} \in S$. Let $H$ be a hyperplane containing $F_{1}$ and a point of $L_{2} \cap S \backslash J \cap S$ with maximal multiplicity. It is sufficient to prove that $\operatorname{Res}_{H}(Z)$ satisfies the Segre conditions in degree $d-1$. It satisfies the Segre condition for $\mathbb{P}^{4}$, because $\sharp\left(L_{1} \cap S\right) \geq 3$. It satisfies the Segre condition for lines, because $H \cap S$ meets $J$ and each $L_{i}$. Since $B_{3}=\emptyset$ by assumption, we only need to check the Segre condition for planes. Fix $N \in B_{2}$. We have $\sharp(N \cap S) \geq 4$ (and hence either $N$ contains a different point of $J \cap S, L_{1} \cap S, L_{2} \cap S, L_{3} \cap S$ or $N$ contains a line $L_{i}$ ) with equality only if $w(N)=2 d$ (Remark 6). Therefore $N \cap S \cap H \neq \emptyset$ and to check the Segre condition for $\operatorname{Res}_{H}(Z)$ it is sufficient to check the case $w(N)=2 d+1$ with $N$ containing one among $L_{2}$ and $L_{3}$ (say $L_{h}$ ) but not the other one. If $N=F_{h}$, we get $\sharp(S \cap N \cap H) \geq 2$, because $J \subset H$. Now assume $N \neq F_{h}$ and so $N \cap J=L_{h} \cap J$. Since $w\left(N \backslash L_{h}\right)=d$, we have $\sharp\left(N \cap S \backslash L_{h} \cap S\right) \geq 2$ and these points must be contained in $L_{1} \cup L_{5-h}$, no two of them in the same line (since two of the lines $L_{1}, L_{2}, L_{3}$ spans a $\left.\mathbb{P}^{3}\right)$. Hence $\sharp(N \cap S \cap H) \geq 2$.

(ii) Now assume $J \cap S \cap\left(L_{1} \cup L_{2} \cup L_{3}\right)=\emptyset$. Hence $w(J) \leq d-2$, $B_{1}=\left\{L_{1}, L_{2}, L_{3}\right\}, B_{1}^{\prime}=\emptyset$ and $w\left(F_{i}\right) \leq 2 d-1$.

Take a hyperplane $U$ containing one of the points with maximal multiplicity of each line $J, L_{1}, L_{2}, L_{3}$ and spanned by points of $S$, except that if $B_{2} \neq \emptyset$, say $B_{2}=\{N\}$ (Lemma 7), we impose that for any line $J, L_{1}, L_{2}, L_{3}$ intersecting $N \cap S$ we impose that we take this point as one of the prescribed points of $U \cap S$. We need to check the Segre conditions for $\operatorname{Res}_{U}(Z)$ in degree $d-1$. The one for $\mathbb{P}^{4}$ is satisfied, because $\sharp(U \cap S) \geq 4$ by its definition. The Segre conditions for lines are satisfied, because $L_{i} \cap S \cap U \neq \emptyset$. Since $B_{3}=\emptyset$, it is sufficient to check the ones for planes $N \in B_{2}$. By Remark ?? $S \cap(N \backslash J)$ contains at least two points of $L_{1} \cup L_{2} \cup L_{3}$, because $w\left(F_{i}\right) \leq 2 d-1$ and hence $N \neq F_{i}$.

Remark 10. Fix a quadric $Q \supset L_{1} \cup L_{2} \cup L_{3}$ and assume $Q \cap S \neq S$, so that the inductive assumption gives $h^{1}\left(\mathcal{I}_{Z \cap Q}(d)\right)=0$. We check what conditions on $Z$ and $Q$ gives that $\operatorname{Res}_{Q}(Z)$ satisfies the Segre condition in degree $d-2$. The condition for $\mathbb{P}^{4}$ is satisfied, because $\sharp\left(L_{i} \cap S\right) \geq 3$. Fix $U \in B_{3}^{\prime} \cup B_{3}=B_{3}^{\prime}$. Since $w\left(\mathbb{P}^{4} \backslash\left(L_{1} \cup L_{2} \cup L_{3}\right)\right) \leq d-2$ and $d-2+m_{1} \leq 2 d-3<3 d-4$, we have $U \cap Q \cap S \neq \emptyset$. If $w(U) \geq 3 d-3$ (resp. $w(U)=3 d-2$ ), then we have 
$\sharp(U \cap Q \cap S) \geq 2$ (resp. $\sharp(U \cap Q \cap S) \geq 3$ ), because $d-2+m_{1}<3 d-3$ (resp. $d-2+m_{1}+m_{2} \leq 2 d-2<3 d-2$ ). Fix $A \in B_{2} \cup B_{2}^{\prime}$. Since $d-2<2 d-2$ and $d-2+m_{1}<2 d-1$, we have $A \cap Q \cap S \neq \emptyset$ and $\sharp(A \cap Q \cap S) \geq 2$ if $w(A) \geq 2 d-1$. If $w(A) \geq 2 d$, then $\sharp(A \cap H \cap S) \geq 3$, because $d-2+m_{1}+m_{2}<w(N)$. Thus we only need to test the element of $B_{2}$ if $B_{2} \neq \emptyset$ and only if it has weight $2 d+1$. Fix $B \in B_{1}^{\prime}$. By Remark 5 we have $S \cap B \cap\left(L_{1} \cup L_{2} \cup L_{2}\right) \neq \emptyset$ and hence $w_{\operatorname{Res}_{Q}(Z)}(B) \leq d+1$. Let $\mathcal{B}$ be the set of all $B \in B_{1}$ such that $\sharp\left(S \cap B \cap\left(L_{1} \cup L_{2} \cup L_{3} \cap J\right)\right)=1$. Fix $B \in B_{1}$. By Remark 5 we have $S \cap B \cap\left(L_{1} \cup L_{2} \cup L_{2}\right) \neq \emptyset$. Since $J$ is in the base locus of $\left|\mathcal{I}_{L_{1} \cup L_{2} \cup L_{3}}(2)\right|, B$ may fail the test (for some $Q$ ) only if $B \in \mathcal{B}$. If $B \in \mathcal{B}$, then $B \cap J \cap S \subset\left(L_{1} \cup L_{2} \cup L_{3}\right)$. For $i=1,2,3$ set $\mathcal{B}_{i}:=\left\{L \in \mathcal{B}: L \cap L_{i} \neq \emptyset\right\}$.

Remark 11. Fix a plane $N \in B_{2} \cup B_{2}^{\prime}$ and set $x:=w(N)$. If $(y-$ 1) $\lfloor d / 2\rfloor+d-2<x$, then $\sharp\left(N \cap S \cap\left(L_{1} \cup L_{2} \cup L_{3}\right)\right) \geq y$. If $x \geq 2 d-1$, we may take $y \geq 3$, while if $x=2 d-2$ we have $y>0$. So only the planes $N$ with $w(N)=2 d+1$ may give troubles. Here we assume $w(N)=2 d+1$. By Lemma 8 we may assume $\sharp\left(B_{2}\right)=1$ and we fix $p \in N \cap S \backslash S \cap\left(L_{1} \cup L_{2} \cup L_{3}\right)$. Assume the existence of a plane $w(N)=2 d+1$ and $\sharp\left(N \cap S \cap\left(L_{1} \cup L_{2} \cup L_{3}\right)\right)=3(N$ is unique by Lemma 7). Set $N \cap S \cap\left(L_{1} \cup L_{2} \cup L_{3}\right)=\left\{o_{1}, o_{2}, o_{3}\right\}$ with, say $m_{o_{1}} \geq m_{o_{2}} \geq m_{o_{3}}$. Since $w\left(\mathbb{P}^{4} \backslash\left(L_{1} \cup L_{2} \cup L_{3}\right)\right) \leq d-2$, and $w(N)>d-2+d+1$, the points $o_{1}, o_{2}, o_{3}$ are not collinear. Hence $\sharp\left(S \cap L_{i}\right)=1$ for all $i$ and $N \nsupseteq L_{i}$ for any $i$. In particular $N \neq F_{i}$ for any $i$. We also get that at most one of the points $o_{1}, o_{2}, o_{3}$ is contained in $J$. Since $w\left(\mathbb{P}^{4} \backslash\left(L_{1} \cup L_{2} \cup L_{3}\right)\right) \leq d-2$, we get $m_{o_{1}}+m_{o_{2}}+m_{o_{3}} \geq d+3$. If $N$ contains a point of $J \cap S$ distinct from the points $o_{1}, o_{2}, o_{3}$, then $w_{\operatorname{Res}_{Q}(Z)}(N) \leq 2 n-3$, because $Q \supset J$. We say that $N$ exists only if $N \cap J \cap S \subset\left\{o_{1}, o_{2}, o_{3}\right\}$. Since $m_{1}+m_{2} \leq d$ by our assumption $\diamond \boldsymbol{A}$, we have $m_{1}+m_{2}+m_{3}+m_{4} \leq 2 d<w(N)$. Thus $\sharp(N \cap S) \geq 5$, Assume $\mathcal{B} \neq \emptyset$. Fix any $D \in \mathcal{B}$ and set $\{u\}:=S \cap D \cap\left(L_{1} \cup L_{2} \cup L_{3}\right)=S \cap D \cap\left(J \cup L_{1} \cup L_{2} \cup L_{3}\right)$. Since $m_{1}+m_{2} \leq d$, we have either $m_{o_{1}}+m_{o_{2}}+m_{o_{3}}+m_{u} \leq \min \left\{4 m_{o_{1}}, 3 d-\right.$ $\left.2 m_{o_{1}}\right\}<w(N)+w(D)-d+2\left(\right.$ case $\left.m_{o_{1}} \geq m_{u}\right)$ or $m_{o_{1}}+m_{o_{2}}+m_{o_{3}}+m_{u} \leq$ $\min \left\{4 m_{u}, 3 d-2 m_{u}\right\}<w(N)+w(D)-d+2$ (case $m_{u}>m_{o_{1}}$ ) and hence $D \cap S \cap\left(N \backslash N \cap\left(L_{1} \cup L_{2} \cup L_{3}\right)\right) \neq \emptyset$.

Remark 12. Assume $F_{1} \in B_{2}$. Fix $h \in\{2,3\}$. Since $w\left(\left\langle F_{1} \cup L_{h}\right\rangle\right) \geq$ $2 d+d+1-m_{J \cap L_{h}}$ and $B_{3}=\emptyset$, we have $J \cap L_{h} \in S$ for all $h$. By the definition of existence of $N$ given in Remark 11 if $N$ exists, then $N \neq F_{i}$ for any $i$.

Remark 13. Assume $\sharp(\mathcal{B}) \geq 2$ and $D_{i} \in \mathcal{B}, i=1,2$ with $D_{1} \neq D_{2}$. We have $D_{1} \cap D_{2} \cap\left(L_{1} \cup L_{2} \cup L_{3}\right)=\emptyset$, because $\sharp\left(D_{1} \cap D_{2}\right) \leq 1$ and $2(d+1)>m_{u}+d-2$ for all $u \in \mathbb{P}^{4}$. Set $q_{i}:=D_{i} \cap\left(L_{1} \cup L_{2} \cup L_{3}\right)$. We have $D_{1} \cap D_{2} \cap S \neq \emptyset$ (call $q$ this point), because $m_{q_{1}}+m_{q_{2}}+d-2<2 d+2$. We have $m_{q_{1}}+m_{q_{2}}+m_{q}+d-2 \geq 2 d+2$ 
and hence $m_{q_{1}}+m_{q_{2}}+m_{q} \geq d+4$.

(a) We also get that $q_{1} \neq q_{2}$ and hence that if $\sharp\left(\mathcal{B}_{j}\right) \geq 2$, no two of the lines of $\mathcal{B}_{j}$ contain the same point of $L_{j}$.

Assume the existence of another line $D_{3} \in \mathcal{B}$. Since $D_{i} \cap D_{j} \neq \emptyset$ for all $i, j$, either the lines $D_{1}, D_{2}, D_{3}$ are contained in a plane, $A$, or they pass through the same point. In the latter case $q$ is their common point.

Assume for the moment that $D_{1}$ and $D_{2}$ meets the same line $L_{i}$. Since $D_{1} \cap D_{2} \neq \emptyset, L_{i} \subset\left\langle D_{1} \cup D_{2}\right\rangle$. Hence each $D$ with $q \notin D$ meets $L_{i}$ and hence $\left\langle D_{1} \cup L_{i}\right\rangle$ is the plane $A$ in this case. In the other case in which there is a plane $A$ we have $\sharp(\mathcal{B})=3, \sharp\left(A \cap L_{j}\right)=1$ for all $j$ and we may rename the lines so that $D_{i} \cap L_{j} \neq \emptyset$ if and only if $i=j$. In the former case either $D_{1}, D_{2}, D_{3}$ meets different lines $L_{1}, L_{2}, L_{3}$, say $D_{i} \cap L_{j} \neq \emptyset$ for all $i \neq j$, or they meet the same line $L_{i}$, and $A=\left\langle L_{i} \cup D_{1}\right\rangle$. Hence if all lines $D \in B_{1}$ with $\sharp\left(D \cap\left(L_{1} \cup L_{2} \cup L_{3}\right) \cap S\right)=1$ are through a common point, there are at most 3 of them. If they are all contained in $A$, either all intersects $L_{i}$ and $D_{i}$ (at a point of $S$ by Remark 2) or there are at most 3 such lines and $\sharp\left(A \cap L_{i}\right)=1$ for all $i$ such that $L_{i} \cap D$ for some $i$; in the first case they meets $D_{i}$ and $L_{i}$ at a point $\neq L_{i} \cap D_{1}$ and hence $\sharp\left(D \cap S \cap\left(L_{i} \cup D_{1}\right) \cap S\right) \geq 2$ for all $D \in B_{1}$ with $\sharp\left(D \cap\left(L_{1} \cup L_{2} \cup L_{3}\right) \cap S\right)=1$.

Lemma 12. Assume that $N$ exists and set $\left\{o_{1}, o_{2}, o_{3}\right\}:=N \cap S \cap\left(L_{1} \cup\right.$ $\left.L_{2} \cup L_{3}\right)$ with $o_{i} \in L_{i}$. If $R \in \mathcal{B}_{i}$ and $R \nsubseteq N$, then $o_{i} \notin R$.

Proof. Assume $o_{i} \in R$. Since $\sharp(R \cap N) \leq 1$, we get $3 d+2=w(N)+w(R) \leq$ $d-2+m_{o_{1}}+m_{o_{2}}+m_{o_{3}}$, a contradiction.

Lemma 13. Assume that $N$ exists and that it contains $B \in \mathcal{B}$. Then $h^{1}\left(\mathcal{I}_{Z}(d)\right)=0$.

Proof. With no loss of generality we may assume $B \in \mathcal{B}_{1}$. If $o_{1} \notin B$, then $N=\left\langle B \cup L_{1}\right\rangle$ and hence $N \supset L_{1}$, a contradiction. Since all elements of $\mathcal{B}_{1}$ contain $o_{1}$, part (a) of Remark 13 gives $\mathcal{B}_{1}=\{B\}$. We have $h^{0}\left(\mathcal{I}_{B \cup L_{1} \cup L_{2} \cup L_{3}}(2)\right) \geq$ 4. Let $\tau$ be the base locus of $\left|\mathcal{I}_{B \cup L_{1} \cup L_{2} \cup L_{3}}(2)\right|$. Fix a general $Q \in\left|\mathcal{I}_{B \cup L_{1} \cup L_{2} \cup L_{3}}(2)\right|$. Since $Q \cap S \backslash N \cap S \cap\left(L_{1} \cup L_{2} \cap L_{3}\right) \neq \emptyset$ and each element of $\mathcal{B}$ meets $B \cap S \backslash\left\{o_{1}\right\}$ (Remark 13), Remark 11 gives that $\operatorname{Res}_{Q}(Z)$ satisfies the Segre conditions in degree $d-2$. Hence $h^{1}\left(\mathcal{I}_{Z}(d)\right)=0$, unless $S \subset \tau$. Since $N \neq F_{h}$ for any $h$ (Remark 12), we have $h^{0}\left(\mathcal{I}_{N \cup L_{1} \cup L_{2} \cup L_{3}}(2)\right)=3$ (Lemma 9). Since every quadric hypersurface containing $B \cup L_{1}$ and a point of $N \backslash B \cup L_{1}$ contains $N$, we get $h^{0}\left(\mathcal{I}_{B \cup L_{1} \cup L_{2} \cup L_{3}}(2)\right)=4$. Hence $\tau \cap N=B \cup L_{1}$. Hence $N \cap S=\left(L_{1} \cap S\right) \cup(B \cap S)$. We get $m_{o_{1}}=w\left(L_{1}\right)+w(B)-w(N)=1$. Since $m_{o_{1}}+m_{o_{2}}+m_{o_{3}} \geq d+3$, we get a contradiction. 
Lemma 14. If $\mathcal{B}=\emptyset$, then $h^{1}\left(\mathcal{I}_{Z}(d)\right)=0$.

Proof. By Remarks 10 and 11 we may assume that $N$ exists with $N \cap L_{i}=$ $\left\{o_{i}\right\} \in S, i=1,2,3, o_{1}, o_{2}, o_{3}$, not collinear, and $N \cap J \cap S \subset\left\{o_{1}, o_{2}, o_{3}\right\}$. Fix $o \in N \cap S \backslash\left\{o_{1}, o_{2}, o_{3}\right\}$ and let $\tau$ be the base locus of $\left|\mathcal{I}_{\{o\} \cup L_{1} \cup L_{2} \cup L_{3}}(2)\right|$. Fix a general $Q \in\left|\mathcal{I}_{\{0\} \cup L_{1} \cup L_{2} \cup L_{3}}(2)\right|$. We have $w_{\operatorname{Res}_{Q}(Z)}(N) \leq 2 d-3$. Since $\mathcal{B}=\emptyset$, $\operatorname{Res}_{Q}(Z)$ satisfies the Segre conditions in degree $d-2$. Hence $h^{1}\left(\mathcal{I}_{Z}(d)\right)=0$, unless $S \subset \tau$. If $o \notin F_{i}$ for any $i$, then $\tau=\{o\} \cup J \cup L_{1} \cup L_{2} \cup L_{3}$ (Lemma 8). Since $\sharp(N \cap S) \geq 5$ (Remark 11), we get $S \nsubseteq \tau$, a contradiction. If $o \in F_{i}$ for some $i$, say for $i=1$, then $\tau=F_{1} \cup L_{2} \cup L_{3}$. Hence $S \subset F_{1} \cup L_{2} \cup L_{3}$ and hence $N \cap S=\left(F_{1} \cap N \cap S\right) \cup\left(L_{2} \cap N \cap S\right) \cup\left(L_{3} \cap N \cap S\right)=\left(F_{1} \cap N \cap S\right) \cup\left\{o_{2}, o_{3}\right\}$. Since $N \neq F_{i}$ (Remark 12), $F_{1} \cap N$ is a point or a line. Since $w(N)=2 d+1$, we get that $N \cap F_{1}$ is a line, $N \cap B_{1} \in B_{1}$ and $m_{o_{2}}+m_{o_{3}}=d$. Since $\mathcal{B}=\emptyset$, $F_{1} \nsupseteq L_{2}, F_{1} \nsupseteq L_{3}$ and $J$ is the only line meeting all lines $L_{1}, L_{2}, L_{3}$ either $N \cap F_{1}=L_{1}$ or $N \cap F_{1}=J$ or $N \cap F_{1} \cap S$ contains a point of $L_{1} \cap S$ and a point of $L_{h} \cap J \cap S$ for some $h=2,3$ (say $h=2$ ). By the definition of $N$ given in Remark 11 in the latter case we have $o_{2}=L_{2} \cap J \cap S$, while the second case cannot occur. We exclude the case $N \cap F_{1}=L_{1}$, because $N \cap L_{1} \cap S=\left\{o_{1}\right\}$. We get $N \cap F_{1}=\left\langle\left\{o_{1}, o_{2}\right\}\right\rangle$. Let $H$ be a hyperplane containing $\left\{o, o_{1}, o_{2}, o_{3}\right\}$ (i.e. containing $\left.\left(N \cap F_{1}\right) \cup\left\{o_{2}, o_{3}\right\}\right)$. The scheme $\operatorname{Res}_{H}(Z)$ satisfies the Segre conditions in degree $d-1$ with respect to $\mathbb{P}^{4}$, all hyperplanes (because $B_{3}=\emptyset$ ), all planes (there is at most one, $N$, by Lemma and $w_{\operatorname{Res}_{H}(Z)}(N) \leq 2 d-3$ ) and with respect to $L_{1}, L_{2}, L_{3}, F_{1} \cap N$. Fix another $B \in B_{1}$. If $B=J$, then we use $o_{2}$. If $B \subset F_{1}$, then we use $N \cap F_{1}$ and Remark 3. In the other case since $S \subset F_{1} \cup L_{2} \cup L_{3}$, then $B \cap S=\left\{u_{1}, u_{2}, u_{3}\right\}$ with $u_{1} \in F_{1}, u_{2} \in L_{2}$ and $u_{3} \in L_{3}$.

Lemma 15. Assume that $N$ exists and that either $\mathcal{B}=\emptyset$ or the elements of $\mathcal{B}$ are not through a common point $q$ and hence $\sharp(\mathcal{B}) \geq 3$ and there is a plane $A$ containing all of them (Remark 13). Then $h^{1}\left(\mathcal{I}_{Z}(d)\right)=0$.

Proof. If $\mathcal{B}=\emptyset$, then we use Lemma 14. Assume $\mathcal{B} \neq \emptyset$ (and hence $\sharp(\mathcal{B}) \geq 3$ and the existence of $A$ ) and fix $R_{1} \in \mathcal{B}$, say $R_{1} \in \mathcal{B}_{1}$. We have $h^{0}\left(\mathcal{I}_{R_{1} \cup L_{1} \cup L_{2} \cup L_{3}}(2)\right) \geq 4$. Let $\eta$ be the base locus of the linear system

$$
\left|\mathcal{I}_{R_{1} \cup L_{1} \cup L_{2} \cup L_{3}}(2)\right| .
$$

Let $Q$ be a general element $\left|\mathcal{I}_{R_{1} \cup L_{1} \cup L_{2} \cup L_{2} \cup L_{3}}(2)\right|$. By Remarks 11 and 13 $\operatorname{Res}_{Q}(Z)$ satisfies the Segre condition in degree $d-2$ and so $h^{1}\left(\mathcal{I}_{Z}(d)\right)=0$, unless $S \subset \eta$. By Lemma 12 we have $R_{1} \nsubseteq N$ and hence $A \neq N$ and $N \cap R_{1}$ is at most one point $u_{1}$. If $N \cap R_{1}=\emptyset$, then set $m_{u_{1}}:=0$. Set 
$\left\{e_{1}\right\}:=R_{1} \cap\left(L_{1} \cup L_{2} \cup L_{3}\right)$. We get $3 d+2=w(N)+w(R \geq d-2+\alpha$, where $\alpha$ is the sum of the multiplicities among the points $u_{1}, e_{1}, o_{1}, o_{2}, o_{3}$ with the convention that if $N \cap R \neq \emptyset$ and $u_{1} \in\left\{e_{1}, o_{1}, o_{2}, o_{3}\right\}$, then $\alpha=m_{e_{1}}+m_{o_{1}}+m_{o_{2}}+m_{o_{3}}$. We first get that $N \cap R \neq \emptyset$, the points $u_{1}, e_{1}, o_{1}, o_{2}, o_{3}$ are distinct and then $m_{e_{1}}+m_{u_{1}}+m_{O_{1}}+m_{O_{2}}+m_{o_{3}} \geq 2 d+4$.

(a) Assume $\mathcal{B}=\left\{R_{1}, R_{2}, R_{3}\right\}$ with $R_{i} \cap L_{j} \neq \emptyset$ if and only if $i=j$. We get points $u_{h}$ and $e_{h}, h=2,3$, with $m_{e_{h}}+m_{u_{h}}+m_{o_{1}}+m_{o_{2}}+m_{o_{3}} \geq 2 d+4$. We have $h^{0}\left(\mathcal{I}_{A \cup L_{1} \cup L_{2} \cup L_{3}}(2)\right) \geq 3$. Since $\sharp\left(B \cap R_{i}\right) \geq 3$ for all $i$, we get $R_{i} \subset \eta$ for all $i$. Since $A$ contains the 3 distinct lines $R_{1}, R_{2}, R_{3}$, then $A \subset \eta$. If $A \neq F_{h}$ for any $h$, then Lemma 9 gives $h^{0}\left(\mathcal{I}_{A \cup L_{1} \cup L_{2} \cup L_{3}}(2)\right)=3$, a contradiction. Assume for instance $A \subset M:=\left\langle L_{1} \cup L_{2}\right\rangle$. The set $M \cup L_{3}$ is the base locus of $\left|\mathcal{I}_{M \cup L_{3}}(2)\right|$. Since $R_{1} \cup L_{1} \cup L_{2} \cup L_{3} \subset M \cup L_{3}$, we get $S \subset M \cup L_{3}$. Since $N \cap L_{3}$ is a single point, $o_{3}$ with $m_{O_{3}}<d$ (Lemma 3) and $m_{O_{3}}+d+1<w(N)$, we have $N \subset M$. By assumption $L_{1} \nsubseteq A$. Since $L_{1} \cap N=\left\{o_{1}\right\}, L_{1} \nsubseteq A \cup N$. Since $L_{1} \cup A \cup N \subset \tau$, we get $h^{0}\left(\mathcal{I}_{B \cup L_{1} \cup L_{2} \cup L_{3}}(2)\right)=h^{0}\left(\mathcal{I}_{M \cup L_{3}}(2)\right)=3$, a contradiction.

(b) Now assume that $\sharp(\mathcal{B}) \geq 3$ and that $A \supset L_{h}$ for some $h$, say $h=1$. We have $\mathcal{B}=\mathcal{B}_{1}$, because each line of $A$ meets $L_{1}$ and $L_{1} \cap L_{j}=\emptyset$ if $j=2,3$. Fix $B \in \mathcal{B}$. Since $N \nsupseteq L_{1}$, we have $A \neq N$. We get that $A=\left\langle B \cup L_{1}\right\rangle$ and that $N \cap A$ is a line $T$ containing $o_{1}$. Call $\beta$ the base locus of $\left|\mathcal{I}_{B \cup L_{1} \cup L_{2} \cup L_{3}}(2)\right|$. As in part (a) it is sufficient to do the case $S \subset \beta$. If $L_{2} \cap A=L_{3} \cap A=\emptyset$, then Lemma 6 gives $h^{0}\left(\mathcal{I}_{A \cup L_{2} \cup L_{3}}(2)\right)=3<h^{0}\left(\mathcal{I}_{R_{1} \cup L_{1} \cup L_{2} \cup L_{3}}(2)\right) \geq 4$, a contradiction. Hence either $A \cap L_{2} \neq \emptyset$ or $A \cap L_{3} \neq \emptyset$, say $A \cap L_{2} \neq \emptyset$. Hence $A \subset M:=\left\langle L_{1} \cup L_{2}\right\rangle$.

(b1) First assume $L_{3} \cap A \neq \emptyset$. Since $L_{2} \nsubseteq A$ and $L_{3} \nsubseteq A$ all configurations $\left(A, L_{2}, L_{3}\right)$ with $\left\langle A \cup L_{2}\right\rangle \cap L_{3}=\emptyset$ are projectively equivalent, then $h^{0}\left(\mathcal{I}_{A \cup L_{2} \cup L_{3}}(2)\right)=5$ and $A \cup L_{2} \cup L_{3}$ is the base locus of $\left|\mathcal{I}_{A \cup L_{2} \cup L_{3}}(2)\right|$ (use reducible quadrics containing $A$ and that $\left\langle A \cup L_{2}\right\rangle \cap\left\langle A \cup L_{3}\right\rangle=A$ ). Assume for the moment $A \cap N \cap S \nsubseteq\left\{o_{1}, o_{2}, o_{3}\right\}$. Fix $Q^{\prime} \in\left|\mathcal{I}_{A \cup L_{2} \cup L_{3}}(2)\right|$. Since $L_{1} \subset A$ and each element of $\mathcal{B}$ is contained in $A$, our assumption gives that $\operatorname{Res}_{Q^{\prime}}(Z)$ satisfies the Segre conditions in degree $d-2$. Hence $h^{1}\left(\mathcal{I}_{Z}(d)\right)>0$, unless $Q^{\prime} \cap S=S$ for all $Q^{\prime}$, i.e. unless $S \subset A \cup L_{2} \cup L_{3}$. Hence $N \cap S \subset A \cap N \cup\left\{o_{2}, o_{3}\right\}$. We get that $A \cap N$ is a line $m_{O_{2}}+m_{o_{3}}=d,(A \cap N) \cap\left\{o_{2}, o_{3}\right\}=\emptyset$ and that $A \cap N \in B_{1}$. Since $o_{2}$ and $o_{3}$ are the only points of $N \cap\left(L_{2} \cup L_{3}\right)$, we get that either $A \cap N=L_{1}$ (not true, because $L_{1} \cap N=\left\{o_{1}\right\}$ ) or $B \in \mathcal{B}_{1}$ (and we use Lemma 13). Now assume $A \cap N \cap S \subseteq\left\{o_{1}, o_{2}, o_{3}\right\}$. Since $N \cap B \cap S \neq \emptyset$, each $B \in \mathcal{B}_{1}$ contains $o_{1}$, contradicting part (a) of Remark 13 .

(b2) Now assume $L_{3} \cap A=\emptyset$. Set $G:=\left\langle A \cup L_{2}\right\rangle$ and $\{u\}:=L_{3} \cap G$. In this case all configurations $\left(A, L_{2}, L_{3}\right)$ with $L_{2} \cap L_{3}=\emptyset$ are projectively equivalent, $h^{0}\left(\mathcal{I}_{A \cup L_{2} \cup L_{3}}(2)\right)=4$ and $A \cup\left\langle\{u\} \cup L_{2}\right\rangle \cup L_{3}$ is the base locus of $\left|\mathcal{I}_{A \cup L_{2} \cup L_{3}}(2)\right|$. Since $h^{0}\left(\mathcal{I}_{B \cup L_{1} \cup L_{2} \cup L_{3}}(2)\right) \geq 4$ and $B \cup L_{1} \subset A$, we get $\beta=A \cup\left\langle\{u\} \cup L_{3}\right\rangle \cup L_{3}$. 
Hence each point of $N \cap S$ is contained in one of the linear spaces $A,\left\langle\{u\} \cup L_{2}\right\rangle$, and $L_{3}$. We have $o_{3}=N \cap L_{3}$. Since $m_{o_{3}}+d+1<w(N), N$ contains at least 3 non collinear points of $M$ and hence $N \subset M$. Since $\{u\}$ is the only point of $L_{3} \cap M$, we get $u=o_{3}$ and $N \cap S \subset(A \cap S) \cup\left(\left\langle\left\{o_{3}\right\} \cup L_{2}\right\rangle \cap S\right)$. Since $\sharp(N \cap S) \geq 5$ (Remark 11) and $N \cap S$ spans $N$, we get that either $A=N$ (excluded by Lemma 13) or $A=\left\langle\left\{o_{3}\right\} \cup L_{2}\right\rangle$ (excluded because $L_{1} \subset A$ and $L_{1} \cap L_{2}=\emptyset$ ).

Lemma 16. Assume that $N$ exists and that $\mathcal{B} \neq \emptyset$. Then $h^{1}\left(\mathcal{I}_{Z}(d)\right)=0$.

Proof. Fix $B \in \mathcal{B}$, say $B \in \mathcal{B}_{1}$. We have $h^{0}\left(\mathcal{I}_{B \cup L_{1} \cup L_{2} \cup L_{3}}(2)\right) \geq 4$. Let $\eta$ be the base locus of the linear system $\left|\mathcal{I}_{B \cup L_{1} \cup L_{2} \cup L_{3}}(2)\right|$. Let $Q$ be a general element $\left|\mathcal{I}_{B \cup L_{1} \cup L_{2} \cup L_{2} \cup L_{3}}(2)\right|$. By Remarks 11 and $13 \operatorname{Res}_{Q}(Z)$ satisfies the Segre condition in degree $d-2$ and so $h^{1}\left(\mathcal{I}_{Z}(d)\right)=0$, unless $S \subset \eta$. Hence we may assume $S \subset \eta$. Set $E:=\left\langle L_{1} \cup B\right\rangle$. Since $L_{h} \cap L_{1}=\emptyset, h=1$, 2 , each $\left\langle E \cup L_{h}\right\rangle$, is a hyperplane. Assume $\left\langle E \cup L_{2}\right\rangle=\left\langle E \cup L_{3}\right\rangle$. Since $\left\langle L_{2} \cup L_{3}\right\rangle$ is a hyperplane, we get $L_{1} \subset\left\langle E \cup L_{2}\right\rangle \subset\left\langle L_{2} \cup L_{3}\right\rangle$. Hence $w\left(\left\langle L_{2} \cup L_{3}\right\rangle\right) \geq 3 d+3$, a contradiction. Therefore there is an index $h \in\{2,3\}$, say $h=3$, such that $L_{3} \nsubseteq\left\langle E \cup L_{2}\right\rangle$. Taking reducible quadrics $\left\langle E \cup L_{2}\right\rangle \cup M$ with $M$ a hyperplane containing $L_{3}$ we get that the base locus of $\left|\mathcal{I}_{E \cup L_{2} \cup L_{3}}(2)\right|$ is contained in $\left\langle E \cup L_{2}\right\rangle \cup L_{3}$. Since $B \cup L_{1} \subset E$, we get $\tau \subset\left\langle E \cup L_{2}\right\rangle \cup L_{3}$. Hence $N \cap S \subseteq\left(N \cap\left\langle E \cup L_{2}\right\rangle \cap S\right) \cup\left\{o_{3}\right\}$. Since $m_{o_{3}} \leq d-1$ (Lemma 3), $w(N)=2 d+1$ and $w(L) \leq d+1$ for every line $L$, we get $N \subset\left\langle E \cup L_{2}\right\rangle$. We have $E \neq N$, because $N \cap L_{1}=\left\{o_{1}\right\}$. If $L_{2} \nsubseteq \not\left\langle E \cup L_{3}\right\rangle$, we also get $N \subseteq\left\langle E \cup L_{2}\right\rangle \cap\left\langle E \cup L_{3}\right\rangle$. Since $L_{1} \subset E$ and $\left\langle L_{1} \cup L_{2} \cup L_{3}\right\rangle=\mathbb{P}^{4}$, we first get $\left\langle E \cup L_{2}\right\rangle \neq\left\langle E \cup L_{3}\right\rangle$ and then $N \subseteq E$, a contradiction.

Lemma 17. If $\mathcal{B} \neq \emptyset$, then $h^{1}\left(\mathcal{I}_{Z}(d)\right)=0$.

Proof. By Lemma 16 we may assume that $N$ does not exists. Fix $B \in \mathcal{B}$, say $B \in \mathcal{B}_{1}$. We have $h^{0}\left(\mathcal{I}_{B \cup L_{1} \cup L_{2} \cup L_{3}}(2)\right) \geq 4$. Let $\eta$ be the base locus of the linear system $\left|\mathcal{I}_{B \cup L_{1} \cup L_{2} \cup L_{3}}(2)\right|$. Let $Q$ be a general element $\left|\mathcal{I}_{B \cup L_{1} \cup L_{2} \cup L_{2} \cup L_{3}}(2)\right|$. By Remarks 11 and $13 \operatorname{Res}_{Q}(Z)$ satisfies the Segre condition in degree $d-2$ and so $h^{1}\left(\mathcal{I}_{Z}(d)\right)=0$, unless $S \subset \eta$. Hence we may assume $S \subset \eta$.

(a) Assume the existence of a point $q \in S$ such that each $R \in \mathcal{B}$ contains $q$; if $\sharp(\mathcal{B})=1$ we also assume $q \notin J \cup L_{1} \cup L_{2} \cup L_{3}$; we may do this by Lemma 11, because $J \notin \mathcal{B}$. Let $\beta$ be the base locus of $\left|\mathcal{I}_{\{q\} \cup L_{1} \cup L_{2} \cup L_{3}}(2)\right|$. Take $Q^{\prime} \in\left|\mathcal{I}_{\{q\} \cup L_{1} \cup L_{2} \cup L_{3}}(2)\right|$. Since $N$ does not exists, $\operatorname{Res}_{Q^{\prime}}(Z)$ satisfies the Segre conditions in degree $d-2\left(\right.$ Remark 10). Hence $h^{1}\left(\mathcal{I}_{Z}(d)\right)=0$, unless $S \subset \beta$. If $q \in J \cup L_{1} \cup L_{2} \cup L_{3}$, then we use Lemma 11. If $q \notin F_{h}$ for all $h$, then 
$\beta=\{q\} \cup L_{1} \cup L_{2} \cup L_{3}$; hence $\sharp(R \cap S) \leq 2$ for all $R \in \mathcal{B}$, a contradiction. Now assume $q \in F_{h} \backslash\left(J \cup L_{1} \cup L_{2} \cup L_{3}\right)$ for some $h$, say $h=1$. Since $\mathcal{I}_{F_{1} \cup L_{2} \cup L_{3}}(2)$ is spanned (use reducible quadrics $\left\langle F_{1} \cup L_{2}\right\rangle \cup M_{3}$ and $\left\langle F_{1} \cup L_{3}\right\rangle \cup M_{2}$ with $M_{i}$ a hyperplane containing $L_{i}$ ), we get $\beta \subset F_{1} \cup L_{2} \cup L_{3}$. Hence for each $B \in \mathcal{B}$ we get $B \cap S=\left(B \cap F_{1} \cap S\right) \cup\left(B \cap L_{2} \cap S\right) \cup\left(B \cap L_{3} \cap S\right)$; assume for the moment $R \nsubseteq F_{1}$ and hence $\sharp\left(R \cap F_{1} \cap S\right) \leq 1$; since $\sharp\left(B \cap L_{2}\right)+\sharp\left(B \cap L_{3}\right) \leq 1$, we get $\sharp(R \cap S) \leq 2$, a contradiction. Thus each element of $\mathcal{B}$ is contained in $F_{1}$. Hence it meets $L_{1}$. By Remark 3 we get $\mathcal{B}=\mathcal{B}_{1}$.

Let $\Psi$ be the set of all $R \in B_{1}$ such that $R \neq J, R \cap L_{2} \neq \emptyset$ and $R \cap L_{3} \neq \emptyset$. By Remark 3 for each $R \in \Psi$ we have $R \cap L_{2} \in S$ and $R \cap L_{3} \in S$.

Claim : We have $\sharp(\Psi) \leq 2$.

Proof of the Claim: Assume $\sharp(\Psi) \geq 3$ and fix 3 distinct elements $R_{1}, R_{2}$, $R_{3} \in \Psi$. If $R, T \in \Psi$ with $R \neq T$ and $R \cap T \neq \emptyset$, then the point $R \cap T$ is contained in $L_{2} \cup L_{3}$, because the plane $\langle R \cup T\rangle$ cannot contain $L_{2} \cup L_{3}$. Since $w\left(\mathbb{P}^{4} \backslash\left(L_{1} \cup L_{2} \cup L_{3}\right)\right) \leq d-2$, we get $3 d+3=w\left(R_{1}\right)+w\left(R_{2}\right)+w\left(R_{3}\right) \geq$ $d-2+w\left(L_{2}\right)+w\left(L_{3}\right)$, a contradiction.

Since $\sharp(\Psi) \leq 2$ and each $R \in \Psi$ contains a point of $L_{2} \cap S$ and a point of $L_{3} \cap S$, we may find $u \in L_{2} \cap S$ and $v \in L_{3} \cap S$ such that each element of $\Psi$ meets $\{u, v\}$. Let $H$ be a hyperplane containing $L_{1} \cup\{u, v\}$. It is sufficient to prove that $\operatorname{Res}_{H}(Z)$ satisfies the Segre conditions in degree $d-1$. It satisfies it with respect to $\mathbb{P}^{4}$ and, since $B_{3}=\emptyset$, all hyperplanes. To check the condition for lines, it is sufficient to check it for all $R \in B_{1}$. This condition is satisfied by all $R \in \mathcal{B}$, because $\mathcal{B}=\mathcal{B}_{1}$. It is satisfied by $L_{1}, L_{2}, L_{3}$ and by all $R \in B_{1}$ meeting $L_{1}$. By the definition of $\Psi, u, v$ it is satisfied by all $R \in B_{1}$, with $R \notin\left\{L_{2}, L_{3}\right\}$ and $R \cap L_{1}=\emptyset$. Assume $B_{2} \neq \emptyset$, say $B_{2}=\{F\}$. Since $N$ does not exists, we have $\sharp\left(S \cap F \cap\left(L_{1} \cup L_{2} \cup L_{3} \cup J\right)\right) \geq 4$.

(a1) Assume for the moment $\sharp\left(S \cap F \cap\left(L_{1} \cup L_{2} \cup L_{3}\right)\right) \geq 4$. Hence $L_{j} \subset F$ for some $j$. If $j=1$, then $\sharp(F \cap H \cap S) \geq 2$. Assume $j \neq 1$, say $j=2$. Since $u \in F \cap H \cap S$, to check the Segre condition for $F$ we may assume $w(F)=2 d+1$ and $F \cap L_{1} \cap S=\emptyset$. Since $w(F)+w\left(L_{1}\right)>3 d+1$, we get $F \cap L_{1}=\emptyset$. Hence $F \cap F_{1}$ is a unique point, e. Since $\left\langle L_{2} \cup L_{3}\right\rangle \nsupseteq F, F \cap L_{3}$, is at most one point, $o$; set $m_{o}=0$ if $F \cap L_{3}=\emptyset$. Since $S \subset F_{1} \cup L_{2} \cup L_{3}$, we get $d=w(F)-w\left(L_{2}\right)=$ $m_{e}+m_{o}$. By Lemma 3 we get $o \in S, F \cap L_{3} \neq \emptyset, F \cap L_{3} \in S$ and $m_{o}+m_{e}=d$. If $e=v$, then $w_{\operatorname{Res}_{H}(Z)}(F) \leq 2 d-1$. Hence we may assume that we cannot take $v=e$. In particular (since $\sharp\left(B_{2}\right)=1$ ) we may assume $\sharp(\Psi)=2$, say $\Psi=\left\{R_{1}, R_{2}\right\}$. Since $o \notin L_{1} \cup L_{2} \cup L_{3}$ and $w\left(\mathbb{P}^{4} \backslash\left(L_{1} \cup L_{2} \cup L_{3}\right)\right) \leq d-2$, we get $d-2-m_{o} \geq w\left(R_{1}\right)+w\left(R_{2}\right)-w\left(L_{2} \cap\left(R_{1} \cup R_{2}\right)\right)-w\left(L_{3} \cap\left(R_{1} \cup R_{2}\right)\right)$. Since $\sharp\left(L_{2} \cap S\right) \geq 3$, we have $w\left(L_{2} \cap\left(R_{1} \cup R_{2}\right)\right) \leq d$. Since $e \notin\left(R_{1} \cup R_{2}\right)$, we get $w\left(L_{3} \cap\left(R_{1} \cup R_{2}\right)\right) \leq d+1-m_{e}$. Hence $d-2-m_{o} \geq 2 d+2-d-d-1+m_{e}$, 
contradicting the equality $m_{e}+m_{o}=d$.

(a2) Now assume $\sharp\left(S \cap F \cap\left(L_{1} \cup L_{2} \cup L_{3}\right)\right) \leq 3$. First assume $J \subset F$. Since $w(F)-w(J) \geq d-1>w\left(\mathbb{P}^{4} \backslash\left(L_{1} \cup L_{2} \cup L-3\right)\right)$ there is $i \in\{1,2,3\}$ and $o_{i} \in L_{i} \cap S \cap F$ with $o_{i} \in F_{i}$. We get $F=F_{i}$. By Remark 12 we have $F \cap L_{i} \in S$ for all $i$. Since $\sharp\left(S \cap F \cap\left(L_{1} \cup L_{2} \cup L_{3}\right)\right) \leq 3$, we get $\left.S \cap F \cap\left(J \cup L_{1} \cup L_{2} \cup L_{3}\right)\right)=S \cap J$ and hence $w(F) \leq d+1$, a contradiction. Now assume $J \nsubseteq F$. Since $J$ is the only line containing 3 points of $L_{1} \cup L_{2} \cup L_{3}$, we get that $\sharp\left(S \cap F \cap\left(L_{1} \cup L_{2} \cup L_{3}\right)\right)=3, F=\left\langle S \cap\left(L_{1} \cup L_{2} \cup L_{3}\right)\right\rangle$ and $F \cap J$ is a point of $S \cap J \backslash S \cap J \cap\left(L_{1} \cup L_{2} \cup L_{3}\right)$. Hence each $F \cap F_{i}$ is a line not containing the point $J \cap L_{i}$. We get that $F_{1} \cup F_{2} \cup F_{3}$ is contained in the base locus of $\left|\mathcal{I}_{F \cup L_{1} \cup L_{2} \cup L_{3}}(2)\right|$. Since $\left|\mathcal{I}_{J \cup L_{1} \cup L_{2} \cup L_{3}}(2)\right|=\left|\mathcal{I}_{L_{1} \cup L_{2} \cup L_{3}}(2)\right|$, $\sharp\left(F \cap S \cap\left(J \cup L_{1} \cup L_{2} \cup L_{3}\right)\right) \geq 4$ and $F \cap\left(J \cup L_{1} \cup L_{2} \cup L_{3}\right)$ is not formed by 4 collinear points, we have $h^{0}\left(\mathcal{I}_{F \cup L_{1} \cup L_{2} \cup L_{3}}(2)\right) \geq 4>h^{0}\left(\mathcal{I}_{F_{1} \cup F_{2} \cup F_{3}}(2)\right)$, a contradiction.

(b) Assume that there is no point $q$ contained in all $R \in \mathcal{B}$. By Remark 13 we have $\sharp(\mathcal{B}) \geq 3$ and there is a plane $A$ containing all elements of $\mathcal{B}$. Since $A$ contains 3 distinct elements of $B_{1}$ and $\sharp(R \cap S) \geq 3$ for all $R \in B_{1}$, we have $A \subset \tau$. Since $B \subset A$, we get $h^{0}\left(\mathcal{I}_{A \cup L_{1} \cup L_{2} \cup L_{3}}(2)\right)=h^{0}\left(\mathcal{I}_{B \cup L_{1} \cup L_{2} \cup L_{3}}(2)\right) \geq 4$. If $A \neq F_{i}$ for all $i$ and $L_{i} \nsubseteq A_{i}$ for all $i$, then we get $\sharp\left(L_{i} \cap A\right)=1$ for all $i$ and and hence $h^{0}\left(\mathcal{I}_{A \cup L_{1} \cup L_{2} \cup L_{3}}(2)\right)=3$ (Lemma 9). Since $B \cup L_{1} \subset A$, and $h^{0}\left(\mathcal{I}_{B \cup L_{1} \cup L_{2} \cup L_{3}}(2)\right) \geq 4$, we get a contradiction. Now assume $L_{j} \subset A$ for some $j$. Since $\mathcal{B}_{1} \neq \emptyset$, we get $j=1$ and $\mathcal{B}_{2}=\mathcal{B}_{3}=\emptyset$. If $A \cap L_{2}=A \cap L_{3}=\emptyset$, then $h^{0}\left(\mathcal{I}_{A \cup L_{2} \cup L_{3}}(2)\right)=3$ (Lemma 6 ) and again we get a contradiction. Hence we may assume $A \cap L_{2} \neq \emptyset$, i.e. $A \subset\left\langle L_{1} \cup L_{2}\right\rangle$.

(b1) First assume $L_{3} \cap A=\emptyset$, i.e. $A \neq F_{1}$. For a general $v \in A$ we have $h^{0}\left(\mathcal{I}_{\{v\} \cup L_{1} \cup L_{2} \cup L_{3}}(2)\right)=5$ and $\{v\} \cup J \cup L_{1} \cup L_{2} \cup L_{3}$ is the base locus of $\left|\mathcal{I}_{\{v\} \cup L_{1} \cup L_{2} \cup L_{3}}(2)\right|$ (Lemma 11). Hence $h^{0}\left(\mathcal{I}_{A \cup L_{2} \cup L_{3}}(2)\right) \leq 4$. We get that equality holds; write $w:=\left\langle A \cup L_{2}\right\rangle \cap L_{3}$ and $A^{\prime}:=\left\langle L_{2} \cup\{w\}\right\rangle$. The set $A \cup A^{\prime} \cup L_{3}$ is the base locus of $\left|\mathcal{I}_{A \cup L_{2} \cup L_{3}}(2)\right|$. Hence $S=(A \cap S) \cup\left(A^{\prime} \cap S\right) \cup\left(L_{3} \cap S\right)$ with $A \cup A^{\prime} \subset\left\langle L_{1} \cup L_{2}\right\rangle$. Take $u \in L_{2} \cap S$ and $v \in L_{3} \cap S$ and call $H$ a hyperplane containing $L_{1} \cup\{u, v\}$. It is sufficient to check that $\operatorname{Res}_{H}(Z)$ satisfies the Segre conditions in degree $d-1$ and this is done as in step (a), except for the planes. Hence we may assume $B_{2} \neq \emptyset$ and call $E$ the only element of $B_{2}$. Since $N$ does not exists, we get $\sharp\left(E \cap\left(L_{1} \cup L_{2} \cup L_{3}\right)\right) \geq 4$ and hence $E$ contains one of the lines $L_{i}$ (and exactly one such line). Hence $E \cap H \cap S \neq \emptyset$ for any choice of $u, v$ and so we may assume $w(E)=2 d+1$. If $L_{1} \subset E$, then $\sharp(E \cap H \cap S) \geq 3$ and hence $w_{\operatorname{Res}_{H}(Z)}(E) \leq 2 d-2$.

(b1.1) Now assume $L_{2} \subset E$. Since $L_{1} \cup\{u\} \subset H \cap S$, to prove the inequality $w_{\operatorname{Res}_{H}(Z)}(E) \leq 2 d-1$, we may assume $E \cap L_{1} \cap S=\emptyset$. Since 
$w(E)+w\left(L_{1}\right)>3 d+1$, the Segre conditions of $Z$ give $E \cap L_{1}=\emptyset$. Since $L_{2} \cap L_{1}=\emptyset$, we get $E \neq A$. Assume $E=A^{\prime}$. Since $A^{\prime} \subset\left\langle L_{1} \cup L_{2}\right\rangle$, we have $E \cap L_{1} \neq \emptyset$. Since $w(E)+w\left(L_{1}\right)>3 d+1$, we get $E \cap L_{1} \in S$ and hence $w_{\operatorname{Res}_{H}(Z)}(E) \leq 2 d-1$. Thus we may assume $E \neq A^{\prime}$. Since $L_{2} \cap L_{3}=\emptyset$, the set $E \cap L_{3}$ is either empty or a single point $e$; in the latter case we set $m_{e}=0$.

(b1.1.1) Assume $m_{e}=0$. Since $E \cap S$ spans $S$ (Remark 2), we get $E \subset\left\langle L_{1} \cup L_{2}\right\rangle$. Hence $E \cap L_{1} \neq \emptyset$, a contradiction.

(b1.1.2) Assume $m_{e}>0$. If $e \neq w$, then $\left\langle L_{1} \cup L_{2}\right\rangle \cap E=L_{2}$. Since $S \subset\left\langle L_{1} \cup L_{2}\right\rangle \cup L_{3}$, we get $S \cap E=\{e\} \cup\left(L_{2} \cap S\right)$ and hence $m_{e}=d$; we conclude by Lemma 3.

(b1.2) Now assume $L_{3} \subset E$. We get $w\left(E \backslash L_{3}\right)=d$. The set $D:=$ $E \cap\left\langle L_{1} \cup L_{2}\right\rangle$ is a line containing $w$ and $w(D \backslash\{w\})=d$. Since $A \cap L_{3}=\emptyset$, we have $e \notin A$ and hence $D \cap A$ is a single point, $\alpha$. If $D \nsubseteq A^{\prime}$ we get $D \cap A^{\prime}=\{w\}$ and hence $m_{\alpha}=d$; we conclude by Lemma 3. Now assume $D \subset A^{\prime}$. Call $\beta$ the only point of $D \cap L_{3}$. Fix $R \in \mathcal{B}_{1}$ and call $\gamma$ the only point of $R \cap L_{1}$. Since $w\left(\mathbb{P}^{4} \backslash\left(L_{1} \cup L_{2} \cup L_{3}\right)\right) \leq d-2, w(R)=d+1$ and $w(D \backslash\{w\})=d$, we get $d-2 \geq d+1-m_{\gamma}+d-m_{\beta}$ and hence $m_{\beta}+m_{\gamma} \geq d+3$, a contradiction.

(b2) Now assume $A=F_{1}$. Since $F_{1} \cup L_{2} \cup L_{3}$ is the base locus of $\left|\mathcal{I}_{F_{1} \cup L_{2} \cup L_{3}}(2)\right|$, we get $S \subset F_{1} \cup L_{2} \cup L_{3}$. We get a contradiction as in step (a).

\section{Proof of Lemma 1}

In this section we assume $B_{3}=\emptyset$ and conclude the proof of Lemma 1 .

(a) Assume the existence of $L, D \in B_{1}$ such that $L \cap D=\emptyset$. By section 6 and Remark 3 we may assume $S \cap R \cap(L \cup D) \neq \emptyset$ for any $R \in B_{1}$. Set $H:=\langle D \cup L\rangle$. By assumption $\operatorname{Res}_{H}(Z)$ satisfies the Segre conditions in degree $d-1$ with respect to lines. It satisfies it with respect to $\mathbb{P}^{4}$ (since $\sharp(H \cap S) \geq 4$ ) and with respect to the hyperplanes (since $B_{3}=\emptyset$ ). If $B_{2}=\emptyset$, we are done. Hence we may assume the existence of $N \in B_{2}$. By Lemma $7 N$ is unique. Since $w(L)+w(D)+w(N)>4 d+1$, we have $N \cap S \cap(L \cup D) \neq \emptyset$. Hence $h^{1}\left(\mathcal{I}_{Z}(d)\right)=0$, unless $w(N)=2 d+1$ and $\sharp(N \cap S \cap(L \cup D))=1$, say $N \cap S \cap L$ is a single point, $o$, and $N \cap S \cap D=\emptyset$. Let $\Delta$ be the set of all line $B \in B_{1}$ with $B \cap D=\emptyset$. Fix $q \in N \cap S \backslash\{o\}$ and set $H_{q}:=\langle\{o, q\} \cup D\rangle$. The scheme $\operatorname{Res}_{H_{q}}(Z)$ satisfies the Segre conditions in degree $d-1$ with respect to $\mathbb{P}^{4}$, all hyperplanes, $N$ and hence with respect to all planes since $\{N\}=B_{2}$, and with respect to all lines, except the elements of $\Delta$ not containing either $o$ or $q$. Hence $h^{1}\left(\mathcal{I}_{Z}(d)\right)=0$, unless $\sharp(\Delta) \geq 3$ and not all elements of $\Delta$ are through the same point. Since 
any two elements of $B_{1}$ disjoint from $D$ meet, we get the existence of a plane $A$ containing all elements of $\Delta$. Since $N \cap D=\emptyset$, we saw that each element of $\Delta$ meets $N \cap S$. Since we assumed that not all elements of $\Delta$ pass through the same point, we get that either $A=N$ (excluded, because $L \subset A$ ) or $A \cap N$ is a line. Note that each $R \in \Delta$ meets $(A \cap N) \cap S$ and that $\sharp(A \cap N \cap S) \geq 2$ by our assumptions on $\Delta$ (not all through a common point). Set $U:=\langle(A \cap N) \cup D\rangle$. The scheme $\operatorname{Res}_{U}(Z)$ satisfies the Segre conditions in degree $d-1$ with respect to $\mathbb{P}^{4}$, all hyperplanes, $N$ (because $\left.\sharp(A \cap N \cap S) \geq 2\right)$ and hence with respect to all planes since $\{N\}=B_{2}$, with respect to all elements of $\Delta$ (they contain of $A \cap N \cap S$ ) and of $B_{1} \backslash \Delta$ (they intersects $D \cap S$ ), concluding this case.

(b) Assume $B_{1} \neq \emptyset$ and that any two elements of $B_{1}$ meets. Fix $L \in B_{1}$. If there is $N \in B_{2}$ take two points of $p, q \in N \cap S$. Otherwise take any two elements of $p, q$ of $S \backslash\{p, q\}$. Let $H$ be a hyperplane containing $L \cup\{p, q\}$. The scheme $\operatorname{Res}_{H}(Z)$ satisfies the Segre conditions in degree $d-1$ with respect to $\mathbb{P}^{4}$ (since $\sharp(H \cap S) \geq 4$ ) all hyperplanes (since $B_{3}=\emptyset$ ), all planes (since either $B_{2}=\emptyset$ or the only element of $B_{2}$ has two points of $H \cap S$ ) and all lines (since each element of $B_{1}$ meets $L$ ).

(c) Assume $B_{3}=B_{1}=\emptyset$. Let $H$ be a hyperplane containing 4 points of $S$ with the only restriction that if $B_{2} \neq \emptyset$, say $B_{2}=\{N\}$, then $H$ contains two points of $N \cap S$. The scheme $\operatorname{Res}_{H}(Z)$ satisfies the Segre conditions in degree $d-1$ with respect to $\mathbb{P}^{4}$ (since $\sharp(H \cap S) \geq 4$ ) all hyperplanes (since $B_{3}=\emptyset$ ), all planes (since either $B_{2}=\emptyset$ or the only element of $B_{2}$ has two points of $H \cap S$ ) and all lines (since $B_{1}=\emptyset$ ).

Parts (a), (b) and (c) conclude the proof of Lemma 1.

\section{8. $d=4,5$}

Proposition 2. If $Z$ satisfies the Segre conditions in degree 4, then $h^{1}\left(\mathcal{I}_{Z}(4)\right)=0$.

Proof. By Lemma 3 we may assume $m_{1}=3$. Set $S_{i}:=\left\{p \in S: m_{p}=i\right\}$, $i=1,2,3$. Set $\{o\}:=S_{3}, h:=\sharp\left(S_{2}\right)$ and $c:=\sharp\left(S_{1}\right)$. Since $w\left(\mathbb{P}^{4}\right) \leq 17$, we have

$$
2 h+c \leq 14
$$

The elements of $B_{1}$ are the lines through $o$ containing a point of $S_{2}$ (there are $h$ of them, they meet only at $o$ and, since they have weight 5 , no 4 of them are in a plane and they are disjoint from $S_{1}$ ), the ones (call $B_{-}$the set of these lines) containing $o$ and two points of $S_{1}$ (they exists only if $c \geq 2$, they are disjoint 
from $S_{2}$ ), the ones (call $B_{+}$the set of these lines) formed by 5 collinear points of $S_{3}$ (they exists only if $c \geq 5$ and they are disjoint from $S_{2} \cup\{o\}$ ), the ones (call them $B_{=}$) containing 2 points of $S_{2}$ and one point of $S_{1}$, and the ones (call $B_{\vee}$ the set of these lines) containing a point of $S_{2}$ and 3 points of $S_{1}$. We call starred (resp. bistarred) a point of $S_{1} \cup S_{2}$ contained in a line $L \in B_{-}$(resp. $\left.L \in B_{\vee}\right)$.

We assume that Proposition 2 fails and we take a counterexample $S, Z$ with minimal weight. By [2] we may assume $h+c \geq 7$. Let $\ell: \mathbb{P}^{4} \backslash\{o\} \rightarrow \mathbb{P}^{3}$ be the linear projection from $o$. Set $S_{i}^{\prime}:=\ell\left(S_{i}\right), i=1,2$, and $S^{\prime}:=S_{1}^{\prime} \cup S_{2}^{\prime}$. We have $S_{1}^{\prime} \cap S_{i}^{\prime}=\emptyset$ and $\sharp\left(S^{\prime}\right)=h+c-\sharp\left(B_{-}\right)$. We say that a point of $S_{i}^{\prime}$ has multiplicity $i$. We say that $p \in S_{1}^{\prime}$ is a marked point if $\ell^{-1} \cap\left(S_{2} \cup S_{1}\right)$ is not a point, i.e. if it is the image of a line in $B_{-}$; in this case the fiber contains no element of $S_{2}$ and two elements of $S_{1}$.

Claim 1: If $h^{1}\left(\mathbb{P}^{3}, \mathcal{I}_{S^{\prime}}(2)\right)=0$, then $h^{1}\left(\mathcal{I}_{Z}(4)\right)=0$.

Proof of Claim 1: First assume $c>0$ and that not all points of $S_{1}^{\prime}$ are marked points. Fix $q^{\prime} \in S_{1}^{\prime}, q^{\prime}$ not marked, and call $q$ the point of $S_{1}$ with $\ell(q)=q^{\prime}$. Since $h^{1}\left(\mathbb{P}^{3}, \mathcal{I}_{S^{\prime}}(2)\right)=0$, there is quadric surface $Q^{\prime} \subset \mathbb{P}^{3}$ with $Q^{\prime} \cap S^{\prime}=S^{\prime} \backslash\{q\}$. Let $Q \subset \mathbb{P}^{4}$ be the quadric cone with vertex $o$ and $Q^{\prime}$ as a basis. We have $Q \cap S \neq S$ and $\operatorname{Res}_{Q}(Z) \subseteq\{o\} \cup S_{2} \cup\{q\}$. Hence it is sufficient to prove that $h^{1}\left(\mathcal{I}_{\{o\} \cup S_{2} \cup\{q\}}(2)\right)=0$. The Segre conditions for $Z$ gives that no plane through $o$ contains 4 points of $S_{2} \cup\{q\}$, that no plane contains 6 points of $S_{2} \cup\{q\}$ and that no line contains 3 points of $\{o\} \cup S_{2} \cup\{q\}$. Hence $h^{1}\left(\mathcal{I}_{\{o\} \cup S_{2} \cup\{q\}}(2)\right)=0$. Now assume $c>0$ (and hence $h \leq 6$ ), but that each point of $S_{1}^{\prime}$ is marked. Fix $q^{\prime \prime} \in S_{1}^{\prime}$ and call $q, q^{\prime}$ the point of $S_{1}$ with $q^{\prime \prime}$ as their image. Take a quadric surface $Q^{\prime} \subset \mathbb{P}^{3}$ with $Q^{\prime} \cap S^{\prime}=S^{\prime} \backslash\left\{q^{\prime \prime}\right\}$. Let $Q \subset \mathbb{P}^{4}$ be the quadric cone with vertex $o$ and $Q^{\prime}$ as a basis. We have $Q \cap S \neq S$ and $\operatorname{Res}_{Q}(Z) \subseteq\{o\} \cup S_{2} \cup\left\{q, q^{\prime}\right\}$. Hence it is sufficient to prove that $h^{1}\left(\mathcal{I}_{\{o\} \cup S_{2} \cup\left\{q, q^{\prime}\right\}}(2)\right)=0$. Since $q^{\prime \prime}$ is marked, there is a line $R$ with $R \cap S=$ $\left\{o, q, q^{\prime}\right\}$. The Segre conditions give that $\{o\} \cup S_{2} \cup\left\{q, q^{\prime}\right\}$ has no 4-secant line, $R$ is its unique 3 -secant line and that if $A$ is a plane containing at least 5 points of $\{o\} \cup S_{2} \cup\left\{q, q^{\prime}\right\}$, then either $A \supset R$ and $A$ contains $\left\{o, q, q^{\prime}\right\}$ and two points of $S_{2}$ or it contains 4 points of $S_{2}$ and only one among the points $q, q^{\prime}$. Hence $h^{1}\left(\mathcal{I}_{\{o\} \cup S_{2} \cup\left\{q, q^{\prime}\right\}}(2)\right)=0$. Now assume $c=0$. Fix $q \in S_{2}$ and set $E:=S_{2} \backslash\{q\}$. Taking a quadric surface $Q^{\prime}$ with $Q^{\prime} \cap S^{\prime}=S^{\prime} \backslash\{\ell(q)\}$ we reduce to prove that $h^{1}\left(\mathcal{I}_{\{o\} \cup E \cup 2 q}(2)\right)=0$. Let $\mu: \mathbb{P}^{4} \backslash\{q\} \rightarrow \mathbb{P}^{3}$ be the linear projection from $q$. Note that $\mu \mid S \backslash\{q\}$ is injective. Hence it is sufficient to prove that $h^{1}\left(\mathbb{P}^{3}, \mathcal{I}_{\mu(\{o\} \cup E)}(2)\right)=0$. We have $\sharp(\mu(\{o\} \cup E))=h \leq 7$. By the Segre condition no line contains 4 points of $\mu(\{o\} \cup E)$ and (since $w(U) \leq 13$ for every hyperplane $U)$ no plane contains $\geq 6$ points of $\mu(\{o\} \cup E)$. Hence 
$h^{1}\left(\mathbb{P}^{3}, \mathcal{I}_{\mu(\{o\} \cup E)}(2)\right)=0$.

(a) By Claim 1, to check the case $h=7$ it is sufficient to observe that in the case $c=0$ for each plane $A \subset \mathbb{P}^{3}$ we have $\sharp\left(A \cap S_{2}\right) \leq 5$ by the Segre conditions for $Z$. In a similar way we do the case $(h, c)=(6,1)$. Now assume $(h, c)=(6,2)$. If $S^{\prime}$ has a marked point, then $\sharp\left(S^{\prime}\right)=6$ and again $\sharp\left(A \cap S^{\prime}\right) \leq 5$ even for the planes $A \subset \mathbb{P}^{3}$ containing the marked point. Now assume that $S^{\prime}$ has no marked point. Assume the existence of a plane $A \subset \mathbb{P}^{3}$ such that $\sharp\left(A \cap S^{\prime}\right) \geq 6$. Let $H \subset \mathbb{P}^{4}$ be the hyperplane containing $o$ and with $\ell(H \backslash\{o\})=$ $A$. We have $\operatorname{Res}_{H}(Z)=\{o\} \cup S_{2}$ and $h^{1}\left(\mathcal{I}_{\operatorname{Res}_{H}(Z)}(3)\right)=0$, because $\{o\} \cup S_{2}$ has cardinality 8 , no 4 -secant line, and it is not in a plane.

(b) Assume $h=5$ and hence $c \leq 4$. Therefore $B_{+}=\emptyset$. The cases with $c \leq 2$ are done as in the case $h=6$. We assume $c=4$, because the proof for $c=3$ is very similar, but easier.

(b1) Assume $h^{1}\left(\mathcal{I}_{S}(2)\right)=1$. Since $h^{1}\left(\mathcal{I}_{\{o\} \cup S_{2}}(2)\right)=0$ (by the Segre condition for $Z$ ) we get the existence of a quadric $Q$ such that $\{o\} \cup S_{2} \subset Q$ and $2 \leq \sharp\left(Q \cap S_{3}\right) \leq 3$. The scheme $\operatorname{Res}_{Q}(Z)$ is contained in the scheme $W$ union of $2 o, S_{2}$ and 2 points of $S_{3}$. We have $h^{1}\left(\mathcal{I}_{Z}(4)\right) \leq h^{1}\left(\mathcal{I}_{W}(2)\right)$. Set $E:=W \backslash 2 o$. We have $h^{1}\left(\mathcal{I}_{W}(2)\right)=h^{1}\left(\mathbb{P}^{3}, \mathcal{I}_{\ell(E)}(2)\right)$. Assume the existence of a plane containing all points of $\ell(E)$. We get the existence of a hyperplane $U$ containing $o, S_{2}$ and 2 points of $S_{1}$. The scheme $\operatorname{Res}_{U}(Z)$ satisfies the Segre condition in degree 3 with respect to $\mathbb{P}^{4}$, because $\sharp(U \cap S) \geq 4$, and with respect to all lines, because $B_{+}=\emptyset$ and hence every element of $B_{1}$ meets $S_{2} \cup S_{3} \subseteq U \cap S$. Fix $B \in B_{2}$. Since $w(B) \geq 8, B$ contains at least 2 points of $U \cap S$ and hence $w_{\operatorname{Res}_{U}(Z)}(B) \leq 7$. Fix $B \in B_{3}$. Since $w(B) \geq 11, B$ contains at least 2 points of $U \cap S$ and hence $w_{\operatorname{Res}_{U}(Z)}(B) \leq 9$.

Hence $h^{1}\left(\mathcal{I}_{W}(2)\right)=0$, unless there a line containing 4 points of $\ell(E)$. If this line exists, then we get a plane $A$ with $w(A) \geq 3+2+2+1+1$ and hence $A \cap S$ contains exactly 2 points of $S_{1}, 2$ points of $S_{2}$ and $\{o\}$. Fix $p \in S_{2} \backslash S_{2} \cap A$ and set $H:=\langle\{p\} \cup A\rangle$. The scheme $\operatorname{Res}_{H}(Z)$ satisfies the Segre condition in degree 3 with respect to $\mathbb{P}^{4}$, because $\sharp(H \cap S) \geq 4$. It satisfies the Segre conditions with respect to all lines, because $S_{2} \cup S_{3} \subset H$ and $B_{+}=\emptyset$. Since $w\left(\mathbb{P}^{4} \backslash H\right) \leq 6$, each $B \in B_{3}$ contains at least 2 points of $H \cap S$ and hence to check the Segre condition for $U$ in degree 3 we may quote Lemma 4 . Fix $B \in B_{2}$. Since $w\left(\mathbb{P}^{4} \backslash H\right) \leq 6$, we have $B \cap H \cap S \neq \emptyset$ and hence to check the Segre in degree 3 we may assume $w(B)=9$; since $w\left(\mathbb{P}^{4} \backslash H\right) \leq 6$, we get $\sharp(B \cap H \cap S) \geq 2$, unless $w\left(\mathbb{P}^{4} \backslash H\right)=6$ (and hence $H \cap S=\{p\} \cup(A \cap S)$ ) and $\{o\}=A \cap B$. For each $q \in S_{3} \cap B$ set $H_{q}:=\langle\{q\} \cup A\rangle$. We get $h^{1}\left(\mathcal{I}_{\operatorname{Res}_{H_{q}}(Z)}(2)\right)=0$ and hence $h^{1}\left(\mathcal{I}_{Z}(2)\right)=0$, unless there is a plane $B_{q} \supset\{p\} \cup(B \cap S \backslash\{q\})$. If $B_{q}$ exists, then $B_{q} \cap B$ is a line containing $o$, at least another element of $S_{2}$ and 2 points 
of $S_{3}$, contradicting the Segre conditions for $Z$.

(b2) Assume $h^{1}\left(\mathcal{I}_{S}(2)\right)=0$. As in step (b1) we get the existence of a quadric $Q$ such that $S \backslash Q \cap S$ is a point of $S_{1}$. Working as in step (b1) we quickly get a contradiction.

(b3) Assume $h^{1}\left(\mathcal{I}_{S}(2)\right) \geq 2$.

(b3.1) Assume the existence of a line $L$ with $\sharp(S \cap L) \geq 4$. Since $B_{+}=\emptyset$, we get $\sharp(S \cap L)=4$ and that either $S_{1} \subset L$ or $L$ contains one point of $S_{2}$ and 3 points of $S_{1}$. In all case $L$ is spanned by 3 collinear points of $S_{1}$ and hence it is unique. Take $q \in L \cap S$ and set $T:=S \backslash\{q\}$. Let $H$ be a plane containing $L$ and with $a:=\sharp(A \cap H)$ maximal. By Lemma 3 we may assume $a \leq 8$.

If $a=6$, then $S \backslash S \cap L$ has no 3 -secant line and hence $h^{1}\left(H, \mathcal{I}_{H \cap S}(2)\right)=1$. The residual exact sequence of $H$ gives $h^{1}\left(\mathcal{I}_{S \backslash S \cap H}(1)\right)>0$ and hence the 4 points of $S \backslash S \cap H$ span a plan $A$. Since $a=6$, we have $A \cap L=\emptyset$. Since $\sharp(T)=9, T$ satisfies the Segre condition in degree 2. Fix a plane $E$. If $E \supset L$, then $\sharp(E \cap T)=-1+\sharp(E \cap S) \leq 4$. If $E \nsupseteq L$, we get that either $E=A$ and hence $\sharp(E \cap T)=3$ or $\sharp(T \cap A) \leq 5$, because $\sharp(S \cap A \cap E) \leq 2$. Fix a hyperplane $U$. If $U \supset L$, then we get $\sharp(U \cap T) \leq 5$ by the definition of $a$. Take $U \nsupseteq L$ and assume $\sharp(U \cap T) \geq 8$. Since $U$ contains at most one point of $L$ and all but one of the points of $S \backslash S \cap L$ have multiplicity $>1$, we get $w(U) \geq 8+6$, contradicting the Segre condition for $Z$. Hence $h^{1}\left(\mathcal{I}_{S \backslash\{q\}}(2)\right)=0$ and hence $h^{1}\left(\mathcal{I}_{S}(2)\right) \leq 1$, a contradiction.

Now assume $a=8$. Since $\sharp(S \backslash S \cap H)=2$, we have $h^{1}\left(\mathcal{I}_{S \backslash S \cap H}(1)\right)=0$. Hence $h^{1}\left(H, \mathcal{I}_{T \cap H}(2)\right)>0$. Since $\sharp(T \cap H)=9$ and $T$ has no 4 -secant line we get the existence of a plane $G \subset H$ with $\sharp(G \cap T) \geq 6$ and hence $\sharp(G \cap S) \geq 7$. Since $c=4$, we get $w(G) \geq 10$, a contradiction.

Now assume $a=7$. First assume $h^{1}\left(\mathcal{I}_{S \backslash S \cap H}(1)\right)=0$. As in the case $a=8$ we first get $h^{1}\left(H, \mathcal{I}_{T \cap H}(2)\right)=0$ and then a contradiction. Now assume $h^{1}\left(\mathcal{I}_{S \backslash S \cap H}(1)\right)>0$, i.e. assume the existence of a line $R \supset S \backslash S \cap H$. Taking $\langle L \cup R\rangle$ instead of $H$ we conclude, unless there is a line $D$ containing the 3 points of $S \cap H \backslash S \cap L$. Since $a=7$, we have $D \cap L=\emptyset=D \cap R$. Since $h^{1}\left(\mathcal{I}_{D \cup L \cup R}(2)\right)=0$, we get $h^{1}\left(\mathcal{I}_{S}(2)\right)=h^{1}\left(D \cup R \cup L, \mathcal{I}_{S}(2)\right)=1$, a contradiction.

(b3.2) Now assume the non-existence of a quadrisecant line of $S$. Let $E$ be a plane such that $b:=\sharp(E \cap S)$ is maximal. Let $M$ be a hyperplane with $a:=\sharp(M \cap S)$ maximal. By Lemma 3 we may assume $a \leq 8$.

First assume $b \geq 7$. Since $c=4$, we get $w(E) \geq 10$, a contradiction.

Now assume $b=6$. Since $w(E) \leq 9$, we get that $E$ contains 3 points of $S_{1}$ and 3 points of $S_{2}$ or 4 points of $S_{1}$ and two points of $S_{2} \cup S_{3}$. If $o \notin E$, let $H$ be a hyperplane containing $\{o\} \cup E$. If $o \in E$ let $H$ be a hyperplane 
containing $E$ and another point of $S_{2}$. Since $w\left(\mathbb{P}^{4} \backslash H\right) \leq 6, \operatorname{Res}_{H}(Z)$ satisfies the Segre conditions in degree 3 with respect to $\mathbb{P}^{4}$, all $U \in B_{3}$ and all $A \in B_{2}$ with $w(A)=8$. Take a plane $A$ with $w(A)=9$. We get $w_{W}(A) \leq 7$, unless $A \cap H \cap S=\{o\}$ and $w\left(\mathbb{P}^{4} \backslash H\right)=6$. In this case $E \cap S$ contains $\{o\} \cup S_{1}$ and exactly one point of $S_{2} S \backslash S \cap(E \cup A)$ is a point $p$ with multiplicity 2 and $A$ is spanned by $o$ and 4 points of $S_{2}$. Let $p^{\prime}$ be one of the points of $A \cap S_{2}$. Using $H^{\prime}:=\left\langle E \cup\left\{p^{\prime}\right\}\right\rangle$ instead of $H$ we get $h^{1}\left(\mathcal{I}_{\operatorname{Res}_{H^{\prime}}(Z)}(3)\right)=0$.

Now assume $b=5$. We get $h^{1}\left(A, \mathcal{I}_{S \cap A}(2)\right)=0$ for every plane $A$. If $a \leq 7$, then $h^{1}\left(M, \mathcal{I}_{S \cap M}(2)\right)=0$ by the Segre conditions; if $a=7$, we get $h^{1}\left(\mathcal{I}_{S}(2)\right) \leq 1$, because $h^{1}\left(\mathcal{I}_{S \backslash S \cap M}(1)\right) \leq 1$; if $a=6$ we get $h^{1}\left(\mathcal{I}_{S \backslash S \cap M}(1)\right) \leq 1$, because $S$ has no quadrisecant line. Assume $a=8$. Since $h^{1}\left(\mathcal{I}_{S \backslash S \cap M}(1)\right)=0$, it is sufficient to observe that for each $q \in S \cap M, S \cap M \backslash\{q\}$ satisfies the Segre conditions in degree 2 and hence $h^{1}\left(M, \mathcal{I}_{S \cap M}(2)\right) \leq 1+h^{1}\left(M, \mathcal{I}_{S \cap M \backslash\{q\}}(2)\right)=1$.

Now assume $b=4$. If $a \geq 6$, then we conclude as in the case $b=5$. Now assume $a=5$. We conclude, because the 5 points of $S \backslash S \cap M$ are not contained in a plane.

Now assume $b=3$, then $S$ has no trisecant line. If $a \geq 5$, we conclude as in the case $b=4,5$. If $a=4$, then $S$ is in linearly general position and hence the inequality $h^{1}\left(\mathcal{I}_{S}(2)\right) \leq \sharp(S)-9=1$ is classical.

(c) Assume $h=4$. We have $c \leq 6$. By [2] we may assume $c \geq 4$ (anyway, in the case $c \leq 3$ we have $\left.h^{1}\left(\mathcal{I}_{S^{\prime}}(2)\right)=0\right)$.

(c1) Assume $B_{+}=B_{\vee}=\emptyset$. Let $H$ be a hyperplane containing $o$ and at least 3 points of $S_{2}$. It is sufficient to prove that $\operatorname{Res}_{H}(Z)$ satisfies the Segre conditions in degree 3 . The condition for $\mathbb{P}^{4}$ is satisfied, because $\sharp(H \cap$ $S) \geq 4$. The condition for lines is satisfied, because $B_{+}=B_{\vee}=\emptyset, o \in H$ and $\sharp\left(S_{2} \backslash S_{2} \cap H\right) \leq 1$, so that $H \cap S$ meets each element of $B_{=}$. We have $w\left(\mathbb{P}^{4} \backslash H\right) \leq c+2 \leq 8$. Fix $U \in B_{x}, x=2,3$. We get $\sharp(S \cap U \cap H) \geq w(U)-c-3$. Hence each hyperplane satisfies the Segre condition for $\operatorname{Res}_{H}(Z)$. A plane $A$ may not satisfy the Segre condition for $\operatorname{Res}_{H}(Z)$ only if it contains 6 points of $S_{1}$ and either $o$ or a point of $S_{2} \backslash S_{2} \cap H$. Hence we may assume that $c=6$ and that $S_{1}$ is contained in a plane $A$. Since $w\left(S_{1}\right)=6$, we get $A=\left\langle S_{1}\right\rangle$. Therefore $A$ is unique. Hence if it contains a point $q \in S_{2}$, then we may take this point $q$ as a point of $S_{2} \cap H$, so that $A \cap S \cap H \neq \emptyset$; since $w(A)=8$, in this case $A$ satisfies the Segre condition for $\operatorname{Res}_{H}(Z)$. Now assume $o \in A$. Let $M$ be a hyperplane containing $A$ and at least one point of $S_{2}$. We have $\operatorname{Res}_{M}(Z)=2 o \cup E$ with $E \subset S_{2}$ and $\sharp(E) \leq 3$ and hence $h^{1}\left(\mathcal{I}_{2 o \cup E}(3)\right)=0$.

(c2) Assume $B_{+} \neq \emptyset$. Since $c \leq 6, B_{+}$has a unique element, $R$. Fix $p \in S_{2}$ and let $H$ be a hyperplane containing $\{o, p\} \cup R$. It is sufficient to prove that $\operatorname{Res}_{H}(Z)$ satisfies the Segre conditions in degree 3 . The condition for $\mathbb{P}^{4}$ 
is satisfied, because $\sharp(H \cap S) \geq 4$. Since $\mathbb{P}^{4} \backslash H$ contains at most $c-5 \leq 1$ points of $S_{1}$ and 3 points of $S_{2}$, the conditions for hyperplanes is satisfied. Fix $A \in B_{2}$. Since $w(A) \geq 8>w\left(\mathbb{P}^{4} \backslash H\right)$, we have $A \cap H \cap S \neq \emptyset$. Hence to check the Segre condition we may assume $w(A)=9$ and $\sharp(A \cap H \cap S)=1$. Since $c \leq 6$, we get $c=6$, that $A=\left\langle S_{1}\right\rangle$ and that $o \in A$. Let $M$ be a hyperplane containing $A$ and at least one point of $S_{2}$. We have $\operatorname{Res}_{M}(Z)=2 o \cup E$ with $E \subset S_{2}$ and $\sharp\left(S_{2}\right) \leq 3$ and hence $h^{1}\left(\mathcal{I}_{2 o \cup E}(3)\right)=0$. The Segre condition in degree 3 for $\operatorname{Res}_{H}(Z)$ is satisfied by lines $\notin B=$, because $R \subset H$ and $o \in H$. Since we may choose any $p \in S_{2}$ we may satisfies the Segre condition for lines, unless there are $D_{1}, D_{2} \in B_{=}$such that $S_{2} \cap D_{1} \cap D_{2}=\emptyset$. If $D_{1} \cap S_{1} \in R$ (resp. $D_{2} \cap S_{1} \in R$ ), it is sufficient to take $p \in S_{2} \cap D_{2}$ (resp. $p \in S_{2} \cap D_{1}$ ). Assume $R \cap D_{1}=R \cap D_{2}=\emptyset$. We get $c=6$ and that $D_{1} \cap D_{2}$ is the only point of $S_{1} \backslash S_{1} \cap R$. Fix $q \in R \cap S_{1}$ and let $H^{\prime}$ be a hyperplane containing $\left\langle D_{1} \cup D_{2}\right\rangle \cup\{q\}$. We have $\operatorname{Res}_{H^{\prime}}(Z)=3 o \cup\left(S_{1} \backslash\{q\}\right)$. Since $h^{1}\left(\mathcal{I}_{\ell\left(S_{1} \backslash\{q\}\right.}(2)\right)=0$, we have $h^{1}\left(\mathcal{I}_{3 o \cup\left(S_{1} \backslash\{q\}\right.}(3)\right)=0$ and hence $h^{1}\left(\mathcal{I}_{Z}(4)\right)=0$.

(c3) Assume $B_{+}=\emptyset$ and $B_{\vee} \neq \emptyset$. If all points of $S_{2}$ are bistarred, then, $c \leq 6$, there is a plane $B$ containing at least 3 points of $S_{2}$ and 5 points of $S_{1}$ and hence $w(B) \geq 11$, a contradiction. Hence there is a hyperplane $H$ containing $o$ and 3 points of $S_{2}$ and such that $H \cap S_{2}$ contains all bistarred points of $S_{2}$. The scheme $\operatorname{Res}_{H}(Z)$ satisfies the Segre conditions in degree 3 with respect to $\mathbb{P}^{4}$ (because $\sharp(H \cap S) \geq 4$ ) and with respect to all lines (because $B_{+}=\emptyset, o \in H$ and all bistarred points of $S_{2}$ are in $\left.H\right)$. Fix $U \in B_{3}$. Since $w\left(\mathbb{P}^{4} \backslash H\right) \leq 8$, we have $U \cap H \cap S \neq \emptyset$ and $\sharp(U \cap H \cap S) \geq 2$ if $w(U) \geq 12$. If $w(U)=13$ we may quote Lemma 4. Fix $A \in B_{2}$. Since $w\left(\mathbb{P}^{4} \backslash H\right) \leq 8$, we have $A \cap H \cap S=\emptyset$ only if $c=6, w(A)=8$ and $A$ contains $S_{1}$ and the point of $S_{2} \backslash S_{2} \cap H$; let $M$ be a hyperplane containing $A \cup\{o\}$; since $w\left(\mathbb{P}^{4} \backslash M\right) \leq 6, \operatorname{Res}_{M}(Z)$ satisfies the Segre conditions in degree 3 with respect to all linear spaces of dimension $>1$; it satisfies the condition for all lines, because $\{o\} \cup S_{1} \subset M$ and each element of $B_{1}$ meets $\{o\} \cup S_{1}$. Now assume the existence of a plane $A$ with $w(A)=9$ and $\sharp(A \cap H \cap S)=1$. Since $c \leq 6$, we get $c=6$ and $A \supset\{o\} \cup S_{1}$; take a hyperplane $M$ containing $A$ and an element of $S_{2}$ and proceed as above.

(d) Assume $h=3$. We have $c \leq 8$.

(d1) Assume $c=8$ and that $E:=\left\langle S_{1}\right\rangle$ is a plane. Let $M$ be a hyperplane containing $E \cup\{o\}$. It is sufficient to prove that $W:=\operatorname{Res}_{M}(Z)$ satisfies the Segre conditions in degree 3 . These conditions are satisfied by $\mathbb{P}^{4}$ (because $\sharp(M \cap S) \geq 4$ ) and by all lines (because $E \cup\{o\}$ meets each element of $B_{1}$ ). Fix $U \in B_{3}$. Since $S \backslash S \cap M \subseteq S_{2}$, we have $\sharp(U \cap M \cap S) \geq 3$. Fix $A \in B_{2}$. Since $S \backslash S \cap M \subseteq S_{2}$, we get $A \cap M \cap S \neq \emptyset$. Assume $w(A)=9$ and $\sharp(A \cap S)=1$. We get $S \cap H=S_{1} \cup\{o\}, A \cap S=\{o\} \cup S_{2}$ and hence that $A=\left\langle\{o\} \cup S_{2}\right\rangle$. We 
get $S \subset A \cup E$. Fix $q \in E \cap S$ and set $H_{q}:=\langle A \cup\{q\}\rangle$ and $W_{q}:=\operatorname{Res}_{H_{q}}(Z)$; if $B_{+} \neq \emptyset$, then assume that $q$ is one of the 5 points of the only elements of $B_{+}$. The last condition and the inclusion of $S_{2} \cup S_{3}$ in $H_{q}$ implies that $W_{q}$ satisfies the Segre condition in degree 3 with respect to all lines. It satisfies it with respect to $\mathbb{P}^{4}$ and with respect to all hyperplanes, because $W_{q}=2 o \cup S_{2} \cup\left(S_{1} \backslash\{q\}\right)$ and $w\left(\mathbb{P}^{4} \backslash H_{q}\right)=7$. Each $B \in B_{2}$ meets $H_{q} \cap S$. Assume $w(B)=9$ and $\sharp(B \cap S)=1$. We get that $\sharp\left(B \cap S_{1}\right) \geq 6$. Hence $B=\left\langle B \cap S_{1}\right\rangle$. Thus $B$ contains $q$ and at least one point of $S_{2} \cup S_{3}$, a contradiction.

(d2) Assume $B_{+}=\emptyset$; if $c=8$ assume that $\left\langle S_{1}\right\rangle$ is not a plane. Let $H$ be a hyperplane containing $S_{3} \cup S_{2}$ and spanned by points of $S$. We have $w\left(\mathbb{P}^{4} \backslash H\right) \leq 8$ with strict inequality if $\left\langle S_{3} \cup S_{2}\right\rangle$ is a plane. The scheme $\operatorname{Res}_{H}(Z)$ satisfies the Segre conditions in degree 3 with respect to $\mathbb{P}^{4}$ and all lines. Fix $U \in B_{3}$. We have $\sharp(U \cap H \cap S) \geq 2$, because $c=8$. If $w(U)=13$, then we use Lemma 4. Fix $A \in B_{2}$. Since $w\left(\mathbb{P}^{4} \backslash H\right) \leq 8$, we have $A \cap H \cap S=\emptyset$ if and only if $c=8$ and $A=\left\langle S_{1}\right\rangle$; we excluded this case. Now assume $w(A)=9$ and $\sharp(A \cap H \cap S)=1$. We get that either $A$ contains a point of $S_{2}$ and 7 points of $S_{1}$ or it contains $o$ and 6 points of $S_{1}$. Since $c \leq 8$ and we assumed $B_{+}=\emptyset$, there are at most two planes $A, A^{\prime}$, spanned by 6 points of $S_{1}$ and in this case we have $\sharp\left(A \cap A^{\prime} \cap S^{\prime}\right)=4, \sharp\left(A \cap S_{1}\right)=\sharp\left(S \cap A^{\prime}\right)=6$ and $A \cap A^{\prime}$ contains $o$ and 4 points of $S_{1}$, so that $w\left(A \cap A^{\prime}\right) \geq 7$, a contradiction. Hence we may assume that $A$ is the only plane spanned by 6 points of $S_{1}$. Let $M$ be a hyperplane containing $A$ and a point of $S_{3} \cup S_{2} \backslash\left(S_{3} \cup S_{2}\right) \cap A$ with maximal multiplicity. Since $w\left(\mathbb{P}^{4} \backslash M\right) \leq 6, \operatorname{Res}_{M}(Z)$ satisfies the Segre conditions in degree 3 with respect to all linear spaces of dimension $\geq 3$ and with respect to all planes with weight $\leq 8$. Fix a plane $B$ with $w(B)=9$ and assume $\sharp(B \cap M \cap S)=1$. It means that $B \cap M \cap S=\{o\}$ and that $B$ contains 2 points of $S_{2}$ not in in $M$ and 2 points of $S_{1}$. We get $w\left(\mathbb{P}^{4} \backslash H\right) \leq 6$ and in this case the plane $A$ does not arise.

(d3) Assume $B_{+} \neq \emptyset$; if $c=8$ assume that $\left\langle S_{1}\right\rangle$ is not a plane. Since $c \leq 8, B_{+}$has a unique element, $R$. Since $\sharp\left(S_{1} \backslash S_{1} \cap R\right)=3$ and $\left\langle S_{1}\right\rangle$ is not a plane, we get $\sharp\left(B_{\vee}\right) \leq 1$. Fix $q \in R \cap S$. Let $M$ be a hyperplane containing $q, o$ and two points of $S_{2}$; if $B_{\vee} \neq \emptyset$ we take inside $M$ at least one bistarred point of $S_{2}$. The scheme $\operatorname{Res}_{M}(Z)$ satisfies the Segre conditions in degree 3 with respect to $\mathbb{P}^{4}$. Now we check the Segre condition in degree 3 for lines for the scheme $\operatorname{Res}_{M}(W)$; since $\{q, o\} \in M$, it is satisfied, except at most by the elements of $B_{=}$and $B_{\vee}$. Since $\sharp\left(S_{2} \cap M\right)=2$, every element of $B_{=}$satisfies the Segre condition. By our choice of the points of $S_{2} \cap M$ every element of $B_{\vee}$ satisfies the Segre condition in degree 3.

Fix $U \in B_{3}$. Since $w\left(\mathbb{P}^{4} \backslash M\right) \leq 9$, we have $\sharp(M \cap U \cap S)>0$. Now assume 
$w(U)=12$ and $\sharp(M \cap U \cap S)=1$. Since $w\left(\mathbb{P}^{4} \backslash M\right) \leq 9$, we get $o \in U, c=8$ and that $U$ contains the point of $S_{2} \backslash S_{2} \cap M$ and the seven points of $S_{1} \backslash\{q\}$; since $R$ is spanned by two points of $R \cap S_{1}$, we get $q \in U$ and hence $\sharp(S \cap U \cap M) \geq 2$. If $w(U)=13$, then we use Lemma 4 .

Fix $B \in B_{2}$. If $B \cap M \cap S=\emptyset$, then $B$ must contain at least two points of $R \cap\left(S_{1} \backslash\{q\}\right)$, because $w\left(\mathbb{P}^{4} \backslash M\right) \leq 9$ and hence $q \in B$, a contradiction. Now assume that $B$ is a plane with $w(B)=9$ and $\sharp(B \cap M \cap S)=1$.

(d3.1) Assume $B \cap M \cap S \in S_{3} \cup S_{2}$. Since $q \notin B$, we get that $c=8$, $o \in B \cap M \cap S, B$ contains a point of $S_{2} \backslash S_{2} \cap M$, the 3 points of $S_{1} \backslash S_{1} \cap R$ and one point $p$ of $R \cap S_{1}$ with $p \neq q$. By the Segre condition for $Z$ we have $B=\left\langle\{o\} \cup\left(S_{1} \backslash S_{1} \cap R\right)\right\rangle$. Hence we may avoid the case taking inside $M$ the only point of $S_{2} \cap B$, because every $T \in B_{\vee}$ contains a point of $S_{1} \backslash S_{1} \cap R$.

(d3.2) Now assume $B \cap M \cap S \in S_{1}$. Hence $o \notin B$ and $\sharp\left(S_{2} \cap B\right) \leq 1$. Hence $\sharp\left(B \cap S_{1}\right) \geq 7$. Since $c \leq 8$, we get $\sharp\left(S_{2} \cap B\right)=1, R \subset B$ and $B=\left\langle B \cap S_{1}\right\rangle$. Since $c \leq 8, B$ is unique. Since $\sharp\left(S_{1} \backslash S_{1} \cap B\right)=1$, all elements of $B_{\vee}$ (if any) are contained in $B$. Hence we may take the point of $S_{2} \cap B$ as one of the points of $S_{2} \cap M$ and avoid this case (note that (d3.1) and (d3.2) cannot occur for different planes).

(f) Assume $h=2$. We have $c \leq 10$. Note that either $B_{=} \emptyset$ or $B=\left\{\left\langle S_{2}\right\rangle\right\}$.

(f1) Assume $\sharp\left(B_{+}\right) \geq 2$. Since $c \leq 10$, we have $\sharp\left(B_{+}\right)=2$, say $B_{+}=$ $\left\{D_{1}, D_{2}\right\}$ and either $D_{1} \cap D_{2}=\emptyset, c=10$ and $S_{1} \subset D_{1} \cup D_{2}$ or $D_{1} \cap D_{2} \neq \emptyset$ and the plane $\left\langle D_{1} \cup D_{2}\right\rangle$ contains at least 9 points of $S_{1}$ (by the Segre condition of $Z$ it contains 9 points of $S_{1}$ and no point of $S_{2} \cup S_{3}$ ). First assume $D_{1} \cap D_{2} \neq \emptyset$ and let $H$ be a hyperplane containing $D_{1} \cup D_{2} \cup\{o\}$. Since $w\left(\mathbb{P}^{4} \backslash H\right) \leq 5, \operatorname{Res}_{H}(Z)$ satisfies the Segre conditions in degree 3 with respect to all linear spaces of dimension $>1$. It satisfies the Segre condition for all lines, unless $c=10$, $\sharp\left(S_{1} \cap\left\langle D_{1} \cup D_{2}\right\rangle\right)=9$ and the line $\left\langle S_{2}\right\rangle$ contains the point of $S_{1} \backslash\left\langle D_{1} \cup D_{2}\right\rangle$. Set $F:=\left\langle D_{1} \cup S_{2}\right\rangle . F$ is a hyperplane. $\operatorname{Res}_{F}(Z)$ satisfies the Segre conditions in degree 3 with respect to $\mathbb{P}^{4}$ and all lines, because it contains $S_{2}$ and no line though $o$ may contain 2 points of $S_{1} \backslash S_{1} \cap F=D_{2} \cap S_{1} \backslash D_{1} \cap D_{2}$. Fix $U \in B_{3}$. Since $w\left(\mathbb{P}^{4} \backslash F\right) \leq 7$ and each point of $S \cap F$ has multiplicity $\leq 2$, we get $\sharp(F \cap U \cap S) \geq 2$, with strict inequality if $w(U)=13$. Fix $A \in B_{2}$. Since $w\left(\mathbb{P}^{4} \backslash F\right) \leq 7$, we get $A \cap F \cap S \neq \emptyset$. Now assume $w(A)=9$ and $\sharp(A \cap F \cap S)=1$. We get that $A \cap F \cap S \in S_{2}$ and that $A$ contains $o$ and the 4 elements of $S_{1} \backslash D_{1} \cap D_{2}$. Hence $D_{1} \cap D_{2} \in A \cap F \cap S$ and so $\sharp(A \cap F \cap S)>1$.

Now assume $D_{1} \cap D_{2}=\emptyset$. Fix $p_{i} \in D_{i} \cap S_{1}, i=1,2$, and let $G$ be a hyperplane containing $\left\{o, o_{2}, o_{3}\right\}$ and one point of $S_{2}$. By construction $\operatorname{Res}_{G}(Z)$ satisfies the Segre conditions with respect to $\mathbb{P}^{4}$ and all lines. Fix $U \in B_{3}$. Since $w\left(\mathbb{P}^{4} \backslash G\right) \leq 10$, we have $U \cap G \cap S \neq \emptyset$. Now assume $w(U)=12$ 
and $\sharp(U \cap G \cap S)=1$; since $w(U)-w\left(S_{2} \cup S_{3}\right) \geq 5$, $U$ must contain at least 4 points of $S \cap\left(D_{1} \cup D_{2}\right)$ and hence at least one of the points $p_{i}$; we also get that either $U \cap G \cap\left(S_{2} \cup S_{3}\right) \neq \emptyset$ or that $G \supset\left\{p_{1}, p_{2}\right\}$ and hence $\sharp(U \cap G \cap S)>1$. Now assume $w(U)=13$ and $\sharp(U \cap G \cap S)=2$; we exclude both the cases $\sharp\left(U \cap G \cap\left(S_{2} \cup S_{3}\right)\right)=2$ and $\sharp\left(U \cap D_{i} \cap S\right) \leq 1$ for all $i$ and the case $\sharp\left(U \cap G \cap\left(S_{2} \cup S_{3}\right)\right)=1$ and $\sharp\left(U \cap D_{i} \cap S\right)=1$ for at least one $i$ (because $3+5+1<13)$ and the case $\left.U \cap G \cap\left(S_{2} \cup S_{3}\right)\right)=\emptyset$ (because $c=10, o \notin U$ and $U$ has at most one element of $\left.S_{2}\right)$. Fix $A \in B_{2}$. Since $w\left(\mathbb{P}^{4} \backslash G\right) \leq 10$ and if $A$ contains 3 of the 8 points of $S_{1} \backslash\left\{o_{1}, o_{2}\right\}$, then it also intersects $\left\{o_{1}, o_{2}\right\}$, we get $A \cap G \cap S \neq \emptyset$. Now assume $w(A)=9$. If $A \cap G \cap\left(S_{3} \cup S_{2}\right)=\emptyset$, then $A \cap S$ is at most one point of multiplicity 2 and 7 points of multiplicity 1 and hence it contains $\left\{o_{1}, o_{2}\right\}$. If $\sharp\left(A \cap G \cap\left(S_{3} \cup S_{2}\right)\right)=1$, then it contains at least 6 points of $S \cap\left(D_{1} \cup D_{2}\right)$ and hence at least one of the points $o_{1}, o_{2}$.

(f2) Assume $\sharp\left(B_{+}\right)=1$, say $B_{+}=\{R\}$. Let $H$ be a hyperplane containing $S_{3} \cup S_{2}$ and $p \in R \cap S_{1}$. The scheme $\operatorname{Res}_{H}(Z)$ satisfies the Segre conditions in degree 3 with respect to $\mathbb{P}^{4}$, all lines and (since $w\left(\mathbb{P}^{4} \backslash H\right) \leq 9$ ) and all hyperplanes with weight $\leq 11$. By Lemma 4 we do not need to test the hyperplanes with $w(U)=13$. Let $U$ be a hyperplane with $w(U)=12$. Since $c \leq 10, U$ contains a point of $S_{2} \cup S_{3}$. If it contains a unique point of $S_{3} \cup S_{2}$, then it contains at least 9 points of $S_{1}$ and hence it contains two points of $R \cap S_{1}$ and hence $R$ and so another point $p$ of $H \cap S$. Take a plane $A$ with $w(A) \geq 8$ and assume $A \cap\left(S_{3} \cup S_{2}\right)=\emptyset$. Since $\left.c \leq 10, A \cap R \cap S_{1}\right) \geq 2$ and so $A \supset R$ and hence $A \cap H \cap S \neq \emptyset$. Now assume $w(A)=9, \sharp\left(A \cap\left(S_{3} \cup S_{2}\right)\right)=1$ and $p \notin A$. Since $c \leq 10$, we get $c=10, o \in A$, that $A$ contains all points of $S_{1} \backslash S_{1} \cap R$ and a unique point $q \in R \cap S_{1}$. If $A$ is unique, to avoid this case it is sufficient to take $q$ as the point $p$. Since $w(T) \leq 5$ for any line, $\left\langle\{o\} \cup\left(S_{1} \backslash S_{1} \cap R\right)\langle\right.$ is a plane and so $A=\left\langle\{o\} \cup\left(S_{1} \backslash S_{1} \cap R\right)\langle\right.$ is unique.

(f3) Assume $B_{+}=\emptyset$. Let $H$ be a hyperplane containing $S_{3} \cup S_{2}$ and $p \in$ $S_{1}$. The scheme $\operatorname{Res}_{H}(Z)$ satisfies the Segre conditions in degree 3 with respect to $\mathbb{P}^{4}$, all lines and (since $w\left(\mathbb{P}^{4} \backslash H\right) \leq 9$ ), all hyperplanes with weight $\leq 11$. Let $U$ be a hyperplane with $w(U)=12$. Since $c \leq 10$, we have $\left(S_{2} \cap S_{3}\right) \cap U \neq \emptyset$. If $\sharp\left(S_{2} \cup S_{3}\right) \geq 2$, then $U$ satisfies the Segre condition in degree 3 for $\operatorname{Res}_{H}(Z)$. If $\left(S_{2} \cap S_{3}\right) \cap U=\left\{o^{\prime}\right\}$ with $o^{\prime} \in S_{2} \cup S_{3}$, then either $o^{\prime} \in S_{2}, c=10$ and $U=\left\langle S_{1}\right\rangle$, or $o^{\prime}=o$ and $\sharp\left(U \cap S_{1}\right)=9$. In the first case $p \in U$ and hence $U$ satisfies the Segre condition in degree 3 for $\operatorname{Res}_{H}(Z)$. In the second case we may take $p \in U \cap S_{1}$ if $U$ is unique. If there is another $U^{\prime}$ containing $o$ and 9 points of $S_{1}$, we get that $U \cap U^{\prime}$ contain $o$ and at least 8 points of $S_{1}$ and so $w\left(U \cap U^{\prime}\right) \geq 11$ and so $U \cap U^{\prime}$ is not a plane, i.e. $U=U^{\prime}$. By Lemma 4 we do not need to test the hyperplanes with weight 13. 
Take a plane $A \in B_{2}$ with $\sharp(A \cap H \cap S) \leq 9-w(A)$.

(f3.1) First assume $A \cap\left(S_{3} \cup S_{2}\right)=\emptyset . A$ is the only plane containing at least 8 points of $S_{1}$; hence if $w(A)=8$ it is sufficient to take $p \in S_{1} \cap A$; note that in case all the hyperplanes $U, U_{1}$ contain $A$ and hence we may choose $p$ also for those hyperplanes. Thus we may assume $w(A)=9$. Set $M:=\langle\{o\} \cup A\rangle$. The scheme $\operatorname{Res}_{H}(Z)$ satisfies the Segre conditions in degree 3 with respect to $\mathbb{P}^{4}$ and (since $w\left(\mathbb{P}^{4} \backslash M\right) \leq 5$ and $m_{1}<4$ ) all linear spaces of dimension $>1$. It also satisfies the Segre conditions for lines, unless $c=10$ and the line $\left\langle S_{2}\right\rangle$ contains the point of $S_{1} \backslash S_{1} \cap A$; we assume that we are in this case. Let $M$ be a hyperplane containing $o$, one point of $S_{2}$ and two points $p_{1}, p_{2} \in A \cap S_{1}$. The scheme $\operatorname{Res}_{M}(Z)$ satisfies the Segre conditions in degree 3 with respect to $\mathbb{P}^{4}$ and all lines. Fix a hyperplane $U$ with $w(U) \geq 11$. By Lemma 4 we may assume $w(U) \leq 12$. If $U \cap M \cap\left(S_{3} \cup S_{2}\right)=\emptyset$, then $U$ contains at least 9 points of $S_{1}$ and hence at least 8 points of $S_{1} \cap A$; since these points span $A$ by the Segre condition for $Z$; we get $p_{1}, p_{2} \in U$. Now assume $\sharp\left(U \cap M \cap\left(S_{3} \cup S_{2}\right)\right)=1$; we get that $U$ has at least 5 points of $S_{1} \cap A$ and (since $B_{+}=\emptyset$ ), these points span $A$, so that $\sharp(U \cap M \cap S)>1$. Let $E$ be a plane with $w(E) \geq 8$ and $E \cap M \cap S=\emptyset$. We get that $\sharp\left(E \cap S_{1}\right) \geq 6$ and hence $\sharp\left(A \cap S_{1}\right) \geq 5$ and hence $E=A$ (since $B_{+}=\emptyset$ ) and hence $E \cap M \cap S \neq \emptyset$. Now assume $w(E)=9$ and $\sharp(A \cap M \cap S))=1$. As above we see that $\left.A \cap M \cap\left(S_{3} \cup S_{2}\right)\right)$ is a single point $o^{\prime}$ and that $E \cap S_{1}$ contains at least 3 point of $A$ (case $o^{\prime}=o$ ) or 4 points of $A$ (case $o^{\prime} \in S_{2}$ ); we may also assume $\left\langle E \cap A \cap S_{1}\right\rangle \neq A$, i.e. that all points of $A \cap S_{1} \cap E$ are collinear. In the case $o^{\prime} \in S_{2}$ there are at most 34 -secant lines of $A \cap S_{1}$ and all of them pass through 3 points of $A \cap S_{1}$; for suitable $p_{1}, p_{2}$ we find $E \cap M \cap S_{1} \neq \emptyset$ and hence $E$ satisfies the Segre condition in degree 3 for $\operatorname{Res}_{M}(Z)$. Now assume $o^{\prime}=o$. Since $E$ contains $S_{1} \backslash S_{1} \cap A$ and one point of $S_{2}$, it contains the other point of $S_{2}$, because $\left\langle S_{2}\right\rangle$ contains the point of $S_{1} \backslash S_{1} \cap A$.

(f3.2) Now assume that $A \cap\left(S_{3} \cup S_{2}\right)$ is a point $q$ with $m_{q}=2$. We get that $A$ contains at least 4 points of $A \cap S_{1}$. Since $A \neq E$, the points of $E \cap A \cap S_{1}$ are collinear. There are at most 34 -secant lines of $A \cap S_{1}$ and all of them pass through 3 points of $A \cap S_{1}$; for suitable $p_{1}, p_{2}$ we find $E \cap M \cap S_{1} \neq \emptyset$ and hence $E$ satisfies the Segre condition in degree 3 for $\operatorname{Res}_{M}(Z)$.

(f3.3) Now assume $\{o\}=A \cap\left(S_{3} \cup S_{2}\right)$. We get that $E$ contains at least 3 points of $A \cap S_{1}$. If $E$ contains 4 points of $A \cap S_{1}$, then we conclude as in step (f3.2). Thus we may assume that $E$ contains exactly 3 points of $A \cap S_{1}$ and hence it contains the point of $S_{1} \backslash S_{1} \cap A$. Since $E$ also contain a point of $S_{2}$ and the line $\left\langle S_{2}\right\rangle$ contains the point of $S_{1} \backslash S_{1} \cap A$, we get $S_{2} \subset E$ and hence $\sharp\left(E \cap A \cap\left(S_{3} \cup S_{2}\right)\right)=2$, a contradiction.

(g) Assume $h=1$. We have $c \leq 12$. Since $h=1, B_{-}=\emptyset$. Set 
$L:=\left\langle S_{3} \cup S_{2}\right\rangle$. Every $D \in B_{1} \backslash B_{+}$meets $L$.

(g1) Assume the existence of $L_{1}, L_{2} \in B_{+}$such that $L_{1} \cap L_{2}=\emptyset$. Since $L \cap L_{i} \cap S=\emptyset$, Remark 3 gives $L \cap L_{i}=\emptyset$. Since $\sharp\left(S_{1}\right)-\sharp\left(S_{1} \cap\left(L_{1} \cup L_{2}\right)\right) \leq 2$, we get $B_{+}=\left\{L_{1}, L_{2}\right\}$. Fix $p_{i} \in L_{i} \cap S, i=1,2$, and let $H$ be a hyperplane containing $L \cup\left\{p_{1}, p_{2}\right\}$. $\operatorname{Res}_{H}(Z)$ satisfies the Segre condition in degree 3 with respect to $\mathbb{P}^{4}$ and all lines. Fix $U \in B_{3}$. Since $w\left(\mathbb{P}^{4} \backslash H\right) \leq 10$, we have $U \cap H \cap S \neq \emptyset$. Assume $w(U)=12$. Since $c \leq 12$ and $S_{2} \cup S_{3} \subset H$, we get $S_{1}=U \cap S$ and hence $\left\{p_{1}, p_{2}\right\} \in U$. Lemma 4 takes care of the case $w(U)=13$. Fix $A \in B_{2}$. We have $A \cap S \cap H \neq \emptyset$, because $A \cap S$ cannot be the $c-10$ points of $S_{1} \backslash S_{1} \cap\left(L_{1} \cup L_{2}\right)$ and one point for each $L_{i}$. Now assume $w(A)=9$ and $\sharp(A \cap H \cap S)=1 ; A \cap H \cap S$ cannot be the $c-10$ points of $S_{1} \backslash S_{1} \cap\left(L_{1} \cup L_{2}\right)$, at most 5 points of $L_{i} \cap S_{1}$ and one point f $L_{3-i} \cap S_{1} ; A \cap H \cap S$ cannot be a point of $S_{3} \cup S_{2}$, the $c-10$ points of $S_{1} \backslash S_{1} \cap\left(L_{1} \cup L_{2}\right)$ and one point for each $L_{i}$.

(g2) Assume $\sharp\left(B_{+}\right) \geq 2$ and take $D_{1}, D_{2} \in B_{+}$with $D_{1} \neq D_{2}$. By step (g1) we may assume $D_{1} \cap D_{2} \neq \emptyset$. Hence $D_{1} \cap D_{2} \in S_{1}$, the plane $\left\langle D_{1} \cup D_{2}\right\rangle$ contains 9 points of $S_{1}$ and no other point of $S$. First assume that either $c \leq 11$ or the 3 points of $S_{1} \backslash S_{1} \cap\left\langle D_{1} \cup D_{2}\right\rangle$ are not contained in an element of $B_{\vee}$. Let $H$ be a hyperplane containing $D_{1} \cup D_{2} \cup\{o\}$. Our assumption gives that $\operatorname{Res}_{H}(Z)$ satisfies the Segre conditions in degree 3 with respect to $\mathbb{P}^{4}$ and all lines. Since $w\left(\mathbb{P}^{4} \backslash H\right) \leq 5, m_{1}<4$ and $m_{1}+m_{2}+5<13$, $\operatorname{Res}_{H}(Z)$ satisfies the Segre conditions in degree 3 with respect to all linear spaces of dimension $>1$. Now assume $c=12$ and the existence of $R \in B_{\vee}$ containing the 3 points of $S_{1} \backslash S_{1} \cap\left\langle D_{1} \cup D_{2}\right\rangle$. Let $M$ be a hyperplane containing $L \cup D_{1}$. Assume for the moment $B_{-} \emptyset$ and take $T \in B_{-}$; since $S_{1} \subset\left\langle D_{1} \cup D_{2}\right\rangle \cup R$, and $o \notin\left\langle D_{1} \cup D_{2}\right\rangle$, we get $T \cap R \neq \emptyset$ and hence $T \cap R \cap S \neq \emptyset$ (Remark 3 ). Thus $\operatorname{Res}_{M}(Z)$ satisfies the Segre conditions in degree 3 with respect to $\mathbb{P}^{4}$ and all lines. Fix $U \in B_{3}$. Since $w\left(\mathbb{P}^{4} \backslash M\right) \leq 7, \operatorname{Res}_{M}(Z)$ satisfies the Segre conditions in degree 3 with respect to all hyperplanes (if $w(U)=13$ use that $m_{1}+m_{2}+w\left(\mathbb{P}^{4} \backslash M\right)<13$ ) and all planes with weight $\leq 8$. Fix a plane $A$ with $w(A)=9$. If $A$ contains at least two points of $D_{2} \cap S_{1}$, then it contains $D_{2} \cap D_{1} \in M \cap S_{1}$. Since $S \backslash S \cap M$ is the union of $o$ and 4 points of $D_{2}$, then $\sharp(A \cap M \cap S)>1$.

(g3) Assume $\sharp\left(B_{+}\right)=1$, say $B_{+}=\{R\}$. By [2] we may assume $c \geq 6$. Fix $p \in R \cap S_{1}, q \in S_{1} \backslash S_{1} \cap R$ and take a hyperplane $H \supset L \cup\{p, q\} \operatorname{Res}_{H}(Z)$ satisfies the Segre conditions in degree 3 with respect to $\mathbb{P}^{4}$ and all lines. Fix $U \in B_{3}$. Since $w\left(\mathbb{P}^{4} \backslash H\right) \leq 10$, we have $S \cap H \cap U \neq \emptyset$. Assume $w(U)=12$ and $\sharp(U \cap S \cap H)=1$. Either $U \supset R$ and $U \cap\left(S_{2} \cup S_{3}\right)=\emptyset$ (and hence $c=12$ and $\left.U=\left\langle S_{1}\right\rangle\right)$ or $U$ contains one element of $S_{2} \cup S_{3}, c-5$ elements $S_{1} \backslash S_{1} \cap R$ and one element of $R$ (this case give $w(U) \leq 11$ ). Assume $c=12$ and $U=\left\langle S_{1}\right\rangle$; we 
get $\sharp(H \cap U \cap S)=2$, because $p, q \in H \cap U \cap S$. If $w(U)=13$, then we quote Lemma 4.

Fix $A \in B_{2}$. First assume $A \cap H \cap S=\emptyset$. Since $c-\sharp\left(S_{1} \cap R\right) \leq 7$ and $q \in H \cap S_{1}$, we get that $A$ contains at least two points of $R \cap S_{1}$ and hence it contains $p$, a contradiction. Now assume $w(A)=9$ and $\sharp(A \cap H \cap S)=1$. First assume $A \cap H \cap S \in\{p, q\}$. We get that $A$ contains 8 other points of $S_{1}$; call $G$ a hyperplane containing $A \cup\{o\}$; since $w\left(\mathbb{P}^{4} \backslash G\right) \leq 4, \operatorname{Res}_{G}(Z)$ satisfies the Segre conditions in degree 3. Now assume $\left(S_{3} \cap S_{2}\right) \cap A \neq \emptyset$ and hence that $A$ contains 6 or 7 points of $S_{1}$ (6 if and only $o \in A$ ), but neither $p$ nor $q$. Since $p \notin A, A$ contains at most one point of $R$. We have $A=\left\langle A \cap S_{1}\right\rangle$ and hence to avoid this case would be sufficient to take $q \in A$ if $A$ is unique. Take another $A^{\prime}$ containing one point of $S_{3} \cup S_{2}$ and 6 or 7 points of $S_{1}$ (6 if and only if $o \in A)$, but with at most one point of $R \cap S_{1}$. We get $\sharp\left(A \cap A^{\prime} \cap S_{1}\right) \geq 3$ and hence $A \cap A^{\prime}$ is a line. Since $w\left(\left\langle A \cup A^{\prime}\right\rangle\right) \geq 18-w\left(A \cap A^{\prime}\right) \leq 13$, we get $w\left(\left\langle A \cup A^{\prime}\right\rangle\right)=13$ and we use Lemma 4 .

(g4) Assume $B_{+}=\emptyset$ and the existence of a plane $E$ such that $\sharp\left(E \cap S_{1}\right) \geq$ 7. Let $F$ be a hyperplane containing $L$ and two points $p, q$ of $S_{1} \cap E$ and spanned by $S$. The scheme $\operatorname{Res}_{F}(Z)$ satisfies the Segre conditions in degree 3 with respect to $\mathbb{P}^{4}$, all lines and, since $w\left(\mathbb{P}^{4} \backslash F\right) \leq 7$, all planes with weight $\leq 8$ and (since $m_{1}<4$ ) all hyperplanes with weight $\leq 12$. For the hyperplanes with weight 13 we quote Lemma 4 . Take a hyperplane $U$ with $w(U)=13$; if $U \supset L$, then it meets each element of $B_{1}$, because $B_{+}=\emptyset$; since $c<w(U)$, $U$ contains at least one point, $o^{\prime}$, of $S_{2} \cup S_{3}$; if $U \cap\left(S_{2} \cup S_{3}\right)=\left\{o^{\prime}\right\}$, then $\sharp\left(U \cap S_{1}\right) \geq 10$ and hence $\sharp\left(U \cap E \cap S_{1}\right) \geq 5$; since $B_{+}=\emptyset$, we get $U \supset E$ and hence $\sharp(U \cap H \cap S) \geq 3$. Take a plane $B$ with $\sharp(B \cap F \cap S)=1$. If $B \cap\left(S_{3} \cup S_{2}\right)=9$, then $B$ has 9 points of $S_{1}$; since $B_{+}=\emptyset$, we get $B=E$ and hence $\sharp(B \cap F \cap S)>1$, a contradiction. Hence we may assume that $B$ contains one point of $S_{2} \cup S_{3}$ and at least 6 points of $S_{1}$, none of them on $R$. We have $B \cap E \cap S_{1} \neq \emptyset$. The planes $E, B$ are the only ones containing at least 6 points of $S_{1}$ and with weight 9 , the other one with at least 7 points of $S_{1}$ (because $B_{+}=\emptyset$ and hence $\left.\sharp\left(S_{1} \cap A \cap E\right) \leq 4\right)$. Hence we may take as $p$ a point of $B \cap S_{1}$.

(g5) Assume $B_{+}=\emptyset$ and the existence of a plane $A$ containing one point of $S_{3} \cup S_{2}$ and at least 6 points of $S_{1}$. Let $H$ be a hyperplane containing $A$ and the points of $\left(S_{3} \cup S_{2}\right) \backslash\left(S_{3} \cup S_{2}\right) \cap A$. The scheme $\operatorname{Res}_{H}(Z)$ satisfies the Segre conditions in degree 3 with respect to $\mathbb{P}^{4}$, all lines, all hyperplanes and all planes with weight $\leq 8$ (because $w\left(\mathbb{P}^{4} \backslash H\right) \leq 6$ and $m_{1}+m_{2}+6<13$ ). Fix a plane $B$ with $w(B)=9$ and $\sharp(A \cap B \cap S)=1$. We get that $B$ contains $o$ and 6 points of $S_{1} \backslash S_{1} \cap H$. First assume $o \in A$. We get $S_{2} \nsubseteq A \cup B$ and hence 
$Q \cap S \neq S$, where $Q$ is the union of a general hyperplane through $A$ and a general hyperplane through $B$. The inductive assumption gives $h^{1}\left(\mathcal{I}_{Z \cap Q}(4)\right)=0$. Since $o$ is a singular point of $Q$, we have $\operatorname{Res}_{Q}(Z)=\{o\} \cup 2 q$ (with $\{q\}=S_{2}$ ) and hence $h^{1}\left(\mathcal{I}_{\operatorname{Res}_{Q}(Z)}(2)\right)=0$. Now assume $o \notin A$. Since $o \in B$ we may reverse the role of $B$ and $A$ taking a hyperplane containing $B$ and $S_{2}$ and conclude as above.

(g6) Assume $B_{+}=\emptyset$. Let $H$ be a hyperplane containing $L$ and containing the maximal number $a \geq 2$ of points of $S_{1}$ among the hyperplanes containing $L$. The scheme $\operatorname{Res}_{H}(Z)$ satisfies the Segre conditions with respect to $\mathbb{P}^{4}$, all lines and (since $\left.w\left(\mathbb{P}^{4} \backslash H\right) \leq 10\right)$ all hyperplanes with weight $\leq 11$. Lemma 4 handles the hyperplanes with $w(U)=13$. Part (g4) handles the case of a plane $A$ with $w(A) \geq 8$ and $A \cap\left(S_{3} \cup S_{2}\right)=\emptyset$. Part (g5) handles the case of a plane $A$ with $w(A)=9$ and $\sharp\left(A \cap\left(S_{3} \cup S_{2}\right)\right)=1$. Fix a hyperplane $U$ with $w(U)=12$ and $\sharp(U \cap H \cap S)=1$. First assume $U \cap\left(S_{3} \cup S_{2}\right)=\emptyset$. We get $c=12$ and $U \supset S_{1}$; since $p, q \in H$, we get $\sharp(U \cap H \cap S)>1$. Now assume $U \cap\left(S_{3} \cup S_{2}\right) \neq \emptyset$. We get that $U$ contains 9 points of $S_{1}$ and $o$ or 10 points of $S_{1}$ and the point, $o^{\prime}$, of $S_{2}$. First assume $o^{\prime} \in S_{2} \cap U$; we get $a=2$ and in particular $B_{\vee}=B_{-}=\emptyset$; we get that $\operatorname{Res}_{U}(Z)$ satisfies the Segre conditions in degree 3 with respect to all lines and (since $w\left(\mathbb{P}^{4} \backslash U\right) \leq 5$ and $9-5>m_{1}$ ) with respect to all linear spaces of dimension $\geq 2$. Now assume $o \in U$; since $a \leq 3$, we get $B_{\vee}=\emptyset$; hence even in this case $\operatorname{Res}_{U}(Z)$ satisfies the Segre conditions in degree 3 with respect to all lines.

Proposition 3. If $Z$ satisfies the Segre conditions in degree $5, m_{1} \leq 3$ and $Z$ has $h$ points with multiplicity 3 with $h \in\{0,5,6,7\}$, then $h^{1}\left(\mathcal{I}_{Z}(5)\right)=0$.

Proof. Set $S_{i}:=\left\{p \in S: m_{p}=i\right\}$. Set $h:=\sharp\left(S_{3}\right), c:=\sharp\left(S_{2}\right)$ and $e:=\sharp\left(S_{1}\right)$. Since $w\left(\mathbb{P}^{4}\right) \leq 21$, we have

$$
3 h+2 c+e \leq 21
$$

By Lemma 1 we may assume $h \geq 2$. If $h=7$, then $\sharp(S)=7$ and this case is covered by [2]. For the same reason from now on we assume $h+c+e \geq 8$. For any $o \in S_{3}$ let $\ell_{o}: \mathbb{P}^{4} \backslash\{o\} \rightarrow \mathbb{P}^{3}$ be the linear projection from $o$. Set $S_{o}^{\prime}:=\ell_{o}(S \backslash\{o\})$. The Segre conditions for $Z$ gives $w(A) \leq 11$ for all planes $A$ and $w(U) \leq 16$ for all hyperplanes $U$. Every line spanned by two points of $S_{3}$ is in $S_{1}$ and it contains no other point of $S$.

Observation 1: Assume the existence of $o \in S_{3}$ such that $h^{1}\left(\mathbb{P}^{3}, \mathcal{I}_{S_{o}^{\prime}}(2)\right)=$ 0 . Fix $q \in S_{o}^{\prime}$. By assumption there is a quadric surface $Q^{\prime}$ such that $Q^{\prime} \cap S_{o}^{\prime}=$ $S_{o}^{\prime} \backslash\{q\}$. Let $Q=\{o\} \cup \ell^{-1}\left(Q^{\prime}\right)$ be the quadric cone with vertex containing 
$o$. Let $Z^{\prime \prime}$ be the union of the connected components of $Z$ whose support is a point of $S^{\prime} \backslash\{q\}$ whose image is $q$ and let $Z^{\prime}$ be the union of the connected components of $Z$ whose support is a point of $S^{\prime} \backslash\{q\}$ whose image is not $q$. Let $Z_{-}$be the closed subscheme of $Z^{\prime}$ obtained from $Z^{\prime}$ decreasing by one all the non-zero multiplicities of $Z^{\prime}$. We have $\operatorname{Res}_{Q}(Z) \subseteq\{o\} \cup Z_{-} \cup Z^{\prime \prime}$. Therefore to get $h^{1}\left(\mathcal{I}_{Z}(3)\right)=0$ it is sufficient to prove that $\{o\} \cup Z_{-} \cup Z^{\prime \prime}$ satisfies the Segre conditions in degree 3 .

(a) Assume $h=6$.

(a1) First assume $c=e=1$. Fix $o \in S_{3}$. The set $S_{o}^{\prime}$ has at most cardinality 7 . Therefore $h^{1}\left(\mathbb{P}^{3}, \mathcal{I}_{S_{o}^{\prime}}(2)\right)=0$, unless either it has 4 collinear points or it is contained in a plane. In the second case, $S$ is contained in a hyperplane and hence $h^{1}\left(\mathcal{I}_{Z}(5)\right)=0$. Assume that $S_{o}^{\prime}$ has a 4 -secant line. We get the existence of a plane $A \subset \mathbb{P}^{4}$ containing $o$ and at least 4 other points of $S$. Hence $w(A) \geq 12$, a contradiction.

Assume for the moment $\sharp\left(S_{o}^{\prime}\right)=7$. Let $q \in S_{o}^{\prime}$ be the image of the point with multiplicity 1 . The scheme $W:=\{o\} \cup Z_{-} \cup Z^{\prime \prime}$ has 5 points with multiplicity 2 (the points of $\left.S_{3} \backslash\{o\}\right)$ and 3 points with multiplicity one $\left(\{o\} \cup S_{2} \cup S_{1}\right)$. Since each line spanned by two points of $S_{3}$ contains no other point of $S,\{o\} \cup Z_{-} \cup Z^{\prime \prime}$ satisfies the condition for lines. We have $w_{W}\left(\mathbb{P}^{4}\right)=13$. Assume the existence of a line $L$ with $w_{W}(L) \geq 5$. Since $L$ must contain at least two points of $S_{3} \cup S_{2}$, we get $w(L) \geq 7$, a contradiction. Assume the existence of a plane $A$ such that $w(A) \geq 8$. Since $A$ must contain at least 4 points of $S_{3} \cup S_{2}$, we get $w(A) \geq 12$, a contradiction. Assume the existence of a hyperplane $U$ with $w(U) \geq 11$. Since $e=1, U$ contains either at least 6 points of $S_{3} \cup S_{2}$ or $o$ and at least 4 points of $\left(S_{3} \backslash\{o\}\right) \cup S_{2}$ and hence $w(U) \geq 15$, contradicting the Segre condition for $Z$.

Now assume $\sharp\left(S_{o}^{\prime}\right)<7$, i.e. assume the existence of a line $L$ containing $o$ and the two points of $S_{1} \cup S_{2}$, i.e. assume that $\left\langle S_{1} \cup S_{2}\right\rangle \in B_{1}$. To avoid this case it is sufficient to take as $o$ any of the five points of $S_{3}$ not in $\left\langle S_{1} \cup S_{2}\right\rangle$.

(a2) Now assume $e=2$. In this case $S$ has no 4 -secant line and no plane contains 7 points of $S$ (by the Segre conditions for $Z$ ) and $\sharp(S)=8$. Hence $h^{1}\left(\mathcal{I}_{S}(2)\right)=0$ and hence for each $q \in S_{1}$ there is a quadric hypersurface $Q$ with $Q \cap S=S \backslash\{q\}$. The inductive assumption gives $h^{1}\left(Q, \mathcal{I}_{Z \cap Q}(5)\right)=0$. We have $W:=\operatorname{Res}_{Q}(Z)=\sum_{p \in S_{3}} 2 p \cup\{u\}$ with $S_{1}=\{q, u\}$. We have $w_{W}\left(\mathbb{P}^{4}\right)=13$. Assume the existence of a line $L$ with $w_{W}(L) \geq 5$. Since it contains at least two points of $S_{3}$, we get $w(L) \geq 7$, a contradiction. Fix a hyperplane. By Lemma 3 we may assume $\sharp(S)-\sharp(S \cap U) \geq 2$ and hence $w_{W}(U) \leq 10$. Assume the existence of a plane $A$ with $w_{W}(A) \geq 8$. If $A$ contains at least 4 points of $S_{3}$, then we get $w(A) \geq 12$, a contradiction. Hence $S_{1} \subset A$ and $\sharp\left(A \cap S_{3}\right)=4$. Fix a hyperplane $H$ containing $A$ and another point of $S_{3}$. The scheme $\operatorname{Res}_{H}(Z)$ 
has $S_{3}$ as its support, one point of multiplicity 3 and 5 points of multiplicity 2 . It satisfies the Segre conditions in degree 3 , because no line contains 3 points of $S_{3}$, no plane contains 4 points of $S_{3}$ and no hyperplane contains $S_{3}$.

(b) Assume $h=5$ and hence $2 c+e \leq 6$ and $c+e \geq 3$.

(b1) Assume $c=3$ and hence $e=0$.

(b1.1) First assume that $\left\langle S_{2}\right\rangle$ is a line. Note that $S_{3} \cap\left\langle S_{2}\right\rangle=\emptyset$. Fix $o \in S_{3}$. By Observation 1 it is sufficient to prove that $h^{1}\left(\mathbb{P}^{3}, \mathcal{I}_{S_{o}^{\prime}}(2)\right)=0$. $S_{o}^{\prime}$ is gives by 4 points images of $\ell_{o}\left(S_{3} \backslash\{o\}\right)$ and 3 points on the line $\ell_{o}\left(\left\langle S_{2}\right\rangle\right)$. It is sufficient to prove that $S_{o}^{\prime}$ is contained in no plane (true, because $\langle S\rangle$ ) and no line contains 4 points of $S_{o}^{\prime}$. The latter condition is equivalent to the non-existence of a plane $E$ containing $\left\langle S_{2}\right\rangle, o$ and at least another point of $S_{3}$; this is true for any $o \in S_{3}$, because $w(E) \leq 11$.

(b1.2) Now assume that $\left\langle S_{2}\right\rangle$ is not a line. In this case every element of $B_{1}$ is spanned by two points of $S_{3}$. Let $H$ be a hyperplane containing 4 points of $S_{3}$. We check when $Y:=\operatorname{Res}_{H}(Z)$ satisfies the Segre conditions in degree 4 . First assume $S_{3} \subset H$. In this case the condition for lines is satisfied, because each element of $B_{1}$ meets $S_{3}$. Assume the existence of a hyperplane $U$ with $w_{Y}(U) \geq 14$. We have $U \neq H$, because $\sharp(H \cap S) \geq 4$. Since $w\left(\mathbb{P}^{4} \backslash H\right) \leq 6$, we get $\sharp\left(U \cap S_{3}\right) \geq 4$ and hence $w(U) \geq 8$, a contradiction. Fix a plane $A$ such that $w_{Y}(A) \geq 10$. We get $\sharp\left(A \cap S_{3}\right) \geq 2$ and hence $w(A) \geq 12$, a contradiction.

Now assume $S_{3} \nsubseteq H$, i.e. $S_{3}$ is linearly independent. Assume the existence of a plane $A$ such that $w_{Y}(A) \geq 10$. Since $w\left(S_{2}\right)=6$, we get $\sharp\left(S_{3} \cap A\right) \geq 2$. If $\sharp\left(A \cap S_{3}\right) \geq 3$, then $\sharp(S \cap A \cap H) \geq 2$ and hence $w(A) \geq 12$, a contradiction. Assume $\sharp\left(S_{3} \cap A\right)=2$. We get that $A$ contains $S_{2}$. Since $\left\langle S_{2}\right\rangle$ is not a line, we get $A=\left\langle S_{2}\right\rangle$ and it is sufficient to take inside $S_{3} \cap H$ both points of $S_{3} \cap\left\langle S_{3}\right\rangle$.

Assume the existence of a hyperplane $U$ with $w_{Y}(U) \geq 14$. We have $U \neq H$, because $\sharp(H \cap S) \geq 4$. Since $w\left(\mathbb{P}^{4} \backslash H\right) \leq 9$, we get $\sharp\left(S_{3} \cap U \cap H\right) \geq 2$ and hence $w(U) \geq 16$. By the Segre condition for $Z$ we have $w(U)=16$, i.e. $\sharp\left(S_{2} \cap U\right)=2$ and $\sharp\left(S_{3} \cap U\right)=4$. Hence $\sharp\left(U \cap H \cap S_{3}\right) \geq 3$ and so $w(U) \geq w_{Y}(U)+3=17$, a contradiction.

(b2) Assume $c=2$ and $e=2$. Fix $o \in S_{3}$. First assume $h^{1}\left(\mathbb{P}^{3}, \mathcal{I}_{S_{o}^{\prime}}(2)\right)=0$ and set $W:=\{o\} \cup Z_{-} \cup Z^{\prime \prime}$ with $q$ a point with multiplicity 1 . Therefore $W$ has 4 points with multiplicity 2 and 4 points with multiplicity 1 . It is sufficient to prove that $W$ satisfies the Segre conditions in degree 3 . We have $w_{W}\left(\mathbb{P}^{4}\right)=12$. By Lemma 3 we may assume $\sharp(S \cap U) \leq \sharp(S)-2$ for each hyperplane and hence $w_{W}(U) \leq 10$ for each hyperplane $U$. Assume the existence of a line $L$ with $w_{W}(L) \geq 5$. If $o \in L$, then $w(L) \geq 7$, a contradiction. If $o \notin L$, then $L$ contains at least two points of $S \backslash\{q\}$ and hence Now assume $h^{1}\left(\mathbb{P}^{3}, \mathcal{I}_{S_{o}^{\prime}}(2)\right)>0$. By Lemma 3 we may assume $\sharp\left(A \cap S_{o}^{\prime}\right) \leq 6$ for each plane $A$. Hence there is a line 
$L \subset \mathbb{P}^{3}$ with $\sharp\left(S_{o}^{\prime}\right) \geq 5$, i.e. there is a plane $A$ containing $o$ and 5 further points $S \backslash\{o\}$. We get $w(A) \geq 12$, a contradiction.

(b3) The cases $(c, e) \in\{(2,1),(1,2)\}$ are done as in step (b2). Assume $c=1$ and $e=4$. First assume that $L:=\left\langle S_{2} \cup S_{1}\right\rangle$ is a line. Fix $q, q^{\prime} \in S_{1}, q \neq q^{\prime}$. Since $\sharp\left(S_{3} \cup S_{2} \cup\left\{q, q^{\prime}\right\}\right)=8,\left\langle S_{3} \cup S_{2} \cup\left\{q, q^{\prime}\right\}\right\rangle=\left\langle L \cup S_{3}\right\rangle=\mathbb{P}^{4}$ and every secant line of $S_{3}$ does not contain other points of $S$, we have $h^{1}\left(\mathcal{I}_{S_{3} \cup S_{2} \cup\left\{q, q^{\prime}\right\}}(2)\right)=0$. Hence there is a quadric $Q$ with $Q \cap S=S \backslash\left\{p_{1}, p_{2}\right\}$ with $p_{1}, p_{2} \in S_{1}$ and hence it is sufficient to prove that $W:=\operatorname{Res}_{Q}(Z)$ satisfies the Segre conditions in degree $3 . W$ is the union of $S_{2},\left\{p_{1}, p_{2}\right\}$ and $\sum_{p \in S_{3}} 2 p$. We have $w_{W}\left(\mathbb{P}^{4}\right)=13$. Assume the existence of a line $R$ such that $w_{W}(R) \geq 5$. Since $R$ contains at least two points of $S_{3} \cup S_{2}$, we get $w(R) \geq 7$, a contradiction. Assume the existence of a plane $A$ with $w_{W}(A) \geq 8$. We get $\sharp\left(A \cap\left(S_{2} \cup S_{3}\right)\right) \geq 4$ and hence $w(A) \geq 12$, unless $A$ contains $\left\{p_{1}, p_{2}\right\}$. In this case $A$ contains $L$ and hence $w(A) \geq w_{W}(A)+3$, a contradiction. In the same way we get that if $U$ is a hyperplane with $w_{W}(U) \geq 11$, then $w(U) \geq 17$. Hence $\left\langle S_{2} \cup S_{1}\right\rangle$ is not a line, i.e. every element of $B_{1}$ meets $S_{3}$. Let $H$ be a hyperplane containing 4 points of $S_{3}$; if there is a line containing a point of $S_{3}$ and 3 points of $S_{1}$, then there is at most one such line and we impose that $H$ contains the point of $S_{3}$ in this line. We check when $Y:=\operatorname{Res}_{H}(Z)$ satisfies the Segre conditions in degree 4 . First assume $S_{3} \subset H$. In this case the condition for lines is satisfied, because each element of $B_{1}$ meets $S_{3}$. Assume the existence of a hyperplane $U$ with $w_{Y}(U) \geq 14$. We have $U \neq H$, because $\sharp(H \cap S) \geq 4$. Since $w\left(\mathbb{P}^{4} \backslash H\right) \leq 6$, we get $\sharp\left(U \cap S_{3}\right) \geq 4$ and hence $w(U) \geq 18$, a contradiction. Fix a plane $A$ such that $w_{Y}(A) \geq 10$. We get $\sharp\left(A \cap S_{3}\right) \geq 2$ and hence $w(A) \geq 12$, a contradiction.

Now assume $S_{3} \nsubseteq H$, i.e. $S_{3}$ is linearly independent. In this case the condition for lines is satisfied by our choice of $S_{3} \cap H$. Assume the existence of a plane $A$ such that $w_{Y}(A) \geq 10$. Since $w\left(S_{1} \cup S_{2}\right)=6$, we get $\sharp\left(S_{3} \cap A\right) \geq 2$. If $\sharp\left(A \cap S_{3}\right) \geq 3$, then $\sharp(S \cap A \cap H) \geq 2$ and hence $w(A) \geq 12$, a contradiction. Assume $\sharp\left(S_{3} \cap A\right)=2$. We get that $A$ contains $S_{2}$ and at least 3 points of $S_{1}$. Since $\left\langle S_{2} \cup S_{1}\right\rangle$ is not a line, there is at most one plane containing $S_{2}$ and at least 3 points of $S_{1}$ and it is sufficient to take inside $S_{3} \cap H$ both points of $S_{3}$.

Assume the existence of a hyperplane $U$ with $w_{Y}(U) \geq 14$. We have $U \neq H$, because $\sharp(H \cap S) \geq 4$. Since $w\left(\mathbb{P}^{4} \backslash\left(S_{1} \cup S_{2}\right)\right)=15$, we get $\sharp\left(S_{3} \cap U \cap H\right) \geq 2$ and $\sharp\left(S_{3} \cap U\right)=3$. We get $S_{2} \in U$ and that $U$ contains at least 3 points of $S_{1}$. Assume for the moment the existence of a plane $A \subset U$ containing $S_{2}$ and at least 3 points of $S_{1} ; A$ is spanned by $S_{2}$ and two points of $S_{1}$; we took $A$ containing 2 points of $S_{3} \cap H$; since $w(A) \leq 11$, we have $\sharp\left(S_{1} \cap A\right)=3$; we conclude taking $U$ instead of $H$, unless there is $B \in B_{1}$ with $B_{1} \cap U \cap S=\emptyset$; since $\left\langle S_{2} \cup S_{1}\right\rangle$ is not a line, the elements of $B_{1}$ are either the lines spanned by 
two points of $S_{3}$ or they contain 3 points of $S_{1}$ and in the latter case they are contained in $A$.

Now assume the non-existence of $A$; in this case $U$ is spanned by $S_{2} \cup\left(U \cap S_{1}\right)$ and it contains 3 points of $S_{3}$. We may take $U$ instead of $H$, because in this case no $A$ exists and every $B \in B_{1}$ mets a point of $U \cap S_{3}$.

In the same way we do the case $c=1$ and $e=3$.

(b4) Assume $c=0, e=6$ (the proof works if $c=0$ and $e<6$ ).

(b4.1) First assume the existence of a line $L$ with $\sharp\left(L \cap S_{1}\right)=6$, i.e. assume that $\left\langle S_{1}\right\rangle$ is a line. We claim that we may find $o \in S_{3}$ such that $\ell_{o}\left(S_{3} \backslash\{o\}\right) \cap \ell_{o}(L)=\emptyset$ and $h^{1}\left(\mathbb{P}^{3}, \mathcal{I}_{\ell_{o}\left(S_{3} \backslash\{o\}\right.}(2)\right)=0$. This is true, unless either a plane contains both $L \cup \ell_{o}\left(S_{3} \backslash\{o\}\right.$ ) (not possible, because $\left\langle S_{2}\right\rangle$ ) or a point of $\ell_{o}\left(S_{3} \backslash\{o\}\right)$ is contained in $\ell_{o}(L)$ (not possible, because it would give a plane $E$ containing $S_{1}, o$ and another point of $S_{3}$ and hence with $w(E)>11$ ) or $\ell_{o}\left(S_{3} \backslash\{o\}\right)$ is contained in a line (not possible, because no plane contains 4 points of $\left.S_{3}\right)$ or if $\ell_{o}\left(S_{3} \backslash\{o\}\right)$ contains 3 points in a line $D$ intersecting $\ell_{o}(L)$. In the latter case there is a hyperplane containing $\sharp(S)-1$ points of $S$ and hence $h^{1}\left(\mathcal{I}_{Z}(5)\right)=0$ by Lemma 3 . By the claim there is a quadric $Q$ containing $S_{3}$ and two points of $S_{1}$, with vertex containing $o$ and with $L \nsubseteq Q$, i.e. with $\sharp\left(Q \cap S_{1}\right)=4$. Since $Q \cap S \neq S$, the inductive assumption gives $h^{1}\left(\mathcal{I}_{Z \cap Q}(5)\right)=0$. The scheme $W:=\operatorname{Res}_{Q}(Z)$ is the union of $\{o\}, 4$ points of $S_{1} \cap L$ and the schemes $2 p, p \in S_{3} \backslash\{o\}$. It is sufficient to prove that it satisfies the Segre conditions in degree 3. We have $w_{W}\left(\mathbb{P}^{4}\right)=13$. $W$ satisfies the Segre conditions for lines, because $L \cap S_{3}=\emptyset$ and no line spanned by two points of $S_{3}$ meets $L$ (it would give a plane $E$ with $w(E)>11$. Fix $A \in B_{2}$. First assume $L \subset A$. We have $w(A \backslash L)=w(A)-6$ and hence $A$ contains at least $w(A)-10$ points of $S_{3}$; therefore $w_{W}(A) \leq 7$. Now assume $A \nsubseteq L$. Hence $A$ contains at most one point of $L \cap S_{1}=S_{1}$. If $S_{1} \cap A=\emptyset$, then $\sharp(A \cap S) \geq 4$ and hence $w_{W}(A) \leq 7$. Now assume $\sharp\left(S_{1} \cap A\right)=1$. If $w(A)=10$, then $\sharp\left(A \cap S_{3}\right) \geq 3$, while if $w(A)=11$ we have $\sharp\left(A \cap S_{3}\right)>3$, contradicting the inequality $w(A) \leq 11$. Fix $A \in B_{2}^{\prime}$, i.e. $8 \leq w(A) \leq 9$. If $A \supset L$, then we get $w_{W}(A) \leq w(A)-2 \leq 7$; if $A \nsupseteq L$ we first get $\sharp\left(S_{1} \cap A\right) \leq 1$, then $S_{3} \cap A \neq \emptyset$ and then, if $w(A)=9$, $\sharp\left(S_{3} \cap A\right) \geq 2$; in all cases we get $w_{W}(A) \leq 7$. Fix $U \in B_{3}$. First assume $U \supset L$. We have $w(U \backslash L)=w(U)-6$ and hence $\sharp\left(S_{3} \cap U\right) \geq 3$ with strict inequality if $w(U)=16$. Hence $w_{W}(U) \leq 10$. Now assume $U \nsupseteq L$ and hence $\sharp\left(U \cap A_{1}\right) \leq 1$. We get $\sharp\left(S_{3} \cap U\right) \geq 5$ and hence $S_{3} \subset U$; since $o$ appears with multiplicity one in $W$, we get $w(U) \leq 10$.

Fix $U \in B_{3}^{\prime}$, i.e. $11 \leq w(A) \leq 13$. Since $w(U)-w(L) \geq 7$, we always have $\sharp\left(S_{3} \cap U\right) \geq 3$ and hence $w_{W}(U) \leq 10$.

(b4.2) Now assume that $\left\langle S_{1}\right\rangle$ is not a line. Let $H$ be a hyperplane con- 
taining 4 points of $S_{3}$; there are at most two points of $S_{3}$ for which there are lines containing 3 points of $S_{1}$ and we take these points as some of the points of $S_{3} \cap H$. If $S_{3} \subset H$ we conclude as in step (b3). Thus we assume that $S_{3}$ is linearly independent. In this case the condition for lines is satisfied by our choice of $S_{3} \cap H$. Assume the existence of a plane $A$ such that $w_{Y}(A) \geq 10$. Since $w\left(S_{1}\right)=6$, we get $\sharp\left(S_{3} \cap A\right) \geq 2$. If $\sharp\left(A \cap S_{3}\right) \geq 3$, then $\sharp(S \cap A \cap H) \geq 2$ and hence $w(A) \geq 12$, a contradiction. Assume $\sharp\left(S_{3} \cap A\right)=2$. We get that $A$ contains at least 5 points of $S_{1}$. Since $\left\langle S_{1}\right\rangle$ is not a line, there is at most one plane containing at least 5 points of $S_{1}$ and it is sufficient to take inside $S_{3} \cap H$ both points of $S_{3}$.

Assume the existence of a hyperplane $U$ with $w_{Y}(U) \geq 14$. We have $U \neq H$, because $\sharp(H \cap S) \geq 4$. We have $\sharp\left(S_{3} \cap U \cap H\right) \geq 2$ and $\sharp\left(S_{3} \cap U\right)=3$. We get that $U$ contains at least 5 points of $S_{1}$. Assume for the moment the existence of a plane $A \subset U$ containing at least 5 points of $S_{1}$; we took $A$ containing 2 points of $S_{3} \cap H$; since $w(A) \leq 11$, we have $\sharp\left(S_{1} \cap A\right)=3$; we conclude taking $U$ instead of $H$, unless there is $B \in B_{1}$ with $B_{1} \cap U \cap S=\emptyset$; since $\left\langle S_{1}\right\rangle$ is not a line, the elements of $B_{1}$ are either the lines spanned by two points of $S_{3}$ or they contain 3 points of $S_{1}$ and in the latter case they are contained in $A \subset U$.

Now assume the non-existence of $A$; in this case $U$ is spanned by $U \cap S_{1}$ and it contains 3 points of $S_{3}$. We may take $U$ instead of $H$, because in this case no $A$ exists and every $B \in B_{1}$ mets a point of $U \cap S_{3}$.

\section{Acknowledgements}

The author was partially supported by MIUR and GNSAGA of INdAM (Italy).

\section{References}

[1] E. Ballico, On the Segre upper bound of the regularity for fat points in $\mathbb{P}^{4}$, I. Preprint.

[2] E. Ballico, O. Dumitrescu and E. Postinghel, On Segre's bound for fat points in $\mathbb{P}^{n}$. arXiv: 1504.05151 .

[3] B. Benedetti, G. Fatabbi and A. Lorenzini, Segre's bound and the case of $n+2$ fat points of $\mathbb{P}^{n}$. Comm. Algebra 40 (2012), 395-403.

[4] M. V. Catalisano, Linear systems of plane curves through fixed " fat " points of $\mathbf{P}^{2}$. J. Algebra 142 (1991), no. 1, 81-100. 
[5] Catalisano, M. V.; Trung, N. V.; Valla, G. A sharp bound for the regularity index of fat points in general position. Proc. Amer. Math. Soc. 118 (1993), no. $3,717-724$.

[6] G. Fatabbi, On the resolution of ideals of fat points. J. Algebra 242 (2001), no. $1,92-108$.

[7] G. Fatabbi and A. Lorenzini, On a sharp bound for the regularity index of any set of fat points. J. Pure Appl. Algebra 161 (2001), no. 1-2, 91-111.

[8] P. V. Thiên On Segre bound for the regularity index of fat points in $\mathbb{P}^{2}$. Acta Math. Vietnam. 24 (1999), no. 1, 75-81.

[9] P. V. Thiên, Segre bound for the regularity index of fat points in $\mathbb{P}^{3}$. J. Pure Appl. Algebra 151 (2000), no. 2, 197-214

[10] P. V. Thiên, Sharp upper bound for the regularity of zero-schemes of double points in $\mathbb{P}^{4}$. Comm. Algebra 30 (2002), no. 12, 5825-5847.

[11] N. V. Trung and G. Valla, Upper bounds for the regularity index of fat points. J. Algebra 176 (1995), no. 1, 182-209.

[12] N. C. Tu and T. M. Hung, On the regularity index of $n+3$ almost equimultiple fat points in $\mathbb{P}^{n}$. Kyushu J. Math. 67 (2013), no. 1, 20-213. 
